\title{
IL-1-driven stromal-neutrophil interactions define a subset of patients with inflammatory bowel disease that does not respond to therapies
}

\author{
Matthias Friedrich ${ }^{1,17}$, Mathilde Pohin (1) 1,17, Matthew A. Jackson ${ }^{1,17}$, Ilya Korsunsky ${ }^{2,3,4,5,6}$, \\ Samuel J. Bullers' ${ }^{1}$, Kevin Rue-Albrecht ${ }^{1}{ }^{1}$, Zoe Christoforidou', Dharshan Sathananthan ${ }^{8}$, \\ Tom Thomas ${ }^{8}$, Rahul Ravindran ${ }^{8}$, Ruchi Tandon ${ }^{8}$, Raphael Sanches Peres ${ }^{1}$, Hannah Sharpe ${ }^{9}$, \\ Kevin Wei ${ }^{6}$, Gerald F. M. Watts ${ }^{6}$, Elizabeth H. Mann ${ }^{10}{ }^{1}$, Alessandra Geremia ${ }^{8}$, Moustafa Attar ${ }^{1,10}$, \\ Oxford IBD Cohort Investigators ${ }^{\star}$, Roche Fibroblast Network Consortium, Sarah McCuaig ${ }^{\star}$, \\ Lloyd Thomas ${ }^{8}$, Elena Collantes ${ }^{8}$, Holm H. Uhlig ${ }^{\circledR 8,11}$, Stephen N. Sansom ${ }^{\circledR 1}$, Alistair Easton ${ }^{\circledR 1}{ }^{12}$, \\ Soumya Raychaudhuri $\mathbb{D}^{2,3,4,5,6,13}$, Simon P. Travis $\mathbb{1}^{8}$ and Fiona M. Powrie ${ }^{1}{ }^{\mathbb{A}}$
}

Current inflammatory bowel disease (IBD) therapies are ineffective in a high proportion of patients. Combining bulk and single-cell transcriptomics, quantitative histopathology and in situ localization across three cohorts of patients with IBD (total $n=376$ ), we identify coexpressed gene modules within the heterogeneous tissular inflammatory response in IBD that map to distinct histopathological and cellular features (pathotypes). One of these pathotypes is defined by high neutrophil infiltration, activation of fibroblasts and vascular remodeling at sites of deep ulceration. Activated fibroblasts in the ulcer bed display neutrophil-chemoattractant properties that are IL-1R, but not TNF, dependent. Pathotype-associated neutrophil and fibroblast signatures are increased in nonresponders to several therapies across four independent cohorts (total $n=343$ ). The identification of distinct, localized, tissular pathotypes will aid precision targeting of current therapeutics and provides a biological rationale for IL-1 signaling blockade in ulcerating disease.

nflammatory bowel diseases are a heterogeneous group of disorders characterized by inflammation throughout the gastrointestinal tract. The etiology involves maladaptation between the host and its intestinal microbiota, a dialog controlled by genetic and environmental factors ${ }^{1}$. The complex nature of IBD is reflected in its clinical phenotypes, encompassing both Crohn's disease (CD) and ulcerative colitis (UC) and a range of microscopic features such as granulomas, lymphoid aggregates, crypt abscesses and ulcers ${ }^{2,3}$. Treatments for IBD include general immunosuppressants (such as corticosteroids), immunomodulators (such as thiopurines) or biologics (such as antitumor necrosis factor- $\alpha$, TNF- $\alpha$ ) modulating specific inflammatory mediators ${ }^{4}$. However, identification of patients who will respond remains a major challenge.

We previously identified high expression of genes encoding the IL-6 family member oncostatin M (OSM), and its receptor OSMR, in the inflamed intestine of patients with IBD as being associated with nonresponse to anti-TNF therapy ${ }^{5}$. Notably, OSM produced by leukocytes signals primarily into stromal cells such as fibroblasts and endothelial cells. Subsequent transcriptomic studies have associated subsets of fibroblasts, inflammatory mononuclear phagocytes (MNPs), neutrophils and pathogenic T and plasma cells with therapy nonresponse in both $\mathrm{UC}$ and $\mathrm{CD}^{6-12}$. It remains unknown whether the cellular and molecular hallmarks of treatment response are uniform across patients or whether several different tissular pathologies are linked to therapy failure through distinct mechanisms. Further understanding in those areas is crucial to the design of personalized treatment regimens and new therapeutics for individuals that do not respond to current options.

Here, we explored how individual signatures of tissue inflammation associate with nonresponse to specific medical treatments. Transcriptomic changes linked to therapy nonresponse were found to reflect changes in cellular composition and activation state associated with distinct histological features. These molecular, cellular and histologic definitions of disease provide a basis for rational

'Kennedy Institute of Rheumatology, Nuffield Department of Orthopaedics, Rheumatology and Musculoskeletal Sciences, University of Oxford, Oxford, UK. ${ }^{2}$ Center for Data Sciences, Brigham and Women's Hospital, Boston, MA, USA. 'Division of Genetics, Department of Medicine, Brigham and Women's Hospital, Boston, MA, USA. ${ }^{4}$ Department of Biomedical Informatics, Harvard Medical School, Boston, MA, USA. ${ }^{5}$ Program in Medical and Population Genetics, Broad Institute of MIT and Harvard, Cambridge, MA, USA. ${ }^{6}$ Division of Rheumatology, Inflammation and Immunity, Department of Medicine, Brigham and Women's Hospital and Harvard Medical School, Boston, MA, USA. ${ }^{7}$ MRC Weatherall Institute of Molecular Medicine, Radcliffe Department of Medicine, University of Oxford, John Radcliffe Hospital, Oxford, UK. ${ }^{8}$ Translational Gastroenterology Unit, NIHR Oxford Biomedical Research Centre, Oxford University Hospitals NHS Foundation Trust, John Radcliffe Hospital, Oxford, UK. 9 Jenner Institute, Nuffield Department of Medicine, University of Oxford, Oxford, UK. ${ }^{10}$ The Wellcome Trust Centre for Human Genetics, University of Oxford, Oxford, UK. "Department of Paediatrics, John Radcliffe Hospital, Oxford, UK. ${ }^{12}$ Old Road Campus Research Building, Department of Oncology, Medical Sciences Division, University of Oxford, Oxford, UK. ${ }^{13}$ Centre for Genetics and Genomics Versus Arthritis, Manchester Academic Health Science Centre, University of Manchester, Manchester, UK. ${ }^{17}$ These authors contributed equally: Matthias Friedrich, Mathilde Pohin, Matthew A. Jackson. ${ }^{*}$ A list of authors and their affiliations appears at the end of

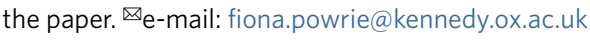


targeting of existing medications, and an alternative avenue to target inflammation in nonresponsive patients displaying ulceration with fibroblast and neutrophil remodeling.

\section{Results}

Identification of gene coexpression signatures in inflamed IBD tissue. Inflamed tissue from patients with IBD is commonly resected when either medical therapies have failed, when patients opt for elective surgery or when acute complications require emergency surgery. We used such tissues (referred to as difficult-to-treat IBD) as our discovery cohort (Extended Data Fig. 1). Amongst IBD tissue samples $(n=41)$ from the 31 patients in this cohort, 15 were macroscopically uninflamed, including seven samples for which paired uninflamed/inflamed tissue was available. We additionally profiled unaffected, nontumour tissue $(n=39)$ collected from 39 patients with colorectal cancer (CRC) and undergoing surgery as non-IBD controls. Bulk RNA-sequencing (RNA-seq) was used to generate whole-tissue gene expression profiles across all samples ( $n=41 \mathrm{IBD}$ and $n=39$ non-IBD; the discovery cohort').

To identify sets of genes reflective of distinct biological processes, we applied weighted gene correlation network analysis (WGCNA) to cluster coexpressed genes across all tissue samples. This identified 38 modules of highly coexpressed genes (M1-M38) (Supplementary Table 1). We correlated the expression (module eigengene) of these modules with sample characteristics, clinical phenotypes and histologic (microscopic) inflammation (Nancy index ${ }^{13}$ ); 28 modules were significantly associated with at least one of these measures (Fig. 1a). Modules were found to have dichotomous associations with traits: about half had significant positive correlations with histologic inflammation while the remainder had significant negative associations (Fig. 1a). Age appeared to have associations similar to inflammation, but this was an artifact of the older nature of the non-IBD samples used as controls. In a paired analysis of only inflamed and uninflamed IBD tissue samples from the same patients $(n=7)$, the difference in expression of a module between tissue pairs remained highly correlated with the module's association with histologic inflammation (Nancy index) $(R=0.8$, $P<0.001$; Extended Data Fig. 2a), confirming that these modules reflected inflammatory processes.

To determine whether the gene coexpression patterns detected reflect changes in the cell type composition of patient tissues, we applied in silico cell type deconvolution analysis to the RNA-seq data of our discovery cohort $\left(x \mathrm{Cell}^{14}\right)$. Correlating predicted cell type scores with module expression (eigengenes) (Extended Data Fig. 2b), modules positively correlated with histologic inflammation (Fig. 1a) were associated with signatures of stromal cells (for example, fibroblasts), B lymphocytes and plasma cells, Tlymphocytes (for example, $\mathrm{CD}^{+} \mathrm{T}$ cells) and granulocytes (for example, neutrophils). Modules negatively correlated with histologic inflammation were predicted to reflect epithelial and smooth muscle cells. These results suggest that the coexpression patterns associated with inflammation were, at least in part, driven by differences in cellular composition.

Coexpression signatures are associated with patient therapy response. To determine whether inflammation-associated modules are relevant to treatment outcomes, we projected the modules onto whole-tissue gene expression data derived from prospective studies of response to anti-TNF, corticosteroid or anti-integrin therapy ${ }^{10,15,16}$. At least $79 \%$ of the genes within each module could be identified in the three datasets, enabling accurate quantification within them (Extended Data Fig. 2c and Supplementary Table 2). The expression of 15 modules was significantly (adjusted $P<0.05$ ) higher or lower in nonresponders to anti-TNF before treatment (Supplementary Table 3). Seven and two modules, respectively, were significantly higher or lower in nonresponders to corticosteroid and anti-integrin therapy (Supplementary Table 3).
Strikingly, across all three therapy response datasets, modules M4 and M5 were consistently amongst the strongest associations with nonresponse in pretreatment samples (Supplementary Table 3 and Fig. 1b-d). This overall trend of increased expression in nonresponders was significant in meta-analyses of both M4 $(P=0.0025$, standardized mean difference $(S M D)=0.87,95 \%$ confidence interval $(\mathrm{CI})=0.31-1.44)$ and $\mathrm{M} 5(P=0.0123, \mathrm{SMD}=0.88,95 \%$ $\mathrm{CI}=0.19-1.58)$ across the different treatments. Importantly, this association remained when clinical subtypes of IBD (Extended Data Fig. 2d) and intestinal location (Extended Data Fig. 2e) were analyzed separately.

To determine whether the associations with nonresponse are uniform across the genes in modules M4 and M5 or are driven by a small number of them, we compared the contribution of genes by ranking their individual power to predict nonresponse to anti-TNF and corticosteroid therapies. Again, the majority of genes from modules M4 and M5 were among the top predictors of nonresponse to both anti-TNF and corticosteroid therapy relative to genes in other modules (Fig. 1e,f and Supplementary Table 4). Thus, M4 and M5 reflect a coordinated shift in the expression of all their constituent genes in relation to therapy nonresponse across multiple IBD medications.

Coexpression signatures are represented by histopathologic features. In addition to being highly expressed in tissues from therapy nonresponders, modules M4 and M5 also demonstrated the strongest correlation with histologic inflammation (Nancy index) in the discovery cohort ${ }^{13}$ (Fig. 1a). Using an additional clinical cohort of Oxford UC patients (Extended Data Fig. 3), we confirmed that the Nancy index (Fig. 2a), but not other clinical or endoscopic measures (Extended Data Fig. 4a), is higher in nonresponders to anti-TNF therapy before the start of treatment.

Given the foregoing, we postulated that the observed gene coexpression patterns might also reflect histopathologic features associated with IBD (Extended Data Fig. 4b). First, we examined the correlation of quantified histopathologic features with each other (Extended Data Fig. 4c). The only strong positive correlations observed were between cryptitis/crypt abscess and architectural distortion/goblet cell depletion, as well as several associations with granulomas, although there were few cases where granulomas were detected. We then looked for correlations between module expression and histologic feature scores from tissue where both were available $(n=36)$. Although several nominally significant associations were observed between modules and various histopathological features (Fig. 2b), only positive correlations between M4/ulceration and M6/lymphoid aggregates remained significant after adjusting for multiple testing ( $P$ adjusted $<0.05$; Fig. $2 b$ ). Notably, the relation of these two inflammation-associated modules was almost orthogonal, each correlating with only one of the features (Fig. 2c). Despite not reaching significance after correction, M5 also correlated strongly with both ulceration and cryptitis/crypt abscesses (Fig. 2c). We confirmed the associations of M4 and M5 with ulceration in an independent pediatric cohort $(n=172)$ containing inflamed tissues from patients with UC or $\mathrm{CD}^{17,18}$ (Extended Data Fig. 4d). In that dataset, $11 \%$ of all patients with IBD showed high M4/M5 tissue expression (Extended Data Fig. 4e). Similar to our dataset, M6 expression was not significantly different by ulceration status in the pediatric cohort, although we noted that overall M6 expression was also much lower in those biopsy samples (Discussion). In line with the association of M4/M5 with therapy nonresponse, we were able to demonstrate that the extent of histologic ulceration is significantly higher in nonresponders to anti-TNF therapy before the start of treatment (Extended Data Fig. 4f; subcohort of the UC patient cohort used in Extended Data Fig. 3).

The almost orthogonal relation of M4/M5/ulceration with M6/ lymphoid aggregates suggested that these may represent distinct 
a

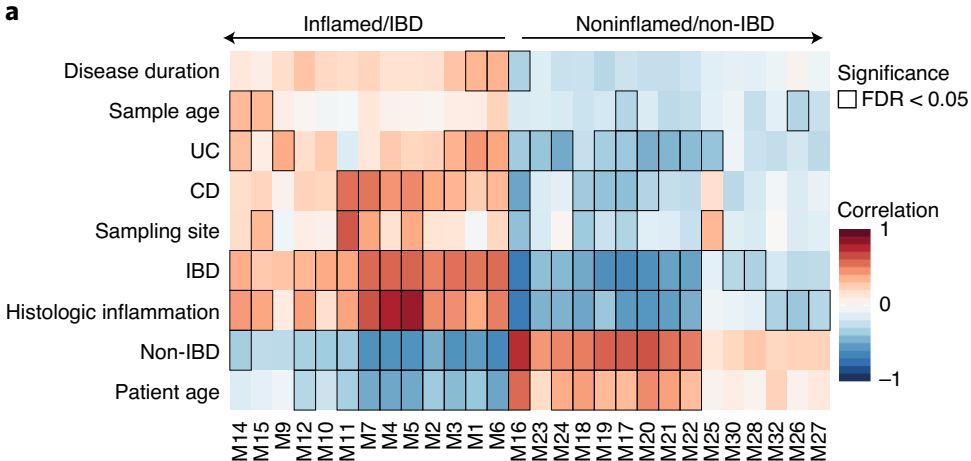

b

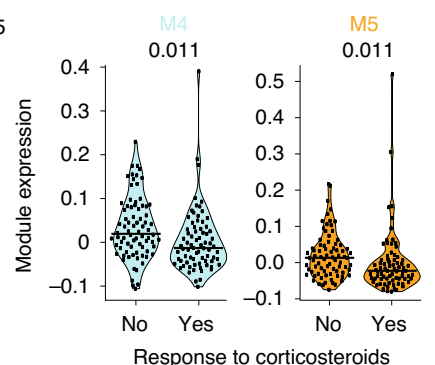

c

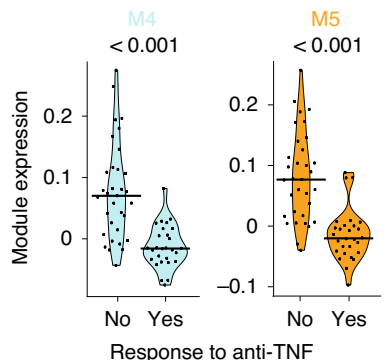

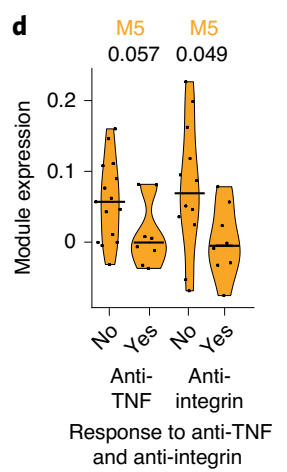

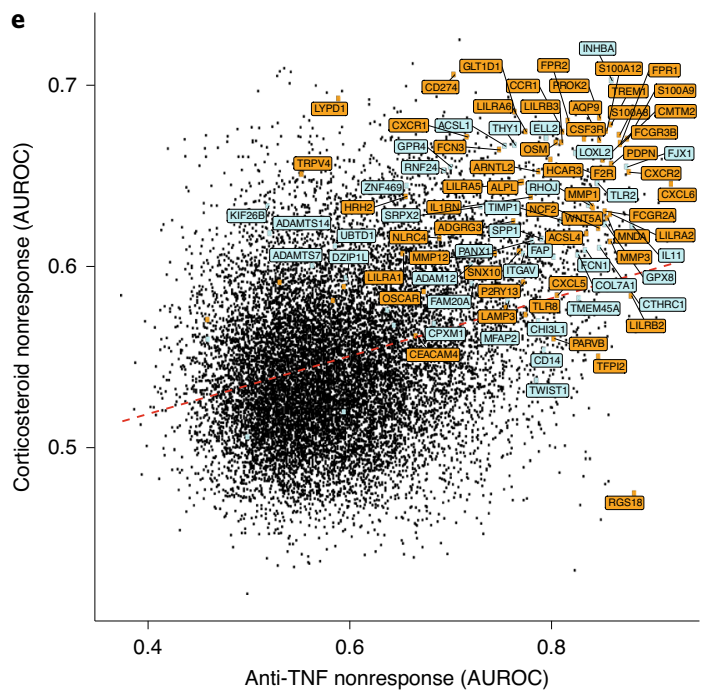

f

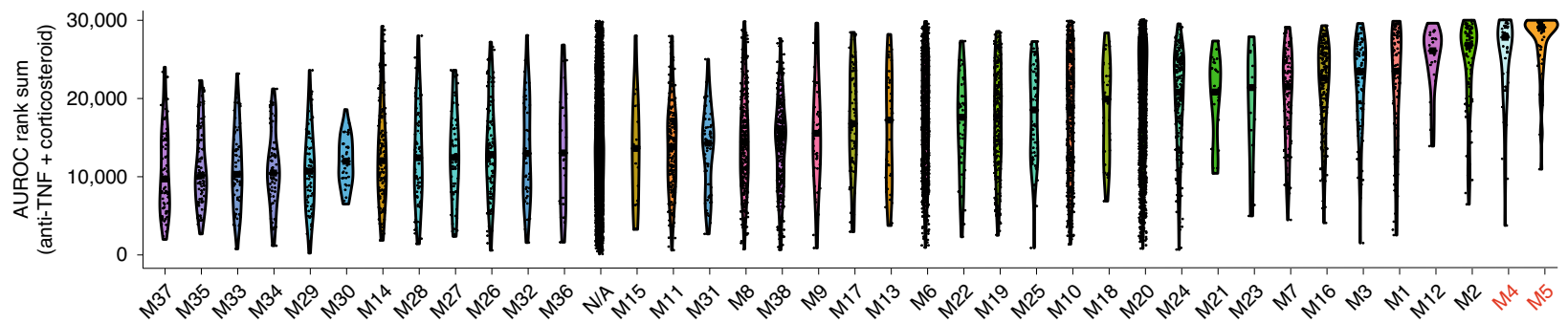

Fig. 1 | Identification of gene coexpression signatures of inflammation associated with patient nonresponse to multiple different IBD therapies. a, Pearson correlation between module eigengenes and clinical and histologic metadata in IBD $(n=41)$ and non-IBD $(n=39$, normal adjacent to CRC) tissues within the discovery cohort; all modules/features with at least one significant association are shown; bordered squares indicate significant correlations (false-discovery rate (FDR) $P<0.05$, asymptotic two-tailed $P$ values estimated from Pearson coefficients). Exact $P$ values are given in Supplementary Table 1. b-d, Expression of modules M4 and M5 (eigengene value) in nonresponders and responders before the start of administration of either corticosteroid ( $\mathbf{b}^{10}, n=206$ patients), anti-TNF (c, $\mathbf{d}^{15,16}, n=61$ patients) or monthly anti-integrin therapy ( $\mathbf{d}^{16}, n=20$ patients) (two-tailed Mann-Whitney $U$-test, FDR adjusted $P$ values; these were post hoc to ANOVA comparisons across the various treatment regimens in ref. ${ }^{16}$ (d). Exact FDR adjusted $P$ values $(\mathbf{c}): \mathrm{M}_{4}, 2.7 \times 10^{-6} ; \mathrm{M}^{-}, 4.7 \times 10^{-7}$. e, Performance (area under the receiver operator curve (AUROC)) of individual genes for prediction of nonresponse to corticosteroid ( $y$ axis) and anti-TNF ( $x$ axis) therapy; genes in M4 and M5 are labeled and highlighted turquoise and orange, respectively. $\mathbf{f}$, Violin plots showing gene rank based on their predictive power (AUROC) for response to both anti-TNF and corticosteroid therapy, comparing all modules as detected in the WGCNA analysis. Combined ranks represent the sum of each gene's ranks in the separate corticosteroid and anti-TNF analyses (their ranks on the $x$ and $y$ axes in $\mathbf{e}$ ).

underlying inflammatory processes dominant in a given patient's tissue. To investigate this, we grouped patients by unsupervised clustering on module M4/M5/M6 expression to determine the relative proportion of patients belonging to these groups. This yielded four groups: M4/M5 high expression (21.7\% of patients), M6 high expression, M5-only high expression and M4/M5/M6 low expression (each $26.1 \%$ of patients) (Fig. 2d). The M4/M5-high group displayed significantly increased expression of $I L 1 B$ compared to the remainder of the patients (Fig. 2e, red). However, neither ITGA4/ITGB7 (encoding integrin subunits targeted by anti-integrin therapy), N3RC1 (targeted by corticosteroids) nor TNF (targeted by anti-TNF) expression was increased in the tissue of these patients (Fig. 2e). By contrast, high expression of module M6 was linked to increased expression of ITGA4 and N3RC1, as well as CCL19, CCL21 and CXCL13 but not TNF (Fig. 2e, blue). Patients high in M5 expression only did not demonstrate significant changes in 

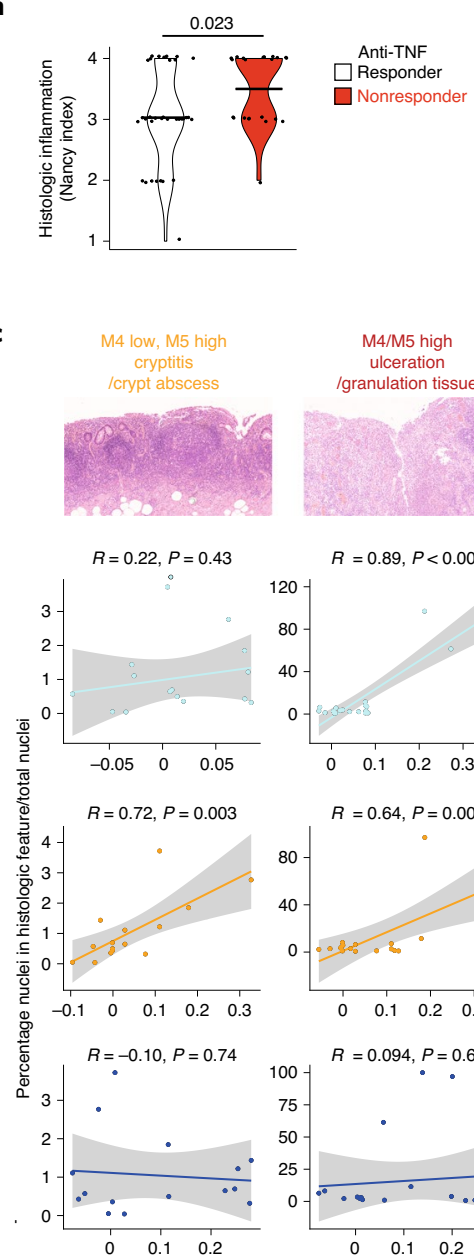

b

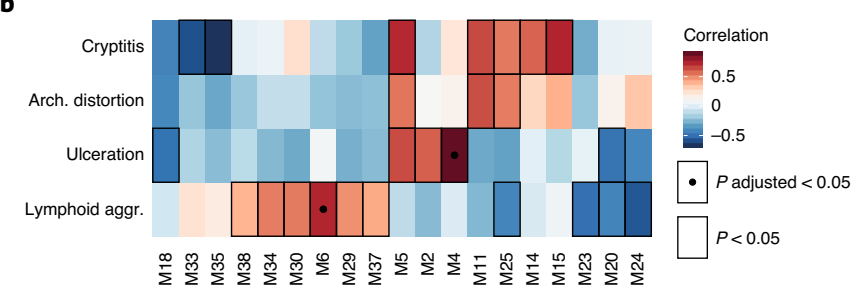

d
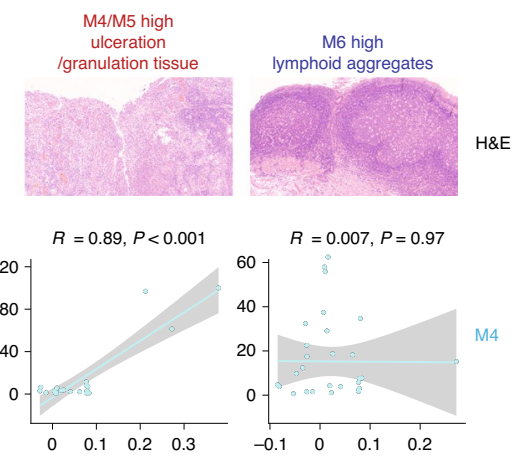

$\mathrm{H} \& \mathrm{E}$
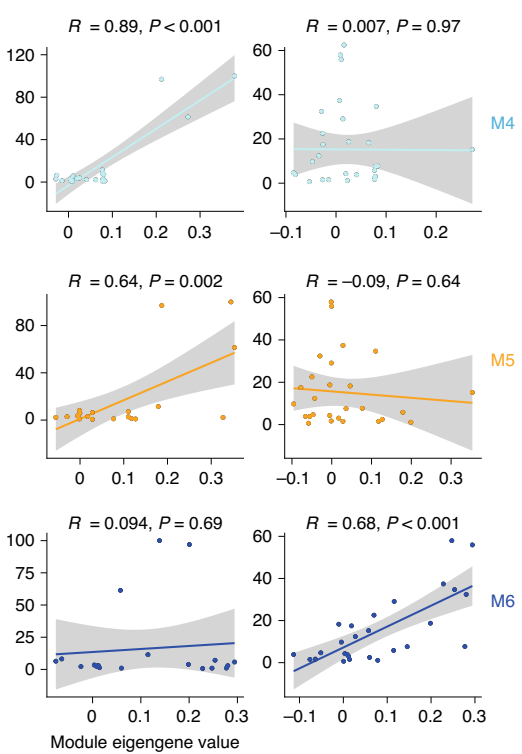
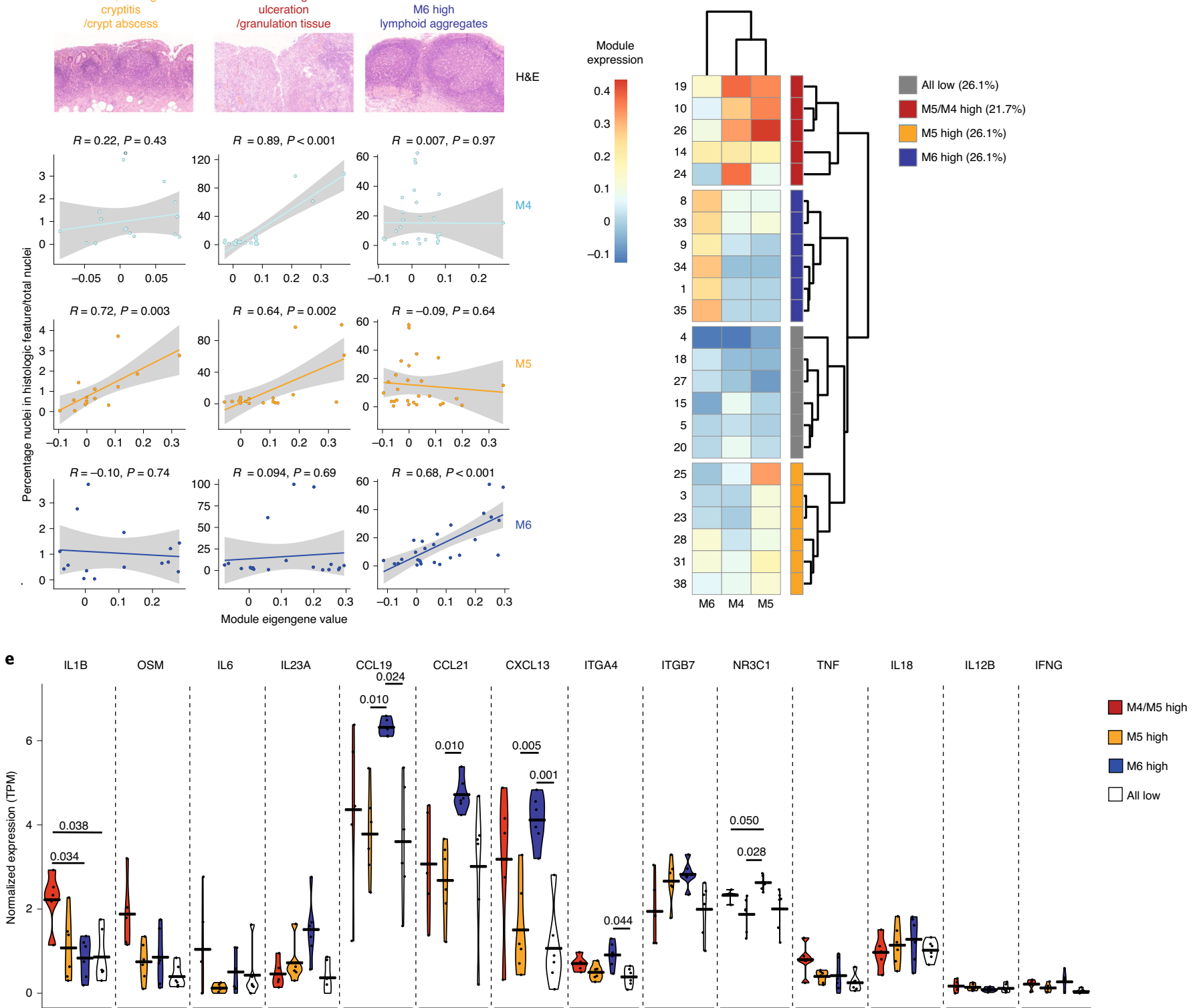

Fig. 2 | Coexpression modules linked with therapy nonresponse represent distinct histopathologic features. a, Nancy histologic scores in responders ( $n=35 \cup C$ ) and nonresponders ( $n=21 \cup C$ ) to anti-TNF therapy within 3 months before the start of treatment (horizontal bars indicate geometric mean, two-tailed Mann-Whitney $U$-test $P$ value is given). b. Heatmap of correlations between module eigengene expression and histological features quantified across tissues from IBD patients in the discovery cohort (where histological features could be quantified). Nominally significant associations $(P<0.05)$ are

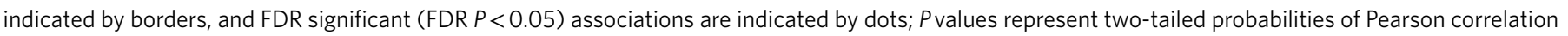
coefficients. Exact $P$ values can be obtained when rerunning the analysis on https://github.com/microbialman/IBDTherapyResponsePaper. c, Scatter plots showing eigengene expression of M4, M5 and M6 versus selected quantified histologic features in tissue samples from patients in the discovery cohort with IBD (linear model fit, error bands show 95\% Cl; P values represent two-tailed probabilities of Pearson correlation coefficients). d, Classification of M4/ M5-high-tissue $(n=5), M 5$ only-high-tissue $(n=6)$, M6-high-tissue $(n=6)$ and M4/M5/M6 ( $n=6)$-low-tissue patients in the discovery cohort, based on hierarchical clustering of module eigengene values from inflamed tissue samples. e, Normalized expression (TPM) of cytokine and therapeutic target genes that were reliably (in $>50 \%$ of samples) detected in the discovery cohort. The expression of these genes is compared in the M4/M5-high tissue (red), M5

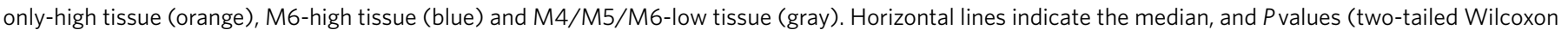
rank-sum test, adjusted for multiple testing) for each comparison are given if significant $(P<0.05)$. Arch., architectural; aggr., aggregates. 
cytokine/therapeutic target signatures (Fig. 2e, orange). Therefore patient responses to specific treatments might be determined by the nature of the inflammatory pathology in the tissue.

High M4/M5 expression reflects neutrophil infiltrates, fibroblast activation and epithelial cell loss. In silico cell type deconvolution showed that M4 and M5 were predominantly associated with stromal cells including fibroblasts and granulocytes, such as neutrophils (Extended Data Fig. 2b). Projection of modules M4 and M5 onto single-cell transcriptomic datasets derived from inflamed and noninflamed $\mathrm{CD}^{6}$ and UC patient tissue ${ }^{7}$ suggested that module M4 reflects the presence of 'activated/inflammatory fibroblasts' whereas module M5 reflects 'myeloid cells/inflammatory monocytes' (Fig. 3a,b).

Gene expression analysis of FACS-sorted cell populations from inflamed tissue from patients with IBD confirmed that several M4/M5 genes were highly expressed in CD16 $6^{\text {hi }}$ neutrophils and podoplanin $(\mathrm{PDPN})^{+} \mathrm{THY}^{+}{ }^{+}$stromal cells (Extended Data Figs. 5a,b and 3 c; see Extended Data Fig. 6 for patient cohort details). Pathway analysis on genes upregulated in either cell type (see Supplementary Table 5 for differential gene expression analysis) demonstrated that neutrophils were enriched in antimicrobial and tissue-toxic granule biology when compared to MNPs that were mostly defined by genes belonging to the antigen presentation pathway (Extended Data Fig. 5d). Stromal cells were enriched in many genes assigned to extracellular matrix pathways (Extended Data Fig. 5d). Of all differentially expressed genes between cell types, 35,28 and $4 \%$ of all genes contained in M4/M5 were significantly enriched in stromal cells, neutrophils and MNPs, respectively (Supplementary Table 5 and Fig. 3c). The enrichment of many M4 and M5 genes in sorted neutrophils explained the high correlation of modules M4 and M5 with the Nancy index (Fig. 1a), which is weighted by the abundance of neutrophils ${ }^{13}$.

To assess whether changes in whole-tissue gene expression reflect changes in cellular numbers, we used flow cytometry to test whether neutrophil and fibroblast counts correlated with M4 and M5 tissue expression (see Extended Data Fig. 5c,e for gating strategy and classification of tissues by M4/M5 expression). The percentage of neutrophils was significantly increased (up to tenfold) in M4/M5-high tissues while the percentage of stromal cells remained unchanged (Fig. 3d). Additionally, epithelial cells were significantly decreased in M4/M5-high tissues (Fig. 3d). Neutrophils accounted for up to $38 \%$ of total live cells in the M4/M5-intermediate and -high groups, whilst the percentage of MNPs was much lower $(<5 \%)$ (Fig. 3d). Furthermore, M4/M5 genes significantly enriched in neutrophils and stromal cells, but not in MNPs, demonstrated the highest predictive power for nonresponse to anti-TNF and corticosteroids (Fig. 3e).
We next quantified the presence of neutrophils, stromal cells and MNPs in situ in resected inflamed tissue from patients with IBD (Fig. 3f). Again, inflamed IBD tissues with high expression of M4 and M5 (see Extended Data Fig. $5 f$ for classification) demonstrated a higher percentage of neutrophil elastase (NE)- and calprotectin (S100A8/A9)-positive cells, but not PDPN-positive stromal cells or CD68 ${ }^{+}$MNPs (Fig. 3g).

High M4/M5 expression in whole tissue thus reflects ulceration characterized by a predominance of neutrophil infiltration, expression of genes characteristic of activated fibroblasts and loss of epithelial cells.

High M4/M5 expression represents neutrophil-attracting fibroblasts and endothelial/perivascular cell expansion. Since we did not observe a change in stromal cell numbers in M4/M5-high tissues (Fig. 3d,g), we hypothesized that the detected stromal signatures could have arisen from altered activation states (including the upregulation of PDPN). We applied scRNA-seq to EPCAM-CD45intestinal stromal cells from endoscopic biopsies of inflamed tissue from patients with UC $(n=7)$ and tissue from healthy donors $(n=4)$ (see Extended Data Fig. 6 for patient cohort details), and compared tissues with low, intermediate and high M4/M5 expression (Fig. 4a,b and Extended Data Fig. 7a). As expected, tissues from all healthy donors were M4/M5 low, as well as the tissue from one patient with IBD who had a low histologic inflammation score (Nancy score $=1$ ). We integrated all single-cell datasets, accounting for interpatient and technical batch effects ${ }^{19}$, and identified six stromal clusters. These were assigned to endothelial cells $\left(A C K R 1^{+} C D 34^{+}\right)$, pericytes $\left(\mathrm{NOTCH}^{+} \mathrm{MCAM}^{+}\right)$, myofibroblasts $\left(\mathrm{MYH} 11^{\mathrm{hi}} A C T G 2^{+}\right)$and three clusters of fibroblasts: PDGFRA ${ }^{\text {hi }} P D P N^{\text {lo }} S O X 6^{+}\left(\mathrm{PDGFRA}^{+}\right)$ fibroblasts, $P D G F R A^{\text {lo }} P D P N^{\text {lo }} A B C A 8^{\text {hi }}\left(A B C A 8^{+}\right)$fibroblasts and $C D 90^{\text {hi }} P D P N^{\text {hi }} P D G F R A^{\text {lo }} A B C A 8^{\text {lo }} F A P^{+ \text {‘ }}$ inflammatory' fibroblasts, based on the top differentially expressed markers and previously described annotations ${ }^{6,7,20}$ (Fig. 4c and Supplementary Table 6). $P D P N$ was expressed by myofibroblasts and all three fibroblast clusters, with the highest expression found in inflammatory fibroblasts. THY1 (CD90) was highly expressed in pericytes and inflammatory fibroblasts, but at lower levels in $\mathrm{ABCA} 8^{+}$fibroblasts (Fig. 4c).

In situ localization of stromal cells in noninflamed large (colon) and small (ileum) intestinal tissue confirmed that PDGFRA ${ }^{+}$and $\mathrm{ABCA} 8^{+}$fibroblasts represent two spatially distinct fibroblast subsets (Fig. 4d and Extended Data Fig. 7b). Costaining of PDPN and THY1 with markers of fibroblasts (PDGFRA, ABCA8), pericytes (MCAM) and endothelial cells (PECAM1) confirmed that multiple stromal subsets express these markers to varying extents (Fig. 4d,e and Extended Data Fig. 7c,d). Notably, THY1 formed a gradient of staining intensity from the perivascular niche toward the lamina propria (as recently described in ref. ${ }^{21}$ ), being expressed by both

Fig. 3 | High expression of modules M4 and M5 reflects neutrophil infiltrates, activated fibroblasts and epithelial cell loss. a,b, Expression of modules M4 and M5 in cell clusters detected by scRNA-seq in tissues of patients with $\mathrm{CD}^{6}$ (a) and $U C^{7}(\mathbf{b})$. c, Heatmap of the levels of expression (TPM values, $z$-score, Manhattan distance clustering) of all genes contained within M4 and M5 in THY1+PDPN ${ }^{+}$stromal cells, CD16 ${ }^{\text {hi }}$ neutrophils and CD14 ${ }^{+} H L A-D R^{ \pm}$ MNPs, FACS-sorted from $n=13, n=12$ and $n=9$ inflamed IBD patient tissues, respectively. Genes are ordered by log fold change of significant enrichment (Padjusted $<0.05$, two-tailed Wald test; Supplementary Table 5) in a cell type. d, FACS cell type percentages in tissue isolates from patients with IBD, classified into low (white, $n=3$ ), intermediate (interm.) (orange, $n=7$ ) and high (red, $n=4$ ) expression of M4/M5 (Extended Data Fig. 3e). Pie-charts ( ${ }^{*}$ denotes significantly different between groups, post hoc two-way ANOVA adjusted $P$ values) show medians across samples, and violin plots individual samples. e, Violin plots showing the combined rank of genes based on their predictive power (AUROC) for response to both anti-TNF and corticosteroid therapy, comparing genes significantly enriched in neutrophils, stromal cells, MNPs or neither (Supplementary Table 5). Horizontal lines indicate the median; adjusted $P$ values are given (two-tailed Wilcoxon test; exact $P$ values are $2.7 \times 10^{-5}$ and $2.1 \times 10^{-4}$ for neutrophil versus nonspecific and neutrophil versus stroma, respectively). f, Illustrative (of samples quantified in $\mathbf{g}$ ) IHC staining (DAB, counterstain hematoxylin) of PDPN, NE, S100A8/A9 and CD68 in serial sections of tissues from patients with IBD classified as low, intermediate or high for M4/M5 whole-tissue gene expression (Extended Data Fig. 3f) Scale bars, $200 \mu \mathrm{m} . \mathbf{g}$, Automated quantification (percentage of positively stained cells of total cells detected in inflamed areas) of IHC stainings shown in f. Each staining was quantified on inflamed tissue sections with low $(n=15)$, intermediate $(n=13)$ and high $(n=12)$ M4/M5 whole-tissue gene expression (Extended Data Fig. 3f). Post hoc two-way, two-tailed ANOVA adjusted $P$ values are given, where significant. 
$\mathrm{ABCA}^{+}$fibroblasts and $\mathrm{MCAM}^{+}$pericytes, as well as by cells in the muscular layer of the submucosa (Fig. 4d,e and Extended Data Fig. $7 b, c)$.

In the single-cell dataset, the percentage of inflammatory fibroblasts, pericytes and endothelial cells was increased in the M4/M5-intermediate and -high patient groups at the expense of $\mathrm{ABCA}^{+}$and PDGFRA ${ }^{+}$fibroblasts (Fig. 4a,b and Extended Data Fig. 7d). FACS analysis verified that $\mathrm{PECAM1}^{+}$endothelial cell and
$\mathrm{PDPN}^{+} \mathrm{FAP}^{+}$inflammatory fibroblast frequencies were increased within the stromal compartment in inflamed compared to noninflamed adjacent tissue (Extended Data Fig. 7e).

To determine which of those clusters contributed most to M4/ M5 expression, we projected the modules onto our scRNA-seq data. Notably, the highest expression of M4 was detected in the inflammatory fibroblast cluster, suggesting the emergence of this cell cluster as an underlying process in M4/M5-high IBD patient tissue (Fig. 5a).

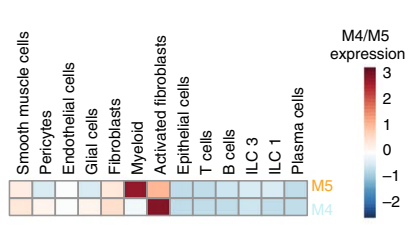

b

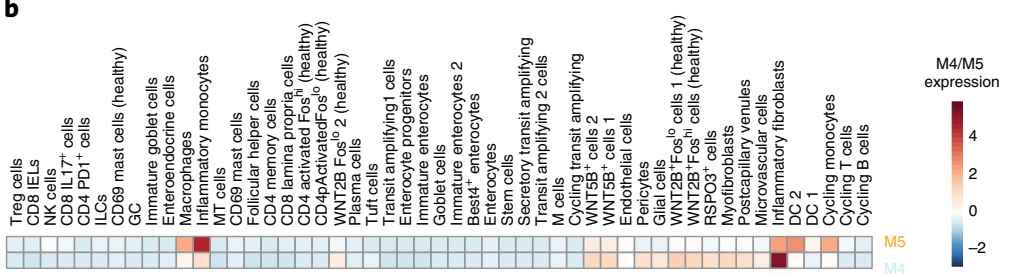

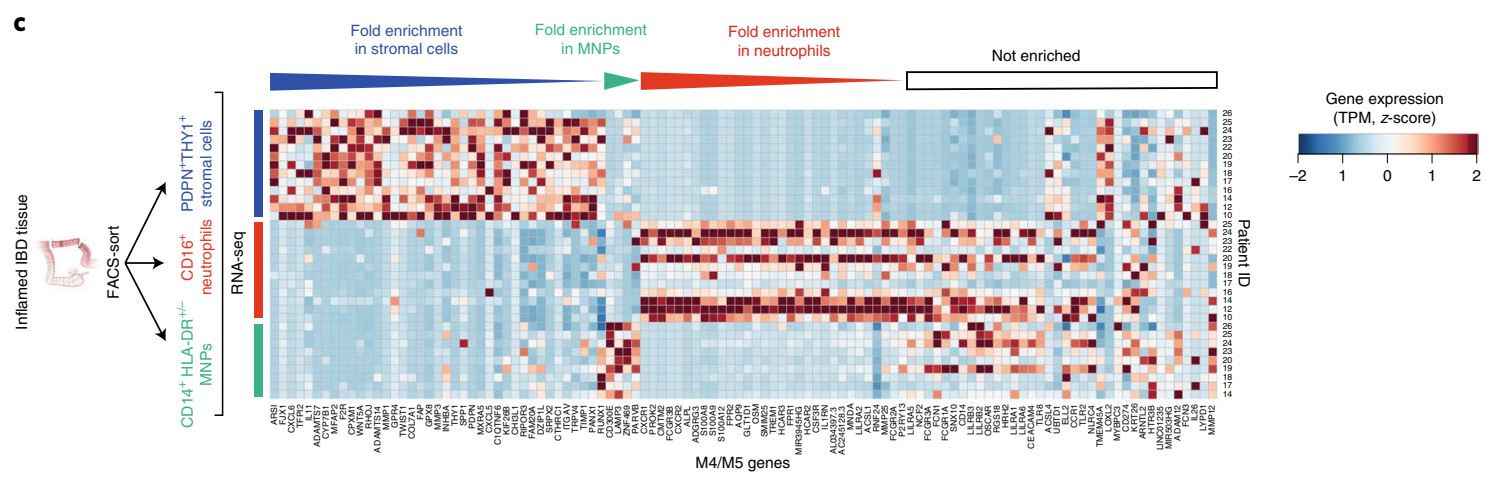

d
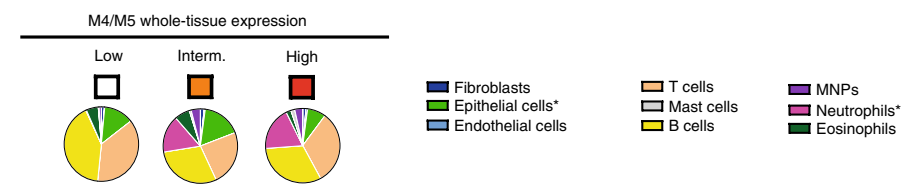

$\mathbf{e}$
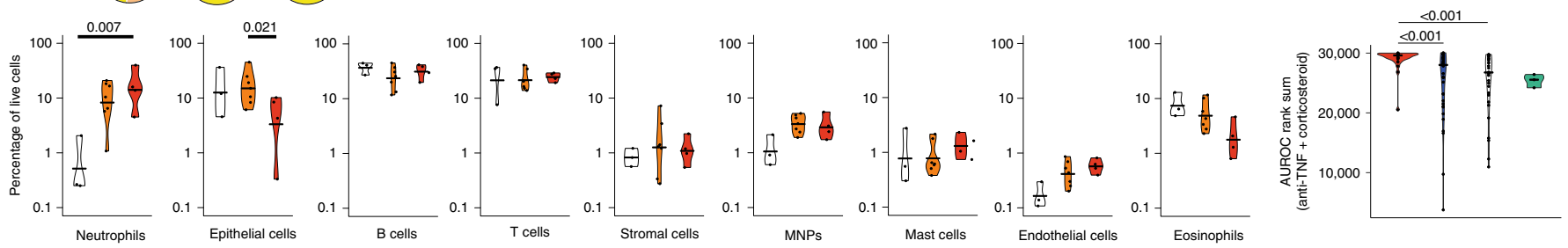

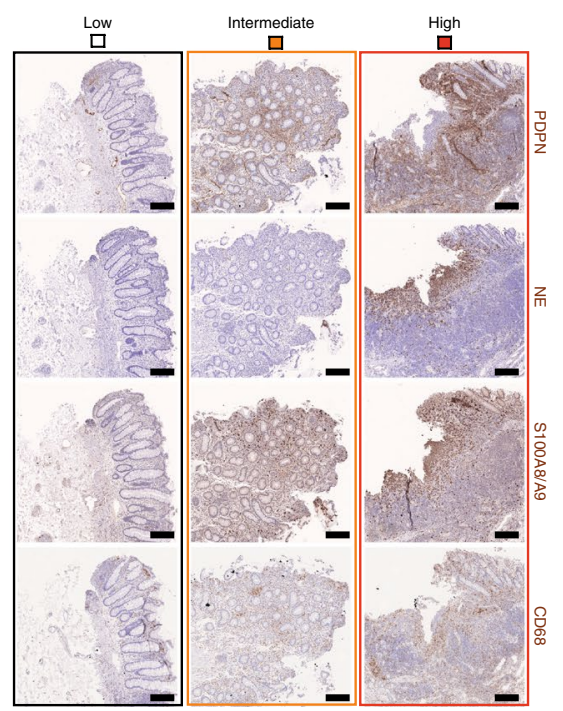

$\mathbf{g}$
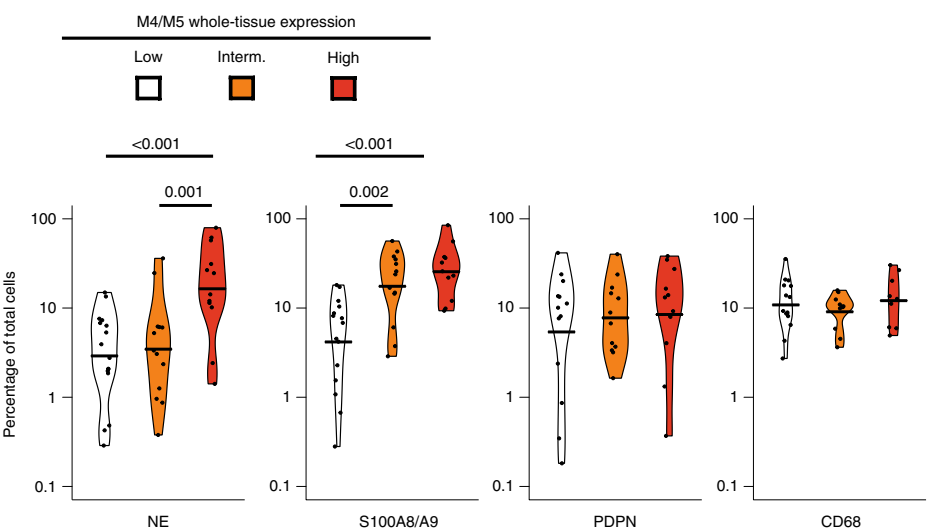
a

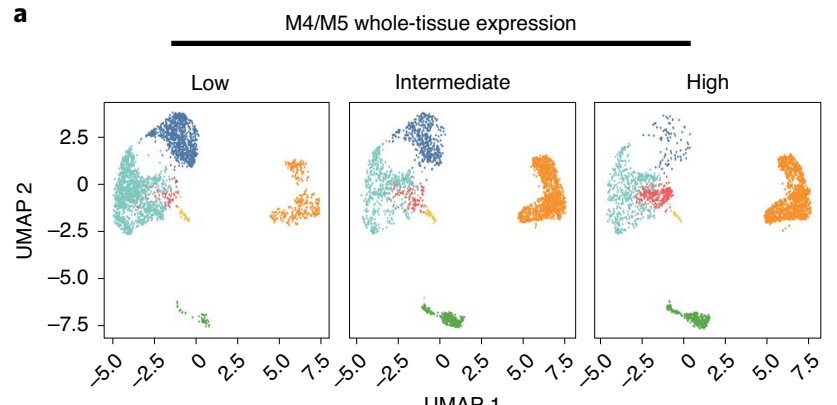

Cell clusters

PDGFRA ${ }^{+}$fibroblasts

ABCA $8^{+}$fibroblasts

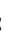

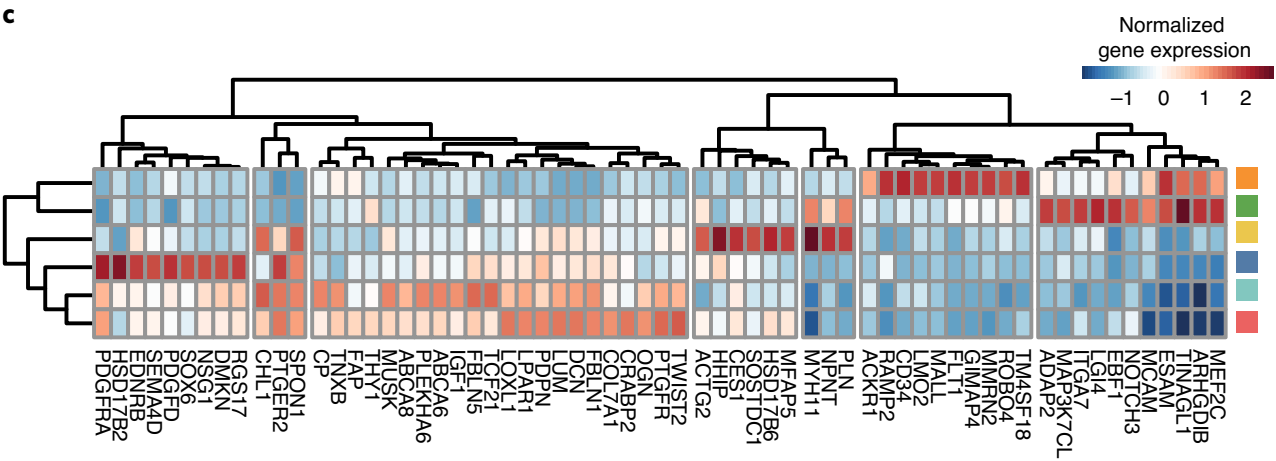

d
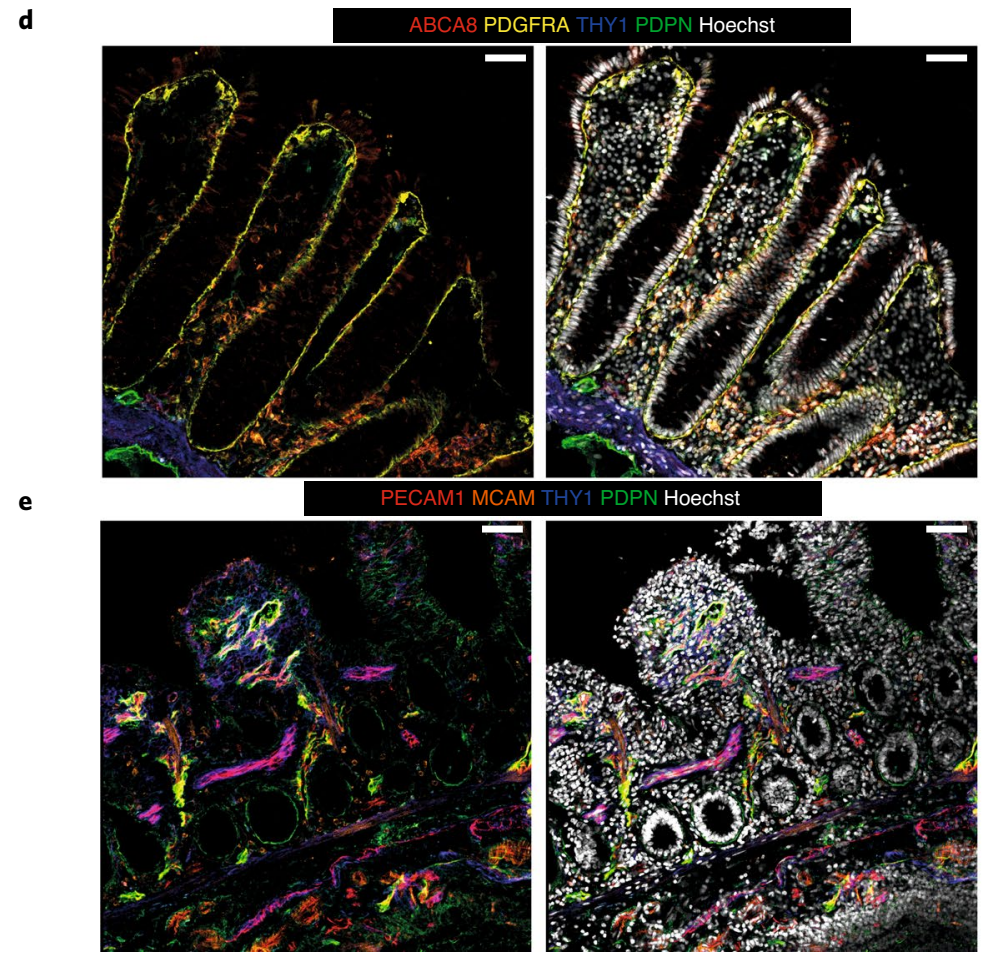

b M4/M5 whole-tissue expression

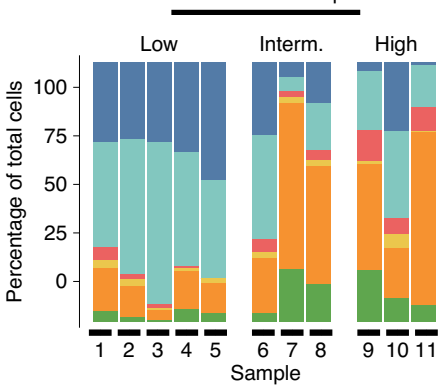

Endothelial cells

Pericytes
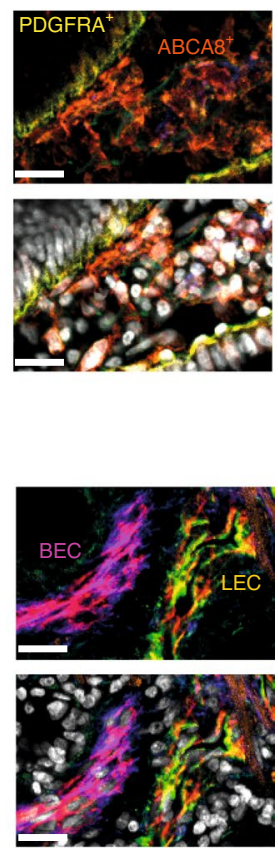

Fig. 4 | Stromal architecture of the large and small intestine in health and disease. a, UMAP of stromal clusters identified by Harmony in stromal compartments, FACS-sorted from healthy donors and tissue from patients with IBD and low $(n=5)$, intermediate $(n=3)$ and high $(n=3)$ M4/M5 whole-tissue gene expression (Extended Data Fig. 4a). b, Percentage of total stromal cells among cell type clusters in M4/M5-low, -intermediate and -high tissue. c, Heatmap of selected markers of each cellular cluster shown in $\mathbf{a}$, as identified by Harmony. Expression values are normalized $\log _{2}$ fold changes (Wald statistic $\frac{\beta_{g}}{\sigma_{g}}$ ) from DESeq2 analyses (where $\beta_{g}$ is $\log _{2}$ fold change for gene $g$, and $\sigma_{g}$ is estimated standard error of $\log _{2}$ fold change for gene $g$ ). d, Immunofluorescent staining of THY1 (blue), PDPN (green), ABCA8 (red) and PDGFRA (yellow) to visualize the localization of fibroblast subsets in resected tissue from patients with IBD (noninflamed areas). Images are representative of stainings in $n=8$ tissues from $n=4$ patients. Scale bars, $50 \mu \mathrm{m}$ ( $20 \mu \mathrm{m}$ in zoomed images). e, Immunofluorescent staining of THY1 (blue), PDPN (green), PECAM1 (red) and MCAM (orange) to visualize the localization of vascular (endothelial) and perivascular cells (noninflamed areas). Images are representative of staining in $n=8$ tissues from $n=4$ patients. Scale bars, $50 \mu \mathrm{m}$ ( $20 \mu \mathrm{m}$ in zoomed images). BEC, blood endothelial cells; LEC, lymphatic endothelial cells. 
Within M4/M5-high tissues, genes encoding neutrophil-targeting CXCR1/CXCR2 ligands CXCL1, CXCL2, CXCL3, CXCL5, CXCL6 and CXCL8 were significantly more highly expressed in inflammatory fibroblasts in comparison to other clusters (Fig. 5b and Supplementary Table 7). Within the cluster of inflammatory fibroblasts, PDPN, FAP, CXCL1, CXCL2, CXCL3, CXCL5, CXCL6 and CXCL8 were also significantly increased in M4/M5-high compared to M4/M5-low and -intermediate tissues, whereas $A B C A 8$ expression was reduced (Supplementary Table 8). Nevertheless, ABCA8 fibroblasts and PDGFRA fibroblasts both still expressed the above-mentioned chemokines in the M4/M5-intermediate and -high groups (Fig. 5b), raising the possibility that the inflammatory fibroblast cluster represents an activation state of ABCA8 fibroblasts and/or PDGFRA fibroblasts. Indeed, trajectory (pseudotime) analysis indicated that inflammatory fibroblasts may represent a transcriptomic state between $\mathrm{ABCA}^{+}$and $\mathrm{PDGFRA}^{+}$fibroblasts (Extended Data Fig. 7f,g), thus potentially arising from either population.

In situ staining confirmed that tissues with dense neutrophil infiltrates ( $\mathrm{NE}^{+}$cells) exhibited the highest level of PDPN on fibroblasts, particularly in areas of profound epithelial cell loss (that is, ulceration) (Fig. 5c). This was associated with the expansion of $\mathrm{THY}^{+}$perivascular cells and blood endothelial vessels $\left(\mathrm{PECAM1}^{+} \mathrm{THY}^{+}{ }^{+} \mathrm{PDPN}^{-}\right.$) (Fig. 5c). Furthermore, colocalization staining revealed that $\mathrm{PDPN}^{+}$fibroblasts expressing FAP (magenta) are found in areas of $\mathrm{NE}^{+}$neutrophil influx (Fig. 5d).

Neutrophil recruitment is driven through fibroblast IL-1R signaling. To identify upstream cytokine drivers of the neutrophil-attractant program in fibroblasts, we stimulated primary stromal cell lines expanded from surgically resected tissue from patients with IBD with a panel of disease-associated cytokines ${ }^{4}$. Of these, only the NF-kB activators IL- $1 \beta$ and TNF- $\alpha$, but not IL- 6 or OSM, induced CXCL5 expression after $3 \mathrm{~h}$ of stimulation (Extended Data Fig. 8a). Furthermore, RNA-seq showed that IL- $1 \beta$ and TNF- $\alpha$ induced strong expression of genes encoding neutrophil-tropic CXCR1 and CXCR2 ligands in fibroblasts, namely CXCL1, CXCL2, CXCL3, CXCL5, CXCL6 and CXCL8 (Extended Data Fig. 8b). In addition, both cytokines induced the inflammatory fibroblast markers PDPN and FAP (Extended Data Fig. 8b). Although TNF- $\alpha$ and IL- $1 \beta$ both induced a chemokine response, the latter was 100 -fold more potent (Extended Data Fig. 8b,c).

We used an ex vivo assay of conditioned medium (CM) generated from patients with IBD to confirm that IL-1 signaling could induce the inflammatory fibroblast phenotype (Fig. 6a). We blocked IL-1 signaling with the IL-1 receptor (IL-1R) antagonist anakinra (Kineret) or TNF signaling with the anti-TNF agent adalimumab (Humira) in CM. Strikingly, only IL-1R, but not TNF, blockade was able to reduce fibroblast activation in this assay (Fig. 6a). This demonstrates that soluble mediators derived from gut-resident cell populations of inflamed IBD tissue activate the neutrophil-attracting fibroblast program in an IL-1R- but not TNF-dependent manner.

Single-cell sequencing showed that inflammatory fibroblasts in M4/M5-high tissue from patients with IBD represented the cell population demonstrating the strongest IL-1 response signature (Fig. 6b; see Supplementary Table 9 for IL-1 gene expression response), suggesting that activation of this pathway may be associated with the poor therapy response observed in those patients. In support, inflammatory fibroblasts and ABCA8 fibroblasts demonstrated the highest fold increase of IL-1 receptor gene expression (IL1R1) in patients with M4/M5-high IBD (Extended Data Fig. 8d). By contrast, the expression of genes encoding TNF receptors (TNFR1 and TNFR2) did not demonstrate this trend (Extended Data Fig. 8d). Consistent with the predominant role of IL-1 in these patients, module M5 was enriched in inflammasome genes (Extended Data Fig. 8e). Additionally, immunohistochemical staining revealed that IL- $1 \beta$ is localized to the ulcer bed and granulation tissue (Fig. 6c, top), but not to noninflamed tissue or that where lymphoid aggregates predominate (Fig. 6d, top). Areas of intense IL-1 $\beta$ labeling also demonstrated intense staining of FAP (Fig. $6 c, d$, middle), suggesting that IL-1R signaling in the ulcer bed drives the inflammatory fibroblast program characterized by FAP expression. Areas of IL-1 $\beta$ and FAP labeling also demonstrated infiltrates of $\mathrm{NE}^{+}$neutrophils (Fig. 6c,d, bottom).

Overall, these results identify IL-1R signaling as a key driver of the inflammatory fibroblast/neutrophil recruitment phenotype that is observed in IBD tissues with a high M4/M5 pathotype.

\section{Discussion}

Our results identify distinct pathotypes within the heterogeneous landscape of IBD that inform on the outcome of therapies. Several genes found in our M4/M5 modules have been associated with nonresponse to anti-TNF therapy or corticosteroids ${ }^{5-7,9-12,15,22}$, but previous studies failed to link these to the identifiable histopathological hallmarks, neutrophil contribution and cytokine drivers of this wider signature. These are important insights with implications for patient stratification in therapeutic targeting.

Our results highlight neutrophils as a major component of the M4/M5 signature associated with nonresponse to several different therapies in IBD. Previous analyses of tissue-level gene expression had linked neutrophils with therapy nonresponse in IBD $^{9,10}$. We have extended those findings by mapping these signatures to intestinal neutrophils isolated from distinct tissue niches within lesions of inflamed intestine. It is also notable that a dominant neutrophil contribution to the biology of inflammation and anti-TNF therapy resistance in IBD is absent from previous scRNA-seq studies where these cells were not analyzed ${ }^{6,7}$. It is not known whether neutrophil accumulation is a cause or a consequence of the damage at sites of tissue ulceration. However, there is evidence that neutrophils can contribute to chronic inflammation through production of extracellular traps and the liberation of reactive-oxygen species ${ }^{23}$. We found that neutrophils are also the major source of OSM expression, a cytokine functionally associated with nonresponse to anti-TNF therapy in $\mathrm{IBD}^{5}$.

In situ localization of inflammatory fibroblasts by PDPN and FAP revealed their proximity to neutrophils in ulcers. We hypothesize that, rather than being a specialized fibroblast subset, inflammatory fibroblasts may represent an activation state of either ABCA8 fibroblasts residing in the lamina propria of the intestine, or subepithelial PDGFRA fibroblasts.

We also observed an expansion of $\mathrm{ACKR}^{+}$endothelial cells alongside activated fibroblasts in M4/M5-high tissues/deep ulcers. Module M2 also strongly correlated with endothelial cells in our in

Fig. 5 | M4/M5 gene expression is associated with neutrophil-attracting fibroblasts and endothelial and perivascular cell expansion. a, UMAP of stromal single-cell profiles showing the different stromal clusters as in Fig. 4a for comparison (top), and the expression level of M4 (middle) and M5 (bottom)

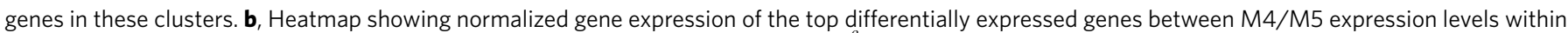
each cell cluster. Expression values are normalized $\log _{2}$ fold changes (Wald statistic $\frac{\beta_{g}}{\sigma_{g}}$ ) from DESeq2 analyses. c, Staining of NE or PECAM1 (red), THY1 (blue) and PDPN (green) in $n=4$ tissues from patients with IBD and with varying grades of neutrophil infiltration. Images are representative of stainings in $n=8$ tissues from $n=4$ patients. Scale bars, $50 \mu \mathrm{m}$. d, Staining of NE (green), FAP (blue) and PDPN (red) in paired inflamed (deep ulcer) and noninflamed tissues. Images are representative of staining in $n=8$ tissues from $n=4$ patients. Scale bars, $200 \mu \mathrm{m}(20 \mu \mathrm{m}$ in zoomed image insert). 
silico predictions, and was the third strongest association with therapy nonresponse. Since endothelial cells did not express high levels of genes coding for neutrophil-chemoattractant CXCLs, we can speculate that inflammatory fibroblasts produce these chemokines, which are then presented by ACKR $1^{+}$endothelial cells to circulating neutrophils to initiate extravasation ${ }^{24}$.

The very specific localization of IL- $1 \beta$ in the areas of epithelial cell damage in ulcers suggests that disruption of the epithelial

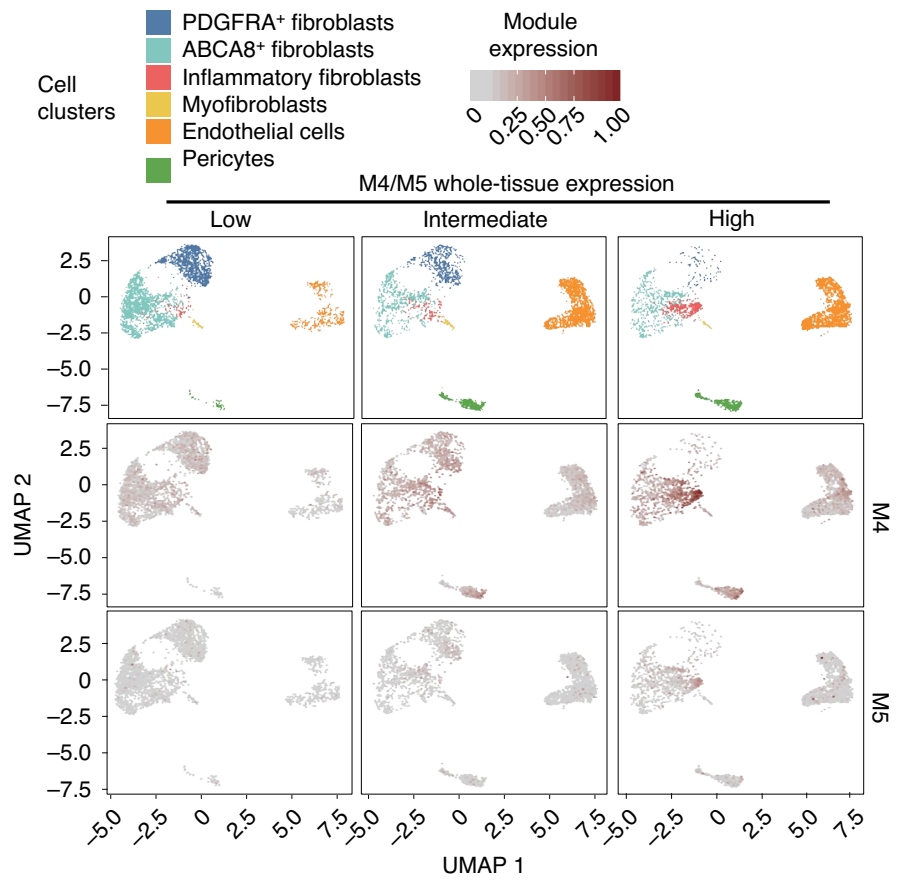

b
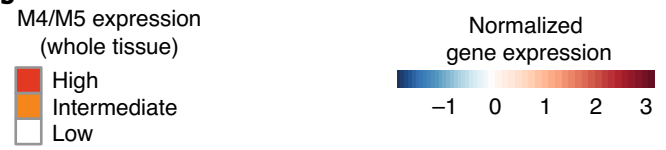

Low

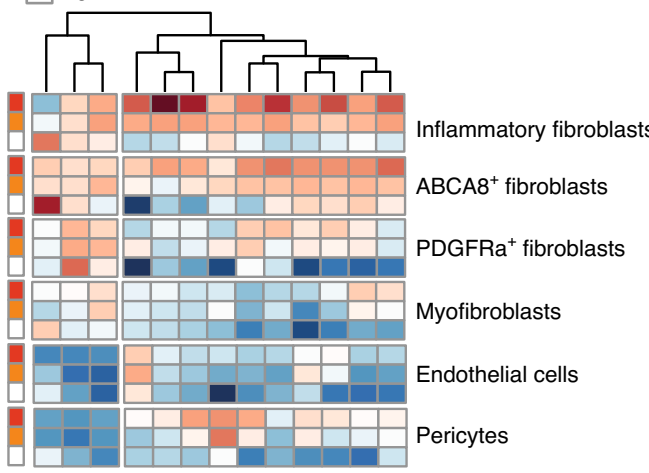

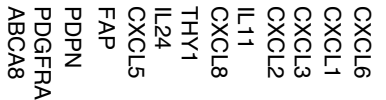

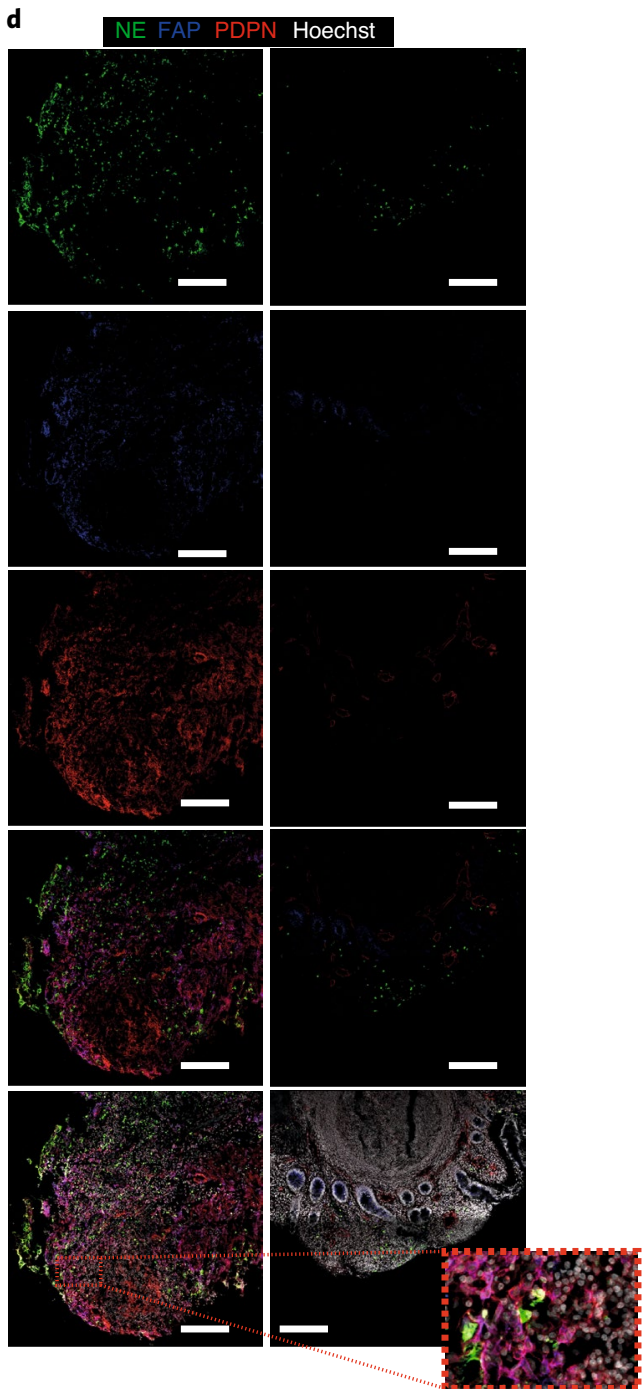

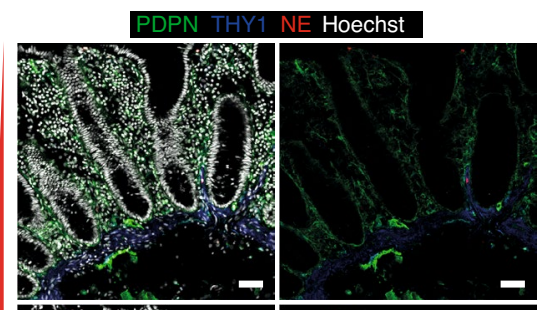
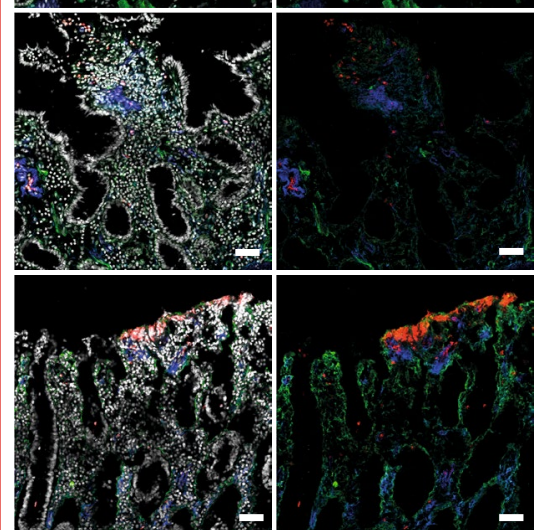

is
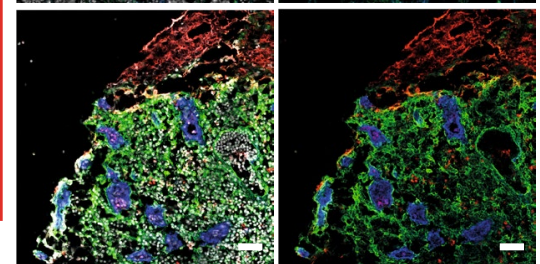
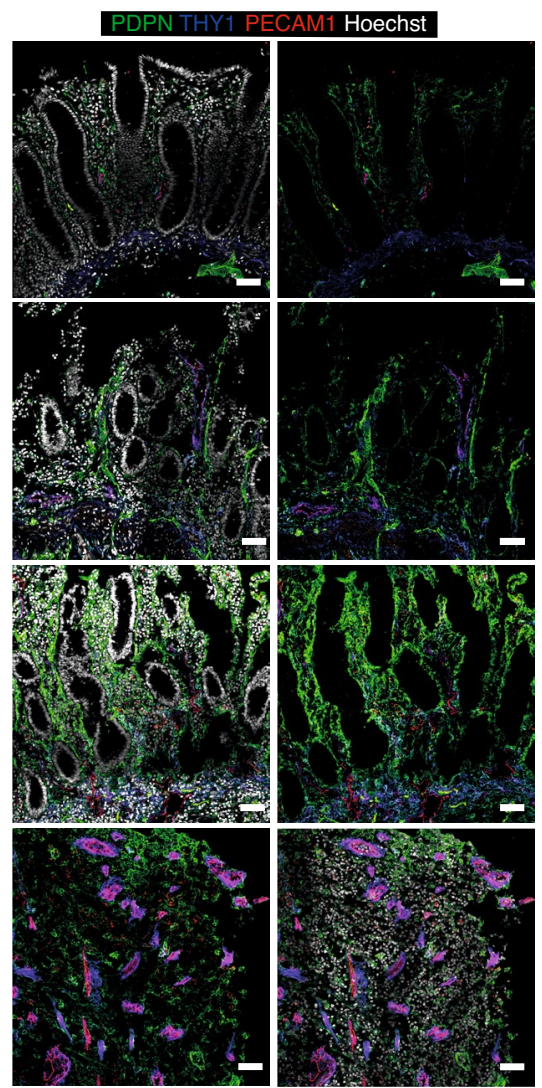

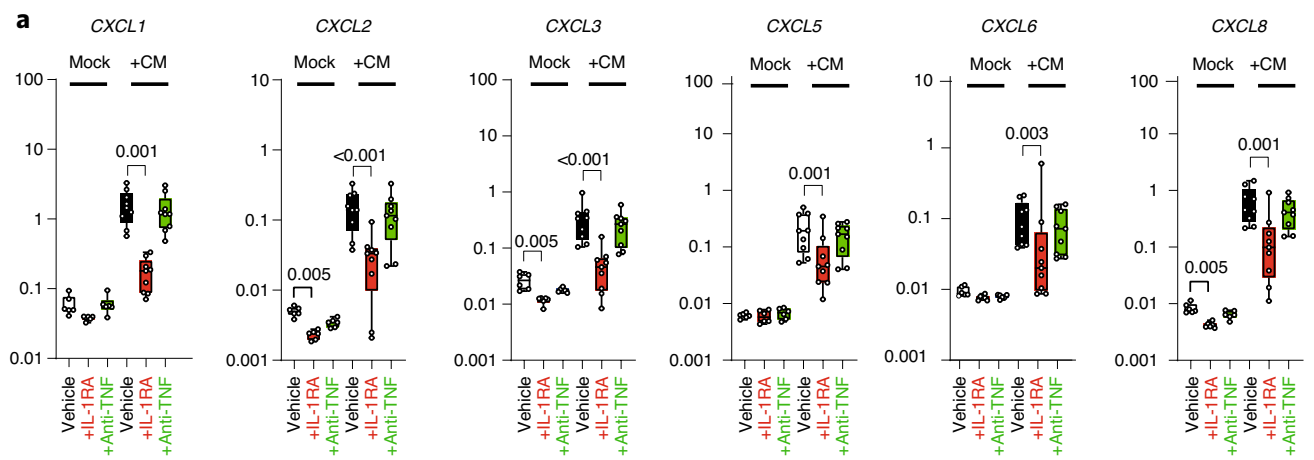

b

M4/M5 expression

C
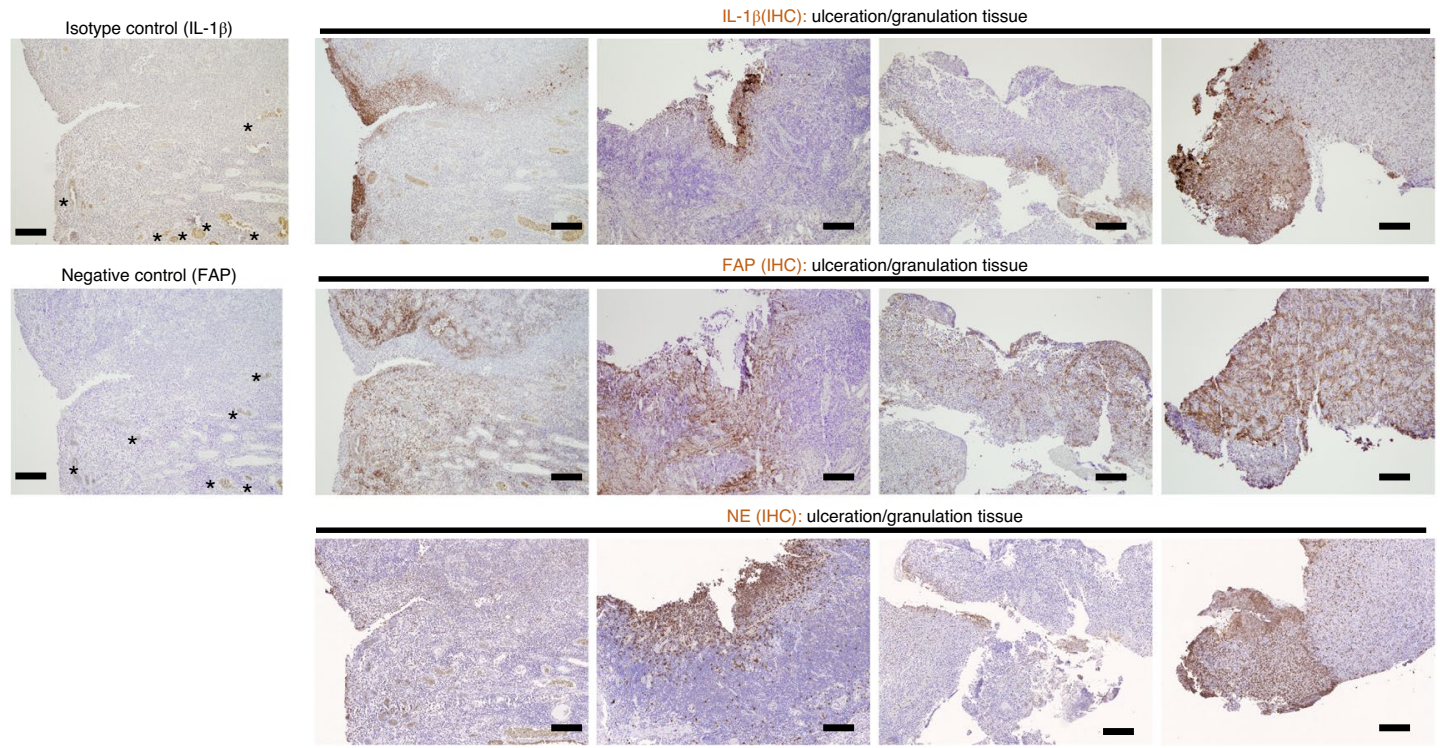

d

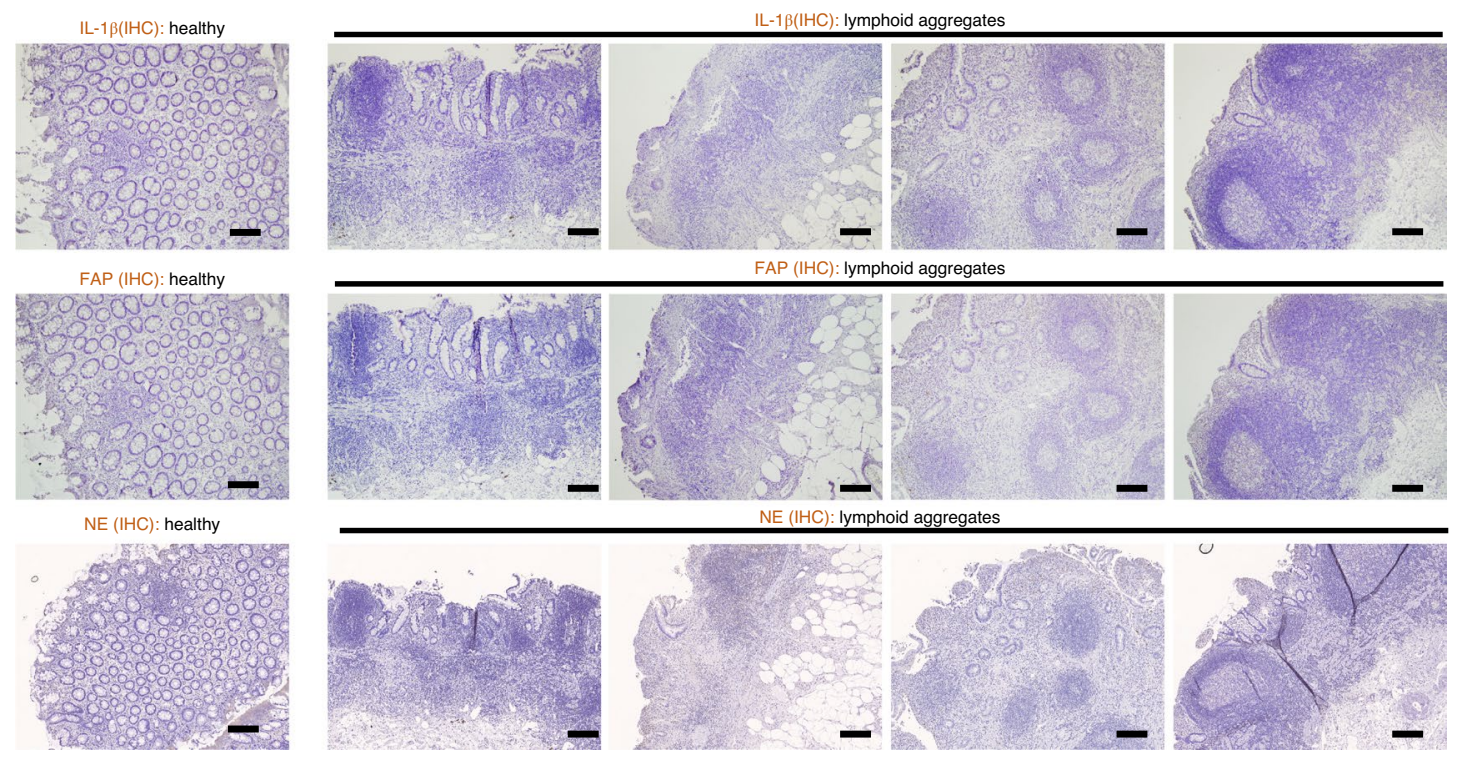

Fig. 6 | Activated inflammatory fibroblasts drive neutrophil recruitment through IL-1R signaling with high levels of IL-1 $\beta$ at sites of ulceration.

a, Ccd18-Co fibroblasts were stimulated for $3 \mathrm{~h}$ with either mock control or conditioned media produced from $n=9$ tissue digests from patients with IBD (CM), without pretreatment (vehicle, PBS) or preincubated with IL-1Ra (anakinra) or anti-TNF (adalimumab). Adjusted Pvalues are shown where significant $(P<0.05)$; two-tailed Friedman test for paired samples. $\mathbf{b}$, Projection of the IL-1 cytokine stimulation response of Ccd18-Co fibroblasts onto stromal cell clusters detected by scRNA-seq (Fig. 4a). Scores were computed as mean $z$-score of IL-1 upregulated genes. c, IHC staining of IL-1 $\beta$, FAP or NE ( $D A B$, counterstain hematoxylin) in $n=8$ different inflamed (infl.) tissue sections of patients with IBD and with prominent ulceration and/or granulation tissue, and in $n=1$ healthy tissue. Scale bars, $200 \mu \mathrm{m}$. * Indicates nonspecific staining of erythrocytes or platelets in vessels. $\mathbf{d}$, Staining as in $\mathbf{c}$, but of inflamed tissue sections from patients with IBD and with dominant lymphoid aggregates. Scale bars, $200 \mu \mathrm{m}$. 
barrier may be a primary event. Early responders to this damage may also include resident MNP, which can produce excessive IL-1 $\beta$ and IL-23 particularly in the context of IL-10 pathway deficiency ${ }^{11}$. Alarmin IL- $1 \alpha$ may similarly contribute to the activation of fibroblasts and initiation of colitis ${ }^{25,26}$, and can be released by necrotic epithelial cells in $\mathrm{IBD}^{27}$. Further studies are required to establish whether the IL-1R-driven activation of inflammatory fibroblasts identified here is dominated by IL- $1 \alpha$ or IL- $1 \beta$ signaling or both.

In addition to the M4/M5-high pathotype, we also identified patients with high M6 tissue expression associated with lymphoid aggregates. The high tissue expression of CCL19/CCL21/CXCL13 suggests that this pathotype reflects the presence of fibroblastic reticular-like cells ${ }^{20}$. The expression of M6 was very low in pediatric-onset UC and CD; this may reflect the different nature of the samples analyzed in the two studies, the latter using endoscopic punch biopsies limited to the mucosa rather than full-thickness surgical specimens. This requires consideration when interpreting lymphoid signals from endoscopic biopsies.

Patients with UC or CD whose tissues show a high M4/M5 signature and ulceration express high amounts of $I L 1 B$ but not NR3C1, ITGA4 or TNF, suggesting they may benefit from blocking of IL-1R rather than TNF to target the neutrophil-attractant program in fibroblasts. Indeed, TNF can promote mucosal healing ${ }^{28}$ and therefore may be deleterious in patients with deep ulceration. Genetic defects in the IL-1 pathway have been linked to anti-TNF nonresponse ${ }^{29}$, and the principle of ameliorating acute intestinal inflammation by blockade of IL-1 signaling has been demonstrated in preclinical models ${ }^{30-32}$. In case studies of Mendelian disease-like IBD with IL-10 deficiency, the blockade of IL-1 signaling can successfully treat intestinal inflammation ${ }^{33,34}$. Surprisingly, large-scale studies of IL-1 blockade in polygenic IBD patient cohorts are lacking although trials in acute severe ulcerative colitis are ongoing ${ }^{35}$.

Our surgical resection samples highlight the heterogeneity of inflammatory lesions in a difficult-to-treat patient group. These data are only a snapshot and do not inform on the evolution and dynamics of the distinct pathotypes identified here. However, the presence of M4/M5-signature-high patients before treatment in a number of prospective cohorts suggests that deep ulceration and high M4/M5 signature can occur independently of therapy failure. Our study does not address whether lymphoid aggregates and ulceration are independent processes or connected states. Notably, the presence of M4/M5 and M6 is not mutually exclusive, and a small number of tissues exhibited both ulceration and lymphoid aggregates. Further understanding of the natural history of these distinct pathotypes and their relationship to disease dynamics will require longitudinal analyses.

In summary, integration of data across biological levels identifies new tissular IBD pathotypes that are defined by different molecular, cellular and histopathologic features and are associated with patient responses to current therapeutics. Currently, subcategories of IBD are classified by high-level phenotypes. A deficit in understanding the cellular and molecular pathotypes in IBD has restricted prescription of therapeutics based on the underlying biologic processes they target, increasing the likelihood of failure. Future trials should consider patient inclusion criteria based on the observations presented here. Our data indicate that IL-1 blockade may benefit those individuals with deep ulceration and who do not respond to several different current therapeutics. This may improve treatment outcomes for patients with IBD by hastening the administration of appropriate interventions and providing an alternative therapeutic target in an area of unmet clinical need.

\section{Online content}

Any methods, additional references, Nature Research reporting summaries, source data, extended data, supplementary information, acknowledgements, peer review information; details of author contributions and competing interests; and statements of data and code availability are available at https://doi.org/10.1038/ s41591-021-01520-5.

Received: 6 February 2021; Accepted: 26 August 2021; Published online: 21 October 2021

\section{References}

1. Uhlig, H. H. \& Powrie, F. Translating immunology into therapeutic concepts for inflammatory bowel disease. Annu. Rev. Immunol. 36, 755-781 (2018).

2. Ungaro, R., Mehandru, S., Allen, P. B., Peyrin-Biroulet, L. \& Colombel, J. F. Ulcerative colitis. Lancet 389, 1756-1770 (2017).

3. Torres, J., Mehandru, S., Colombel, J. F. \& Peyrin-Biroulet, L. Crohn's disease Lancet 389, 1741-1755 (2017).

4. Friedrich, M., Pohin, M. \& Powrie, F. Cytokine networks in the pathophysiology of inflammatory bowel disease. Immunity 50, 992-1006 (2019).

5. West, N. R. et al. Oncostatin M drives intestinal inflammation and predicts response to tumor necrosis factor-neutralizing therapy in patients with inflammatory bowel disease. Nat. Med. 23, 579-589 (2017).

6. Martin, J. C. et al. Single-cell analysis of Crohn's disease lesions identifies a pathogenic cellular module associated with resistance to anti-TNF therapy. Cell 178, 1493-1508 (2019).

7. Smillie, C. S. et al. Intra- and inter-cellular rewiring of the human colon during ulcerative colitis. Cell 178, 714-730 (2019).

8. Huang, B. et al. Mucosal profiling of pediatric-onset colitis and IBD reveals common pathogenics and therapeutic pathways. Cell 179, 1160-1176 (2019).

9. Czarnewski, P. et al. Conserved transcriptomic profile between mouse and human colitis allows unsupervised patient stratification. Nat. Commun. 10, 2892 (2019)

10. Haberman, Y. et al. Ulcerative colitis mucosal transcriptomes reveal mitochondriopathy and personalized mechanisms underlying disease severity and treatment response. Nat. Commun. 10, 38 (2019).

11. Aschenbrenner, D. et al. Deconvolution of monocyte responses in inflammatory bowel disease reveals an IL-1 cytokine network that regulates IL-23 in genetic and acquired IL-10 resistance. Gut 70, 1023-1036 (2021).

12. Gaujoux, R. et al. Cell-centred meta-analysis reveals baseline predictors of anti-TNFalpha non-response in biopsy and blood of patients with IBD. Gut 68, 604-614 (2019).

13. Marchal-Bressenot, A. et al. Development and validation of the Nancy histological index for UC. Gut 66, 43-49 (2017).

14. Aran, D., Hu, Z. \& Butte, A. J. xCell: digitally portraying the tissue cellular heterogeneity landscape. Genome Biol. 18, 220 (2017).

15. Arijs, I. et al. Mucosal gene expression of antimicrobial peptides in inflammatory bowel disease before and after first infliximab treatment. PLoS ONE 4, e7984 (2009).

16. Arijs, I. et al. Effect of vedolizumab (anti-alpha4beta7-integrin) therapy on histological healing and mucosal gene expression in patients with UC. Gut 67, 43-52 (2018).

17. Haberman, Y. et al. Pediatric Crohn disease patients exhibit specific ileal transcriptome and microbiome signature. J. Clin. Invest. 124, 3617-3633 (2014).

18. Loberman-Nachum, N. et al. Defining the celiac disease transcriptome using clinical pathology specimens reveals biologic pathways and supports diagnosis. Sci. Rep. 9, 16163 (2019).

19. Korsunsky, I. et al. Fast, sensitive and accurate integration of single-cell data with Harmony. Nat. Methods 16, 1289-1296 (2019).

20. Kinchen, J. et al. Structural remodeling of the human colonic mesenchyme in inflammatory bowel disease. Cell 175, 372-386 (2018).

21. Wei, K. et al. Notch signalling drives synovial fibroblast identity and arthritis pathology. Nature 582, 259-264 (2020).

22. Arijs, I. et al. Mucosal gene signatures to predict response to infliximab in patients with ulcerative colitis. Gut 58, 1612-1619 (2009).

23. Cassatella, M. A., Ostberg, N. K., Tamassia, N. \& Soehnlein, O. Biological roles of neutrophil-derived granule proteins and cytokines. Trends Immunol. 40, 648-664 (2019).

24. Pruenster, M. et al. The Duffy antigen receptor for chemokines transports chemokines and supports their promigratory activity. Nat. Immunol. 10, 101-108 (2009).

25. Bersudsky, M. et al. Non-redundant properties of IL-1alpha and IL-1beta during acute colon inflammation in mice. Gut 63, 598-609 (2014).

26. Scarpa, M. et al. The epithelial danger signal IL-1alpha is a potent activator of fibroblasts and reactivator of intestinal inflammation. Am. J. Pathol. 185 , 1624-1637 (2015).

27. Boyapati, R. K., Rossi, A. G., Satsangi, J. \& Ho, G. T. Gut mucosal DAMPs in IBD: from mechanisms to therapeutic implications. Mucosal Immunol. 9, 567-582 (2016) 
28. Atreya, R. \& Neurath, M. F. Current and future targets for mucosal healing in inflammatory bowel disease. Visc. Med. 33, 82-88 (2017).

29. Bank, S. et al. Associations between functional polymorphisms in the NFkappaB signaling pathway and response to anti-TNF treatment in Danish patients with inflammatory bowel disease. Pharmacogenomics J. 14, 526-534 (2014).

30. Siegmund, B., Lehr, H. A., Fantuzzi, G. \& Dinarello, C. A. IL-1 beta -converting enzyme (caspase-1) in intestinal inflammation. Proc. Natl Acad. Sci. USA 98, 13249-13254 (2001).

31. Coccia, M. et al. IL-1beta mediates chronic intestinal inflammation by promoting the accumulation of IL-17A secreting innate lymphoid cells and CD4(+) Th17 cells. J. Exp. Med. 209, 1595-1609 (2012).

32. Castro-Dopico, T. et al. Anti-commensal IgG drives intestinal inflammation and type 17 immunity in ulcerative colitis. Immunity 50, 1099-1114 (2019).

33. Levy, M. et al. Severe early-onset colitis revealing mevalonate kinase deficiency. Pediatrics 132, e779-e783 (2013).

34. Shouval, D. S. et al. Interleukin 1beta mediates intestinal inflammation in mice and patients with interleukin 10 receptor deficiency. Gastroenterology 151, 1100-1104 (2016).

35. Thomas, M. G. et al. Trial summary and protocol for a phase II randomised placebo-controlled double-blinded trial of Interleukin 1 blockade in acute severe colitis: the IASO trial. BMJ Open 9, e023765 (2019).

Publisher's note Springer Nature remains neutral with regard to jurisdictional claims in published maps and institutional affiliations.

Open Access This article is licensed under a Creative Commons
Attribution 4.0 International License, which permits use, sharing, adap-
tation, distribution and reproduction in any medium or format, as long
as you give appropriate credit to the original author(s) and the source, provide a link to
the Creative Commons license, and indicate if changes were made. The images or other
third party material in this article are included in the article's Creative Commons license,
unless indicated otherwise in a credit line to the material. If material is not included in
the article's Creative Commons license and your intended use is not permitted by statu-
tory regulation or exceeds the permitted use, you will need to obtain permission directly
from the copyright holder. To view a copy of this license, visit http://creativecommons.
org/licenses/by/4.0/.
(c) The Author(s) 2021

\section{Oxford IBD Cohort Investigators}

Fiona M. Powrie', Simon P. Travis ${ }^{8}$ and Holm H. Uhlig 8,11

A full list of members and their affiliations appears in the Supplementary Information.

\section{Roche Fibroblast Network Consortium}

\section{Francesca Barone ${ }^{14}$, Michael Brenner ${ }^{6}$, Christopher D. Buckley ${ }^{1,14,15}$, Mark Coles $^{1}$, Andreas P. Frei ${ }^{16}$, Kara G. Lassen ${ }^{16}$, Fiona M. Powrie ${ }^{1,15}$ and Soumya Raychaudhuri ${ }^{2,3,4,5,6,13}$}

\footnotetext{
${ }^{14}$ Rheumatology Research Group, Institute for Inflammation and Ageing, College of Medical and Dental Sciences, University of Birmingham, Queen Elizabeth Hospital, Birmingham, UK. ${ }^{15}$ NIHR Birmingham Biomedical Research Centre, University Hospitals Birmingham NHS Foundation Trust, Birmingham, UK. ${ }^{16}$ Roche Pharma Research and Early Development, Immunology, Infectious Diseases and Ophthalmology (I2O) Discovery and Translational Area, Roche Innovation Center Basel, Basel, Switzerland.
} 


\section{Methods}

Statistics and reproducibility. Due to the nature of the study, no statistical method was used to predetermine sample size and experiments were not randomized; $n=15$ samples of bulk sequencing from surgical resections were excluded based on failed quality control (low number of reads, outlier on PCA) (see GSE166928 for flags and https://github.com/microbialman/IBDTherapyResponsePaper). Given the sample nature, technical replication of experiments involving human patient sample material was not carried out because collection of substantially more tissue could not be ethically supported. Patient data were analyzed at the cohort level, using all patients/samples/datapoints, to derive statistics. For experiments with cell lines, replicates are presented and experiments shown only if the experiment was successfully repeated on at least one other occasion. Randomization was not relevant to the clinical findings because this was an observational study. Cell culture wells were randomly allocated to experimental groups. All human samples and cell line treatment groups were blinded to the researchers before data collection, by giving them a unique ID number. Pathologists were blinded for scoring of histopathologic slides. The type of statistical test and experimental group sizes are given in the figure legends.

Patient cohorts and ethics. Patients eligible for inclusion in the discovery cohort were identified by screening surgical programs at Oxford University Hospitals. Samples were obtained from patients undergoing surgical resection of affected tissue for UC, CD or CRC (used as non-IBD controls). All tissue samples included in the study were classified by pathological examination as either macroscopically active inflamed or noninflamed. Additional samples were also obtained from patients with CD and UC, or from healthy individuals by biopsy. All patients and healthy participants gave informed consent and collection was approved by NHS National Research Ethics Service under the research ethics committee reference nos. IBD 09/H1204/30 and 11/YH/0020 (for IBD) and GI 16/YH/0247 (for CRC samples and gut biopsies from healthy individuals). Samples were immediately placed on ice (RPMI 1640 medium) and processed within $3 \mathrm{~h}$. All data were fully anonymized before analyses. For replication of prospective findings in the discovery cohort, public datasets derived from endoscopic tissue samples of patients with IBD were used ${ }^{10,15-17,36}$ (GSE16879, GSE73661, GSE109142, GSE57945, GSE100833).

Isolation of cells from tissue samples. After removal of external muscle and adipose layers, and removal of bulk epithelial cells by repeated washes in PBS containing antibiotics (penicillin/streptomycin, amphotericin B, gentamicin, ciprofloxacin) and $5 \mathrm{mM}$ EDTA (Sigma-Aldrich), tissue from surgical resections was minced using surgical scissors. In the case of endoscopic biopsies, the epithelial wash was omitted. Minced tissue was subjected to multiple rounds of digestion in RPMI 1640 medium containing 5\% fetal bovine serum (FBS), 5 mM HEPES, antibiotics as above and $1 \mathrm{mg} \mathrm{ml}^{-1}$ Collagenase A and DNase I (all Sigma-Aldrich). After $30 \mathrm{~min}$, digestion supernatant containing cells was removed, filtered through a cell strainer, spun down and resuspended in $10 \mathrm{ml}$ of PBS containing $5 \%$ bovine serum albumin (BSA) and $5 \mathrm{mM}$ EDTA. Remaining tissue was then topped up with fresh digestion medium until no more cells were liberated.

Primary culture expansion and conditioned medium production. Primary stromal cell lines were expanded by plating the single-cell suspension of tissue digests onto plastic cell culture vessels and expanding the adherent fraction in RPMI 1640 (with 20\% fetal calf serum (FCS), antibiotics, 5 mM HEPES) (Sigma). Primary cell lines were used for assays between passage numbers 7 and 15. For the production of conditioned medium, sorted cell populations were plated at $1,000,000$ cells $\mathrm{ml}^{-1}$ in cell culture dishes with RPMI 1640 containing 5\% FCS (Life Technologies), antibiotics and $5 \mathrm{mM}$ HEPES for $16 \mathrm{~h}$. Next, supernatants were aspirated, spun down to remove cells and frozen at $-80^{\circ} \mathrm{C}$ until further use.

FACS and analysis. Single-cell suspensions obtained from tissue digests were blocked in PBS 5\% BSA containing human anti-Fc block (Miltenyi), except when CD16 was in the panel, stained for FACS analysis or sorting with Fixable Viability Dye eFluor 780 (eBioscience, 1:1,000), DAPI (Invitrogen, 1:50,000) and antibodies (all from Biolegend, except anti-Pdpn: clone NZ-1.3 from eBioscience and anti-FAP:sheep from Biotechne) in PBS with 5\% BSA and 5 mM EDTA for $20 \mathrm{~min}$ on ice. After washing in the same buffer, cells were either analyzed (LSRII or Fortessa X20) or sorted (Aria III, 100- $\mu$ m nozzle). All antibodies used for FACS analysis and sorting can be found in Supplementary Table 10.

Ex vivo and in vitro assays of fibroblast stimulation. For the stimulation of stromal cells, either Ccd18-Co colonic fibroblasts (ATCC, no. CRL-1459) or primary stromal cell lines (isolated as above) were plated at 20,000 cells per well in a 48 -well plate. Plated cells were starved for $72 \mathrm{~h}$ in culture medium without FCS, before stimulation with cytokines or conditioned medium (prediluted 1:3 in starving medium) for $3 \mathrm{~h}$ or $24 \mathrm{~h}$ at $37^{\circ} \mathrm{C}$. For blockade experiments, recombinant cytokines in starving or conditioned medium were preincubated with $2 \mathrm{mg} \mathrm{ml}^{-1}$ Anakinra (Kineret) or Adalimumab (Humira) for $1 \mathrm{~h}$ at room temperature, with shaking, before stimulation of cells. After $3 \mathrm{~h}$, supernatants were removed and cells lysed directly in appropriate RNA lysis buffer.
Isolation of RNA from tissue samples and cell populations. Endoscopic punch biopsies or dissected tissue pieces from surgical resections were stored in RNAlater (Qiagen) following collection, until further processing. Tissue was homogenized using the soft tissue homogenizing CK14 kit (Precellys, Stretton Scientific, no. 03961 ) in $300 \mu \mathrm{l}$ of RLT lysis buffer (Qiagen) and $20 \mu \mathrm{M}$ DTT (Sigma). RNA was isolated using the Qiagen Mini kit with a DNA digestion step (Qiagen). Bulk-sorted cell populations and cultured cells were directly lysed in RNA lysis buffer, followed by RNA isolation with the appropriate kits and on-column DNase treatment.

Quantitative real-time PCR. Normalized amounts of isolated RNA were reverse transcribed using a high-capacity reverse transcription kit (Thermo Scientific, no. 4368814). Quantitative real-time PCR (qPCR) was performed using premade, exon-spanning Taqman probes (LifeTechnologies) and run on a ViiA 7 Real-Time PCR system. Gene expression values, relative to the housekeeping gene(s) as indicated, were calculated using the $2^{\Delta \mathrm{ct}}$ method.

Sequencing of RNA from whole tissue and sorted cell populations. Sequencing libraries were prepared using either the QuantSeq 3' mRNA-Seq FWD Library Prep Kit (Lexogen) for whole-tissue samples, or the Smart-seq 2 protocol ${ }^{37}$ for bulk and cultured cell populations (with our own in-house indexing primers). Libraries were sequenced using an Illumina HiSeq4000 with 75-base-paired-end sequencing ${ }^{38}$. For qPCR analysis, 15-250 ng of RNA was reverse transcribed using the High-Capacity cDNA Reverse Transcription Kit (Applied Biosystems) and qPCR performed using Precision Fast qPCR mastermix with the EagleTaq Universal Master Mix at a lower level, $12.8 \mathrm{ml}$ (Primer design, Precision FAST-LR), and Taqman probes (Life Technologies).

Bulk RNA sequencing data were analyzed using the bulk processing aspect of pipeline_scrnaseq.py (https://github.com/sansomlab/scseq). Data quality was assessed using pipeline_readqc.py (https://github.com/cgat-developers/cgat-flow). Sequenced reads were aligned to the human genome GRCh38 with Hisat2 (v.2.1.0) $)^{39}$ using a reference index built from the GRCm38 release of the mouse genome and known splice sites extracted from Ensembl v.91 annotations (using the hisat2_extract_splice_sites.py tool). A two-pass mapping strategy was used to discover new splice sites (with these additional parameters: -dta and-score-min L,0.0,-0.2). Mapped reads were counted using featureCounts (Subread v.1.6.3; Ensembl v.91 annotations; with default parameters ${ }^{40}$. Salmon v.0.9.1 was used to calculate transcripts per million (TPM) values ${ }^{41}$ using a quasi-index (built with Ensembl v.91 annotations and $k=31$ ) and gc bias correction (parameter ' - gcBias'). For heatmap visualization of gene expression levels, $z$-scores of TPM values and Manhattan distances were calculated within the heatmap2 package in $\mathrm{R}$ Differential expression analyses were performed using DESeq2 (v.1.26.0 ${ }^{42}$.

Pathway enrichment analysis for groups of genes associated with cell types was carried out with the enrich $G O$ function from the clusterProfiler package in $\mathrm{R}^{43}$. 'Cellular component' Gene Ontology annotation terms were used as pathways.

Identification and quantification of gene coexpression modules in discovery data. To reduce dimensionality within the dataset, an unbiased approach was used to collapse genes with similar expression patterns in the discovery RNA-seq dataset. Normalized (TPM) counts were considered for all genes across all samples, including both inflamed and noninflamed tissues from patients with IBD and samples from the CRC controls. These were filtered to remove genes with zero counts in over half of the samples, and log transformed following the addition of a pseudocount. Transformed counts were then used to define modules of correlated genes using WGCNA in $\mathrm{R}^{44}$. In brief, this process calculates pairwise Pearson correlation estimates between all genes; these are then raised to the power of a soft threshold, in this case raising correlation coefficients to the power of 9 , which magnifies the differences between large and small correlations. Finally, the network of these amplified correlations (where each gene is a node and each edge is a correlation) is used to generate a topological overlap matrix (TOM). This represents the similarity of expression patterns between a given pair of genes in the dataset, similar to the correlation matrix but taking into account their shared correlation with other genes. Finally, hierarchical clustering of the TOM is used to assign genes into modules based on their coexpression pattern The pickSoft Threshold function was used to identify nine as an appropriate soft threshold. The blockwiseModules function was then used with this threshold to automatically carry out the aforementioned process and assign genes to modules. Parameters for the function were as follows: minimum module size of 30 genes, mergeCutHeight of 0.1 , reassignThreshold of 0 and use of a signed network.

The resultant module definitions were quantified using the eigengene approach within WGCNA. An eigengene is a quantitative representation of the expression of a module as a whole, and is derived from the first component of a principle components analysis restricted to the expression data of only genes in the module. Eigengenes for the modules defined in the resection data were calculated using the moduleEigengenes function.

Correlations between clinical and metadata measures and module eigengenes were assessed using Pearson correlations, with $P$ values estimated using the corPvalueStudent function and adjusted for multiple testing. Benjamini-Hochberg correction using the p.adjust function was used for all analyses with adjusted 
$P$ values. This was carried out on inflamed IBD tissue samples and CRC tissue samples combined, and also on inflamed IBD tissue samples alone. Eigengenes were also compared between paired inflamed and noninflamed tissues sections using a $t$-test and adjusting for multiple testing across modules.

Cell type composition scores were estimated for each resection sample using the $x$ CellAnalysis function from the $\mathrm{xCell}$ package ${ }^{14}$. Correlations between module eigengenes and the derived cell type scores were visualized for all cell types scored in $>25 \%$ of samples, and used to infer the cell types represented by modules within the whole-tissue data (discovery cohort).

Quantification of module associations with clinical variables in replication datasets. Publicly available RNA-seq ${ }^{10,17}$ (GSE57945, GSE109142) or microarray data $^{15,16,36}$ (GSE16879, GSE12251, GSE100833) were downloaded from the NCBI gene expression omnibus. These were pre-existing enumerated gene counts in the case of the RNA-seq datasets and raw array data in the case of the microarray sets. The latter were processed and normalized to gene counts using the rma function from the affy package ${ }^{45}$, summing values for probes associated with the same gene symbol. Across all datasets, gene symbol annotations were used to map genes to the module assignments generated from the discovery resection tissue dataset, eliminating those not observed in the replication dataset under consideration. The percentage of genes missing from the original module definitions was recorded, but was generally low across all datasets. Mapped module assignments were then used to generate eigengenes from the replication expression datasets using the moduleEigengenes function. Correlations between clinical metadata and eigengenes in replication datasets was performed using Pearson correlations as for the discovery dataset. In the case of the pediatric cohort data (GSE57945), Mann-Whitney $U$-tests were used to compare modules between patients scored as ulcerated or not in metadata, and hierarchical clustering was used to group patients based on M4, M5 and M6 expression as for the discovery cohort.

Differences in pretreatment module eigengene values between responders and nonresponders in prospective studies were assessed using Mann-Whitney $U$-tests, adjusting $P$ values for testing of multiple modules within each dataset. In the study of Haberman et al. ${ }^{10}$, we considered only patients on corticosteroid therapy, combining patients that received oral and intravenous administration. In the case of the 2018 study of Arjis et al. ${ }^{16}$, which tested multiple different therapies and treatment regimens, we used analysis of variance (ANOVA) to identify any differences between responders and nonresponders across all combinations, adjusting for regimen, and post hoc Mann-Whitney $U$-tests to identify individual treatment regimens where modules were significantly different by response.

Meta-analysis of the expression of modules M4 and M5 across responders and nonresponders in the various replication datasets was carried out using the meta package in $\mathrm{R}^{46}$. Anti-TNF response data were used from the Arijs $2009^{15}$ and $2018^{16}$ papers, and corticosteroid response data were from the study of Haberman et al. Only the 4-week treatment condition was included from the anti-intergrin data from ref. ${ }^{16}$, as this was the only one that proved significantly different for either M4 or M5. A random effects meta-analysis was carried out comparing standardized mean differences between patient groups using the exact Hedges estimate.

In the prospective cohorts, the predictive value of the expression of single genes for response to treatment was assessed using a simple logistic regression where response was the outcome and gene expression the sole predictor. Modeling was carried out for all genes also observed in the discovery cohort, for each of the prospective studies, using the $\mathrm{glm}$ function in R. The predictive ability of each gene in each dataset was summarized as the area under the curve (AUC) of a receiver operating characteristic (ROC) curve. AUC values for each gene were generated by applying the roc function from the pROC package to predictions generated from the logistic regression models. The relative predictive power of genes within modules of interest was compared by summing the rank of genes (based on their AUC value) across datasets and comparing these cumulative ranks between modules.

Pathological scoring of histology using the Nancy index. Formalin-fixed paraffin-embedded (FFPE) and hematoxylin-and-eosin-stained tissue sections of IBD patients were scored according to the Nancy index, based on criteria reported in ref. ${ }^{13}$. The extent of histologic ulceration was scored on a semiquantitative scale $(0-25-50-75-100 \%$ of section area). All scorings were carried out by blinded, consultant gastrointestinal histopathologists.

Immunohistochemistry and quantitative histopathology. Tissue specimens were either fixed for $48 \mathrm{~h}$ in $4 \%$ neutral-buffered formalin (Sigma) and embedded in paraffin (FFPE) for chromogenic stainings, or fixed for $24 \mathrm{~h}$ in $2 \%$ paraformaldehyde in phosphate buffer containing L-lysine and sodium periodate and frozen in OCT (Sigma) after soaking in 30\% sucrose for $48 \mathrm{~h}$ (OCT) for fluorescent staining. Freshly cut, dewaxed and rehydrated FFPE sections $(5 \mu \mathrm{m})$ were subjected to heat-induced antigen retrieval by boiling in Target Retrieval Solution (Dako, pH 6.0, for all stainings except neutrophil elastase) for $15 \mathrm{~min}$ (microwave). This was followed by $15 \mathrm{~min}$ of blocking in Bloxall solution (Vector Labs), 60 min of blocking in 5\% BSA/TBST with 5\% serum of secondary antibody species (Sigma) and $15 \mathrm{~min}$ of blocking in avidin followed by biotin solution (Vector Labs). All steps were performed at ambient temperature. Tissue sections were incubated with primary antibodies in 5\% BSA/TBST overnight $(>16 \mathrm{~h})$ at
$4{ }^{\circ} \mathrm{C}$. Following incubation, biotinylated or HRP-conjugated secondary antibodies were applied for $2 \mathrm{~h}$ (room temperature) in 5\% BSA/TBST. For biotinylated secondary antibodies, $\mathrm{AB}$ complex (Vector Labs) was incubated for a further $1 \mathrm{~h}$ in TBST (room temperature). Chromogenic stains were developed by the application of DAB HRP substrate solution (Vector Labs) and counterstained for $5 \mathrm{~min}$ in hematoxylin solution (Sigma). Slides were then dehydrated and mounted in DPX (Sigma) mounting medium.

Whole-section imaging of chromogenic sections was performed on a NanoZoomer S210 digital slide scanner (Hamamatsu). Slide scans of all stains can be made available upon request. Scanned tissue sections, stained using DAB immunohistochemistry, were analyzed using Indica Labs HALO image analysis platform. A consultant gastrointestinal pathologist manually annotated each slide, dividing the mucosa into normal and inflamed areas. The tissue was scored using Indica Labs' analysis modules CytoNuclear v.2.0.5, detecting DAB-positive and -negative cells in inflamed areas. Pathologic features (ulceration/granulation tissue, granulomas, crypt abscess/cryptitis, lymphoid aggregates and architectural distortion/ mucin depletion) were manually annotated by a consultant pathologist with a special interest in gastrointestinal pathology. The area of each annotated feature was automatically calculated using HALO software. Nuclei (cells) in areas of interest and whole-tissue sections were detected and counted using Indica Labs' CytoNuclear v.2.0.9 analysis module. Scores (percentage) were normalized to the number of nuclei found within a pathological feature over the total number of nuclei detected in the whole-tissue section. These normalized counts were used to investigate Pearson correlations between features and correlations with module eigengenes.

OCT sections ( $10 \mu \mathrm{M}$ thickness) were incubated in blocking buffer (PBS1X, 5\% goat serum, 2\% FCS and human FcBlock, Miltenyi) with unconjugated primary antibodies (PDPN, PDGFRa, ABCA8), conjugated primary antibodies (THY1, NE, PECAM1, MCAM) or biotinylated (FAP) overnight at $4{ }^{\circ} \mathrm{C}$. The following day, either AF488 donkey anti-rat, AF647 donkey anti-goat, AF555 donkey anti-rabbit or strepatividin-AF568 was applied for $1 \mathrm{~h}$ at room temperature in blocking buffer. Finally, nuclei were stained with Hoechst 28332 (Life Technologies) for $15 \mathrm{~min}$ at room temperature in blocking buffer and mounted in ProlongGold mounting medium (Life Technologies) before imaging with the spectral detector of a Zeiss confocal LSM 880 microscope. Images were processed and converted to TIFF format in Image J. All antibodies used for immunohistochemistry can be found in Supplementary Table 10

Preparation of cells for scRNA-seq. Four pairs of biopsies from the same patient were pooled, minced and frozen in $1 \mathrm{ml}$ of CryoStor CS10 (StemCell Technologies) at $-80^{\circ} \mathrm{C}$, then transferred to liquid nitrogen within $24 \mathrm{~h}$. Single-cell suspensions from these endoscopic biopsies were then prepared by thawing, washing and subsequent mincing of the tissue using surgical scissors. Minced tissue was then subjected to rounds of digestion in RPM 1640 medium (Sigma) containing 5\% FBS (Life Technologies), $5 \mathrm{mM}$ HEPES (Sigma), antibiotics as above and Liberase TL with DNAse I (Sigma). After $30 \mathrm{~min}$, digestion supernatant was removed, filtered through a cell strainer, spun down and resuspended in $10 \mathrm{ml}$ of PBS containing $5 \%$ BSA and $5 \mathrm{mM}$ EDTA. Remaining tissue was then topped up with fresh digestion medium until no further cells were liberated from the tissue. Cells were then stained and FACS-sorted, as described above for live $\mathrm{EPCAM}^{-} \mathrm{CD}_{4} 5^{-}$cells, before being taken for microfluidic partitioning.

\section{X library preparation, sequencing and data analysis. Single-cell RNA-seq} data was generated from disaggregated intestinal tissue sorted for CD45-EPCAM ${ }^{-}$ stromal cells. Viable cells were subjected to a standard droplet single-cell complementary DNA library preparation protocol. The experimental details for generation of cDNA libraries are described in a separate manuscript ${ }^{47}$. We demultiplexed FASTQ files for each 10X library using the Cell Ranger (v.3.1.0) mkfastq function ${ }^{48}$. We then mapped reads to the GRCh38 human genome reference using Kallisto ${ }^{49}$ (v.0.46.0) and quantified genes by cell-barcode UMI matrices with Bustools (https://github.com/BUStools/bustools) (v.0.39.0). For quantification we used gene annotations provided by Gencode ${ }^{50}$ (release 33), keeping only protein-coding genes and collapsing Ensembl transcripts to unique HGNC-approved gene symbols.

We filtered for potentially empty droplets and damaged cells by excluding droplets with $<500$ unique genes and libraries with $>20 \%$ of reads assigned to mitochondrial genes. We pooled the resulting high-quality cells from each $10 \mathrm{X}$ library into a single cell by gene UMI matrix. We normalized for read depth with the standard $\log (\mathrm{CP} 10 \mathrm{~K})$ normalization procedure for gene $g$ and cell $i$, where log $\mathrm{CP} 10 \mathrm{~K}_{g i}=$ normalized log counts-per-10,000 for gene $g$ and cell $i, h=$ index for gene $h$, and $\Sigma_{h} \mathrm{UMI}_{h i}=$ total number ofcounts observed for cell $i$ :

$$
\log \mathrm{CP} 10 K_{g i}=\log \left(1+10^{4} \times \frac{\mathrm{UMI}_{g i}}{\sum_{h} \mathrm{UMI}_{h i}}\right)
$$

We performed PCA analysis on the top 2,000 most variable genes, identified with the VST method implemented in the Seurat ${ }^{51}$ R package. For PCA, we $z$-scored each variable gene and computed the top 30 eigenvectors and singular values with the truncated SVD procedure, implemented in the RSpectra (https:// github.com/yixuan/RSpectra) R package. We defined PCA cell embeddings by 
scaling eigenvectors by their respective singular values. To account for potential batch effects in PCA embeddings, we modeled and removed the effect of the $10 \mathrm{X}$ library as identified using the Harmony algorithm. For Harmony ${ }^{19}$, we set the cluster diversity penalty parameter $\theta$ to 0.5 and used default values for all other parameters. We evaluated the effect of library mixing before and after Harmony using the local inverse Simpson's index (LISI), described in the Harmony manuscript ${ }^{19}$. We evaluated the significance of change in LISI with a $t$-test, with degrees of freedom equal to the number of libraries minus 1 . To visualize cells in two dimensions, we input the Harmonized PCs into the uniform manifold approximation and projection (UMAP) (arXiv:1802:03426 [stat.ML]) algorithm.

Identification of marker genes within scRNA-seq. We performed joint clustering analysis on all scRNA-seq libraries using the cells' harmonized PCA embeddings. With the 30-nearest-neighbor graph, we computed the unweighted shared nearest neighbor (SNN) graph and truncated SNN similarity values $<1 / 15$ to zero. We then performed Louvain clustering based on the $\mathrm{R} / \mathrm{C}++$ implementation from Seurat at resolution 0.3 , resulting in eight clusters. We identified upregulated marker genes in each cluster using pseudobulk differential expression with negative binomial regression, implemented in the DESeq2 R package. For pseudobulk analysis, we collapsed cells from the same donor and cluster into one pseudobulk sample, summing the UMI counts from each cell. We then performed differential expression analysis on these pseudobulk samples, with the design $y \sim 1+$ cluster. This design assigns each gene an intercept term (that is, mean expression), a multiplicative offset for each cluster. We addressed the degeneracy of the design matrix by assigning a Gaussian prior distribution to the cluster effects (DESeq2 parameter $\beta$ Prior=TRUE). The full results for this differential expression analysis are reported in Supplementary Table 6.

Differential expression analysis of single-cell data by inflammatory status. We performed differential expression to associate genes with inflammation status within each single-cell cluster. We used DESeq2 on the pseudobulk samples described above, this time analyzing each cluster separately with the design $y \sim 1+$ InflamStatus. We treated InflamStatus as a random effect (DESeq2 parameter $\beta$ Prior=TRUE) and recovered a mean multiplicative offset for each of the three inflammatory status categories.

Single-cell gene-set enrichment scoring. Single-cell gene-set enrichment scores were computed for WGCNA modules and cytokine stimulation signatures using the same strategy. For each gene in the gene set, we computed $z$-scores (mean centered and unit variance scaled) of $\log (\mathrm{CP} 10 \mathrm{~K})$ normalized expression across all cells. We then summed the $z$-scores of genes in the gene set to compute a single gene-set score for each cell. This procedure is summarized in the formula below, used to compute the score $S_{G, i}$ for gene set $G$ and cell $i$ using normalized expression $y_{g i}$, gene mean $\mu$, and gene standard deviation $\sigma_{g}$ :

$$
\text { score }_{G, i}=\sum_{g \in G}\left(y_{g i}-\mu_{g}\right) / \sigma_{g}
$$

Single-cell trajectory analysis. We performed trajectory analysis using the principal curve method, implemented in the princurve R package (https://www.jstor.org/ stable/2289936). We fit a principal curve to all fibroblasts by inputting harmonized UMAP coordinates into the principal_curve function. This mapped fibroblasts to a nonlinear, one-dimensional space and assigned each cell a unique position, from 0 to 100 , along this trajectory. For direct visualization of the abundance of each cluster along the trajectory, we plotted the relative density of each cluster along it. In these density plots, $\mathrm{ABCA} 8^{+}$fibroblasts grouped towards the beginning (position 32 ) of the trajectory, $\mathrm{PDPN}^{+}$fibroblasts in the middle (position 59) and PDGFRA ${ }^{+}$ fibroblasts towards the end (position 82). This distribution along the trajectory is also reflected by the canonical markers of these populations. To visualize this, we discretized pseudotime by binning into 100 uniform-density windows, chosen so that each window has the same number of cells. We then plotted the scaled gene expression values of ABCA8, PDPN and PDGFRA, summarized by mean expression (point) and 95\% confidence interval (line).

Reporting Summary. Further information on research design is available in the Nature Research Reporting Summary linked to this article.

\section{Data availability}

Bulk RNA sequencing has been deposited at GEO (GSE166928) and single-cell data at ImmPort (SDY1765; accessible with the next release scheduled for 10 September 2021). Additional data have been made available through a GitHub repository at https://github.com/microbialman/IBDTherapyResponsePaper. Due to their extensive size, image scans and raw data for FACS/qPCR assays are available from the corresponding author (F.M.P.) upon request. Publicly available RNA-seq (GSE57945, GSE109142) and microarray data (GSE16879, GSE12251, GSE100833) were downloaded from the NCBI gene expression omnibus. Publicly available RNA-seq (GSE57945, GSE109142) and microarray data (GSE16879, GSE12251, GSE100833) were downloaded from the NCBI gene expression omnibus.

\section{Code availability}

All code used for analysis and generation of figures can be found in the GitHub repository at https://github.com/microbialman/IBDTherapyResponsePaper.

\section{References}

36. Peters, L. A. et al. A functional genomics predictive network model identifies regulators of inflammatory bowel disease. Nat. Genet. 49, 1437-1449 (2017).

37. Picelli, S. et al. Smart-seq 2 for sensitive full-length transcriptome profiling in single cells. Nat. Methods 10, 1096-1098 (2013).

38. Lamble, S. et al. Improved workflows for high throughput library preparation using the transposome-based Nextera system. BMC Biotechnol. 13, 104 (2013).

39. Kim, D., Langmead, B. \& Salzberg, S. L. HISAT: a fast spliced aligner with low memory requirements. Nat. Methods 12, 357-360 (2015).

40. Liao, Y., Smyth, G. K. \& Shi, W. featureCounts: an efficient general purpose program for assigning sequence reads to genomic features. Bioinformatics $\mathbf{3 0}$, 923-930 (2014)

41. Patro, R., Duggal, G., Love, M. I., Irizarry, R. A. \& Kingsford, C. Salmon provides fast and bias-aware quantification of transcript expression. Nat. Methods 14, 417-419 (2017).

42. Love, M. I., Huber, W. \& Anders, S. Moderated estimation of fold change and dispersion for RNA-seq data with DESeq2. Genome Biol. 15, 550 (2014).

43. Yu, G., Wang, L. G., Han, Y. \& He, Q. Y. clusterProfiler: an R package for comparing biological themes among gene clusters. OMICS 16, 284-287 (2012)

44. Langfelder, P. \& Horvath, S. WGCNA: an R package for weighted correlation network analysis. BMC Bioinformatics 9, 559 (2008).

45. Gautier, L., Cope, L., Bolstad, B. M. \& Irizarry, R. A. affy-analysis of Affymetrix GeneChip data at the probe level. Bioinformatics 20, 307-315 (2004).

46. Balduzzi, S., Rucker, G. \& Schwarzer, G. How to perform a meta-analysis with R: a practical tutorial. Evid. Based Ment. Health 22, 153-160 (2019).

47. Korsunsky, I. et al. Cross-tissue, single-cell stromal atlas identifies shared pathological fibroblast phenotypes in four chronic inflammatory diseases. Preprint at https://www.biorxiv.org/content/10.1101/2021.01.11.426253v1 (2021)

48. Zheng, G. X. et al. Massively parallel digital transcriptional profiling of single cells. Nat. Commun. 8, 14049 (2017).

49. Bray, N. L., Pimentel, H., Melsted, P. \& Pachter, L. Near-optimal probabilistic RNA-seq quantification. Nat. Biotechnol. 34, 525-527 (2016).

50. Harrow, J. et al. GENCODE: the reference human genome annotation for The ENCODE Project. Genome Res. 22, 1760-1774 (2012).

51. Stuart, T. et al. Comprehensive integration of single-cell data. Cell 177, 1888-1902 (2019).

\section{Acknowledgements}

M.F. was supported by a Roche postdoctoral fellowship (2016-2017) and the Oxford-UCB-prize fellowship scheme (2017-2021). M.P. was supported by the Roche postdoctoral fellowship program (2017-2019). M.A.J. was funded by the Kennedy Trust for Rheumatology Research (no. MSP141501) and supported by a Junior Research Fellowship from Linacre College Oxford. We acknowledge the contribution of the Oxford Biomedical Research Centre (BRC) Gastrointestinal Biobank (TGU Biobank Consortium: H.H.U., S.P.T., F.M.P.) supported by the National Institute for Health Research (NIHR) BRC. We thank members of the Oxford TGU biobank, especially J. Chivenga, A. Isherwood, R. Williams and G. Zavalani for facilitating the collection of patient samples. This work was supported by the NIHR BRC. The views expressed are those of the author(s) and not necessarily those of the NHS, the NIHR or the Department of Health. F.M.P. and H.H.U. are supported by the Leona M. and Harry B. Helmsley Charitable Trust. Parts of this work were also supported by Roche Fibroblast Network Consortium funding, NIHR OxBRC mucosal immunology response mode funding, the Wellcome Trust (nos. 095688/Z/11/Z and 212240/Z/18/Z), the Medical Research Council (no. MR/N02690X/1) and the Translational Histopathology Laboratory at the Oxford Cancer Centre. We further thank J. Pott for helping to set up ex vivo functional assays and the following Kennedy Institute core facility members for excellent technical support and training: J. Webber, B. Stott, I. Parisi, C. dos Santos Duarte and V. Nechyporuk-Zloy.

\section{Author contributions}

Conceptualization was the responsibility of M.F., M.P., M.A.J. and F.M.P. Methodology was undertaken by M.F., M.P., M.A.J., I.K., S.J.B., K.R.-A., D.S., M.A., K.W., G.F.M.W., Roche Fibroblast Network Consortium, A.E., S.S., S.R., S.P.T. and F.M.P. Software was provided by M.A.J. and I.K. Validation was performed by M.F., M.P., M.A.J. and I.K. Formal analysis was undertaken by M.F., M.P., M.A.J., I.K., K.R.-A., E.C. and A.E. M.F., M.P., S.J.B., D.S., H.S., R.R., R.T. and A.G. performed investigations. Resources were the responsibility of M.F., M.P., Z.C., R.R., R.S.P., E.H.M., A.G., T.T., Oxford IBD Cohort Investigators, Roche Fibroblast Network Consortium, S.M., L.T., S.S., S.R., S.P.T. and F.M.P. Data curation was performed by M.F., M.P., M.A.J., I.K., S.J.B., K.R.-A., Z.C., D.S., R.R., L.T. and A.E. Writing of the original draft was done by M.F., M.P., M.A.J. and 
F.M.P. Writing, review and editing were done by all authors listed. Visualization was performed by M.F., M.P., M.A.J., I.K. and K.R.-A. M.F., M.P., S.J.B., S.S., S.R., S.P.T. and F.M.P. supervised the project. Project administration was undertaken by M.F., M.P., TGU Biobank Consortium, S.P.T. and F.M.P. Funding acquisition was overseen by M.F., I.K., S.J.B., Z.C., S.R., S.P.T. and F.M.P.

\section{Competing interests}

F.M.P. received research support from Roche and Janssen, and consulting fees from GSK, Novartis and Genentech. H.H.U. received research support or consultancy fees from Janssen, Eli Lilly, UCB Pharma, BMS/Celgene, MiroBio, Mestag and OMass. S.P.T. received research support from AbbVie, Buhlmann, Celgene, IOIBD, Janssen, Lilly, Pfizer, Takeda, UCB, Vifor and the Norman Collisson Foundation; consulting fees from AbbVie, Allergan, Abiomics, Amgen, Arena, Asahi, Astellas, Biocare, Biogen, Boehringer Ingelheim, Bristol-Myers Squibb, Buhlmann, Celgene, Chemocentryx, Cosmo, Enterome, Ferring, Giuliani SpA, GSK, Genentech, Immunocore,

Immunometabolism, Indigo, Janssen, Lexicon, Lilly, Merck, MSD, Neovacs, Novartis, NovoNordisk, NPS Pharmaceuticals, Pfizer, Proximagen, Receptos, Roche, Sensyne,
Shire, Sigmoid Pharma, SynDermix, Takeda, Theravance, Tillotts, Topivert, UCB, VHsquared, Vifor and Zeria; and speaker fees from AbbVie, Amgen, Biogen, Ferring, Janssen, Pfizer, Shire, Takeda and UCB (no stocks or share options). M.A.J. is an employee of MiroBio Ltd. K.G.L. and A.P.F. are employees of Roche Ltd. All other authors declare no competing interests.

\section{Additional information}

Extended data is available for this paper at https://doi.org/10.1038/s41591-021-01520-5. Supplementary information The online version contains supplementary material available at https://doi.org/10.1038/s41591-021-01520-5.

Correspondence and requests for materials should be addressed to Fiona M. Powrie. Peer review information Nature Medicine thanks Arthur Kaser and the other, anonymous, reviewer(s) for their contribution to the peer review of this work. Primary Handling editor: Saheli Sadanand, in collaboration with the Nature Medicine team.

Reprints and permissions information is available at www.nature.com/reprints. 


\begin{tabular}{|c|c|c|c|c|}
\hline Characteristic & $\begin{array}{l}\text { non-IBD } \\
(n=39)\end{array}$ & $\begin{array}{l}\text { IBD } \\
(\mathrm{UC}+\mathrm{CD}+\text { IBDu) } \\
(\mathrm{n}=31)\end{array}$ & $\begin{array}{l}U C \\
(n=8)\end{array}$ & \begin{tabular}{|l|}
$C D$ \\
$(n=22)$
\end{tabular} \\
\hline$\%$ Male (Male/Female) & $54(21 / 18)$ & $52(16 / 15)$ & $60(3 / 5)$ & $59(13 / 9)$ \\
\hline $\begin{array}{l}\text { Median (IQR) age at } \\
\text { sampling (years) }\end{array}$ & $68(60-76)$ & 37 (29-49) & $36(35-52)$ & $42(29-54)$ \\
\hline $\begin{array}{l}\text { Median (IQR) disease } \\
\text { duration at sampling } \\
\text { (years) }\end{array}$ & $n / a$ & $10(5-20)$ & $5(5-20)$ & $10(5-20)$ \\
\hline $\begin{array}{r}\text { *Sampling site }(\%) \\
\text { Large intestine } \\
\text { Small intestine }\end{array}$ & $\begin{array}{l}39(100) \\
0(0)\end{array}$ & $\begin{array}{l}27(66) \\
14(34)\end{array}$ & $\begin{array}{l}10(100) \\
0(0)\end{array}$ & $\begin{array}{l}16(53) \\
14(47)\end{array}$ \\
\hline $\begin{array}{l}\text { Medication (ever) before } \\
\text { surgery (\%) } \\
\text { Aminosalicylates } \\
\text { Corticosteroids } \\
\text { Immunomodulator } \\
\text { (e.g. Azathioprine) } \\
\text { Anti-TNF } \\
\text { (Infliximab or } \\
\text { Adalimumab) } \\
\text { Anti-Integrin } \\
\text { (Vedolizumab) } \\
\text { Monotherapy } \\
\text { (one of the above) } \\
\text { Combination therapy } \\
\text { (two or more of the } \\
\text { above) } \\
\text { Treatment-naïve } \\
\text { (none of the above) }\end{array}$ & $\begin{array}{l}n / a \\
n / a \\
n / a \\
n / a \\
n / a \\
n / a \\
n / a \\
n / a\end{array}$ & $\begin{array}{l}6(19) \\
12(39) \\
21(68) \\
13(42) \\
2(7) \\
10(32) \\
15(48) \\
6(20)\end{array}$ & $\begin{array}{l}5(63) \\
3(38) \\
2(25) \\
1(6) \\
1(6) \\
4(50) \\
3(38) \\
1(12)\end{array}$ & \begin{tabular}{|l}
$1(5)$ \\
$9(41)$ \\
$11(50)$ \\
$7(32)$ \\
$1(5)$ \\
$6(27)$ \\
$12(55)$ \\
$4(18)$
\end{tabular} \\
\hline
\end{tabular}

Extended Data Fig. 1 | Clinical characteristics of the Oxford IBD patient discovery cohort used in this study. Samples from the discovery cohort consist of surgically removed tissue of UC, CD or IBDu patients (=IBD), as well as surgically removed normal tissue adjacent to colorectal tumors (=non-IBD). IBD, inflammatory bowel disease; CD, Crohn's Disease; UC, Ulcerative colitis; IQR, interquartile range; n/a, not applicable. * Sampling site percentages are based on the total number of tissues collected (not patient number). 
a

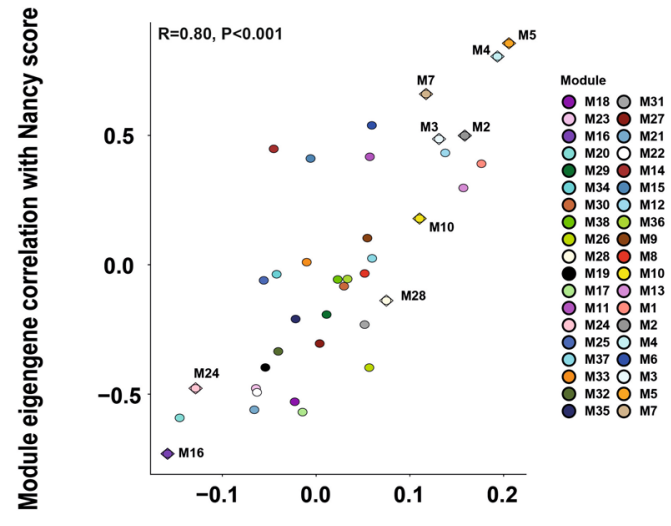

Mean difference module expression (inflamed/uninflamed)

b

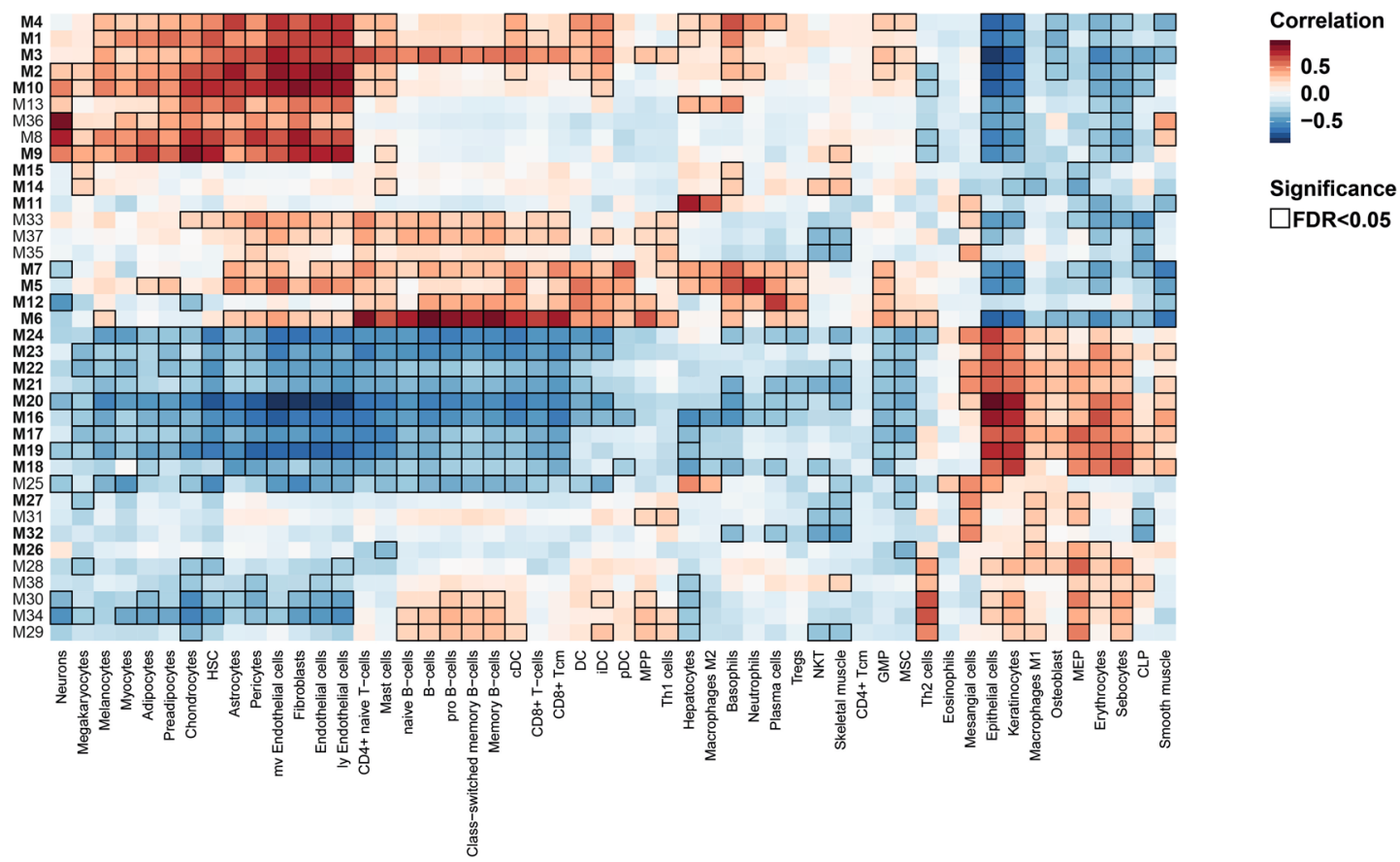

C

d

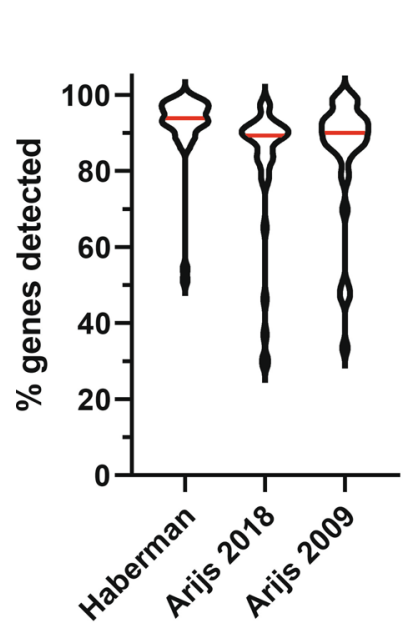

e

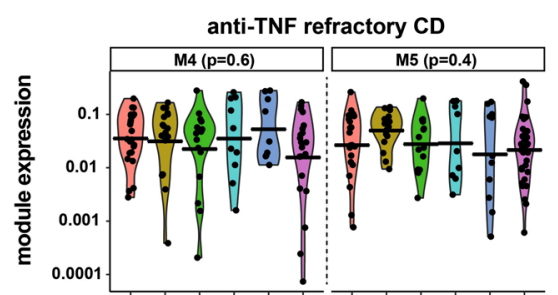

Sampling site

$\square$ Rectum

$\square$ Sigmoid colon

$\square$ Descending colon

$\square$ Transverse colon

$\square$ Ascending colon

$\square$ Terminal ileum

Extended Data Fig. 2 | See next page for caption. 
Extended Data Fig. 2 | Module associations with inflammation and response to therapy. a) Scatterplot of the module expression difference between inflamed and uninflamed tissues paired from the same patients versus the correlation of the module with the Nancy score across all IBD and non-IBD tissues. Points highlighted with a diamond indicate a significant difference in two-tailed paired t-tests between inflamed/uninflamed tissue (FDR $p<0.1$ ). b) Heatmap of module eigengene - cell type correlations; cell types were deconvoluted from whole tissue expression data using $x$ Cell. Modules highlighted in bold were fund to be associated with histologic inflammation. c) Percentage of genes within each of the $n=38$ modules that were detectable in the publicly available datasets ${ }^{10,15,16}$. Red horizontals in violins indicate the median. $d$ ) M4/M5 module expression (eigengene) in patients with $C D(n=37)$ and UC $(n=24)$ before anti-TNF therapy start (GSE16879, horizontal bars indicate geometric mean, two-tailed Mann Whitney $U$ test $P$ values are given; exact $P$-values are 0.0026 and $7.8 \times 10^{-6}$ for M4 and M5 CD comparisons, respectively). e) M4/M5 module expression (eigengene) in $n=87$ patients with CD after anti-TNF therapy failure (GSE100833, horizontal bars indicate geometric mean, P values of comparisons across all sites are given, Kruskall-Wallis test). 


\begin{tabular}{|c|c|c|}
\hline Characteristic & $\begin{array}{l}\text { UC } \\
\text { responders } \\
\text { to anti-TNF } \\
(n=35)\end{array}$ & $\begin{array}{l}\text { UC non- } \\
\text { responders to } \\
\text { anti-TNF } \\
(n=23)\end{array}$ \\
\hline$\%$ Male (Male/Female) & $65.7(23 / 12)$ & $53.3(8 / 15)$ \\
\hline $\begin{array}{l}\text { Median (IQR) age at } \\
\text { sampling (years) }\end{array}$ & $32(23-51)$ & $34(24-52)$ \\
\hline $\begin{array}{l}\text { Median (IQR) disease } \\
\text { duration at sampling } \\
\text { (years) }\end{array}$ & $3(1-9)$ & $2(1-7)$ \\
\hline $\begin{array}{l}\text { Medication (ever) during } \\
\text { follow-up (\%) }\end{array}$ & & \\
\hline Aminosalicylates & $26(74.3)$ & $17(81.0)$ \\
\hline Corticosteroids & $18(51.4)$ & $16(76.2)$ \\
\hline $\begin{array}{l}\text { Immunomodulator } \\
\text { (e.g. Azathioprine) }\end{array}$ & $17(48.6)$ & $11(52.4)$ \\
\hline $\begin{array}{l}\text { Treatment-naïve } \\
\text { (none of the above) }\end{array}$ & $0(0)$ & $0(0)$ \\
\hline
\end{tabular}

Extended Data Fig. 3 | Clinical characteristics of the Oxford UC patient cohort of response to anti-TNF therapy. Response to therapy in this UC patient cohort was defined as stopping anti-TNF therapy (Infliximab or Adalimumab) within 12 months of start, for reason of non response (patients that stopped therapy for convenience, switch to biosimilar, intolerance, or anti-drug antibodies were not considered). Nancy histologic scores and UCEIS endoscopic scores, as well as the other characteristics, within 3 months before the start of anti-TNF therapy are shown. UC, Ulcerative colitis; IQR, interquartile range; UCEIS, Ulcerative Colitis Endoscopic Index of Severity. 
a

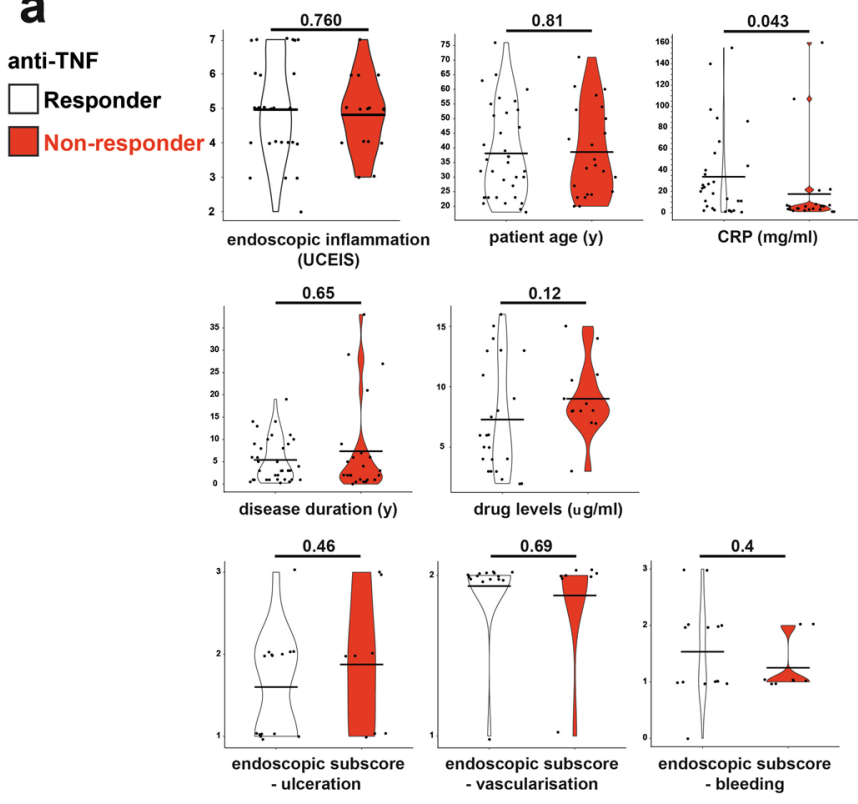

C

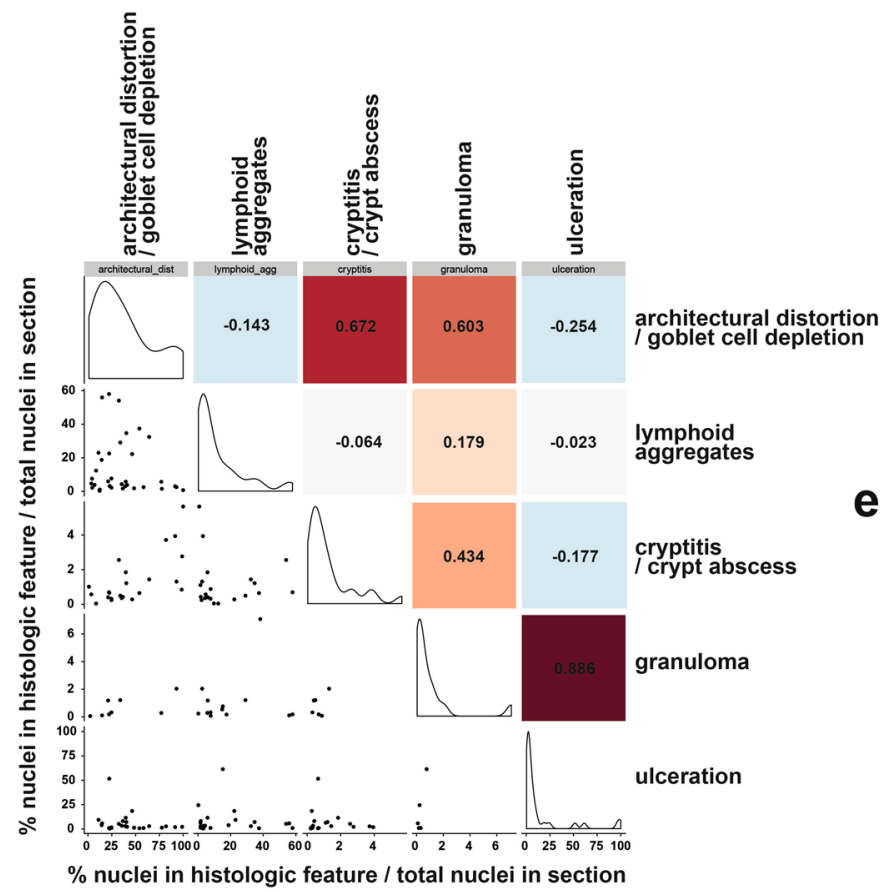

f

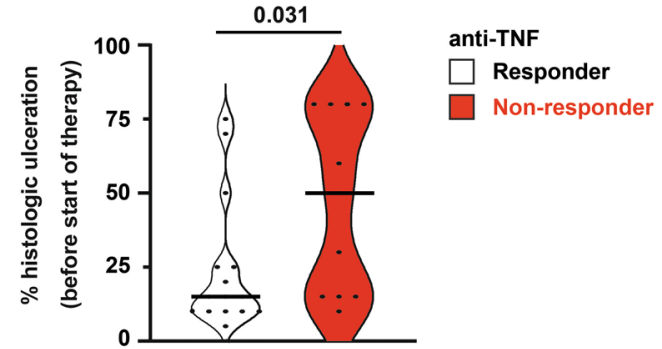

b

architectural distortion

I goblet cell depletion lymphoid aggregates

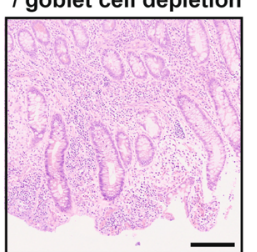

cryptitis

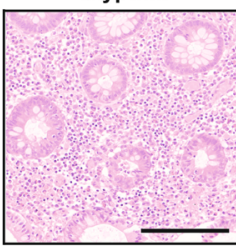

granuloma

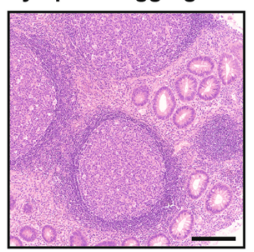

crypt abscess

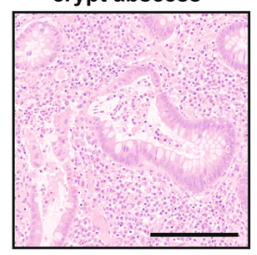

ulceration
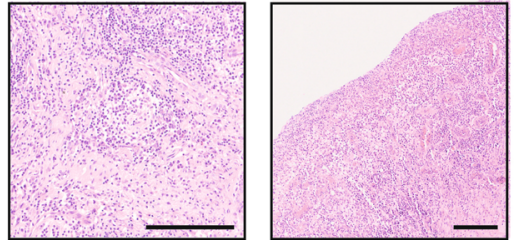

d

no ulceration

deep ulceration

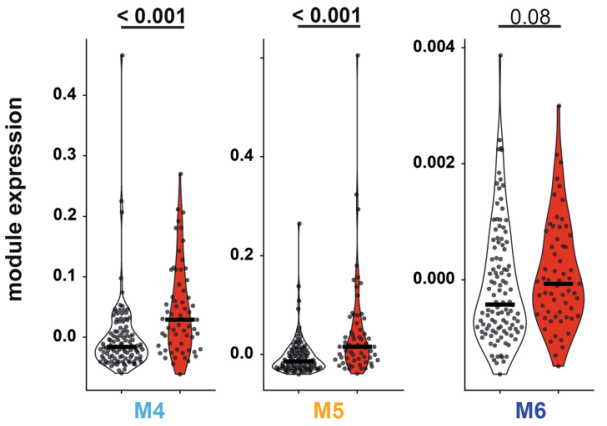

e

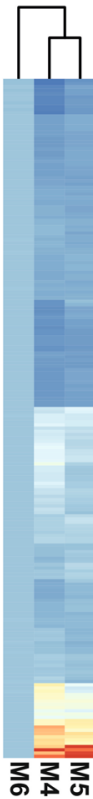

all low $(89 \%)$

M5/M4 high (11\%)

module

expression

0.3
0.25

0.2

0.2
0.15
0.1

0.05

0
-0.05

Extended Data Fig. 4 | See next page for caption. 
Extended Data Fig. 4 | Correlations of histopathologic measures and patient response to therapy. a) Clinical and endoscopic measures in responders $(n=35)$ and non-responders $(n=21)$ to anti-TNF therapy before the start of treatment (see Extended Data Fig. 3 for cohort details; horizontal bars indicate geometric mean, two-tailed Mann Whitney $U$ test $P$ values are given; some measures were not reported for all patients, resulting in less datapoints). b) Exemplary images of the various pathological features quantified on H\&E histology in resected tissue from IBD patients. Scale bar $200 \mu$ m. c) Correlation plot of histological features, quantified as the $\%$ of nuclei within the feature area relative to the nuclei with the total section area. Numbers and colours in upper right corner indicate the Pearson correlation coefficient; histograms on diagonal show the value distribution of the features within IBD patient tissues; scatter plots in the lower left corner show the individual datapoints. d) Violin plots of eigengene expression of M4, M5 and M6 in inflamed tissues of IBD patients with $(n=61)$ or without $(n=111)$ deep ulceration observed in a replication cohort of paediatric CD and UC; horizontal lines indicate median and adjusted two-tailed Wilcoxon signed rank test P-values are given; exact P-values are $1.4 \times 10^{-4}$ and $1.1 \times 10^{-6}$ for M4 and M5 comparisons, respectively. e) Classification of M4/M5 high and M4/M5 low patients in the paediatric replication cohort $(n=172)$, based on hierarchical clustering on module eigengene values. f) Percent ulceration on biopsies collected within 3 months before anti-TNF therapy start (subcohort of Extended Data Fig. 3), as semiquantitatively (0-25-50-75-100) scored by a blinded consultant histopathologist ( $n=12$ responders, $n=10$ non responders). Two-tailed Mann-Whitney $U$ test $\mathrm{P}$-values are given. Therapy response was defined as described in Extended Data Fig. 3. 
a

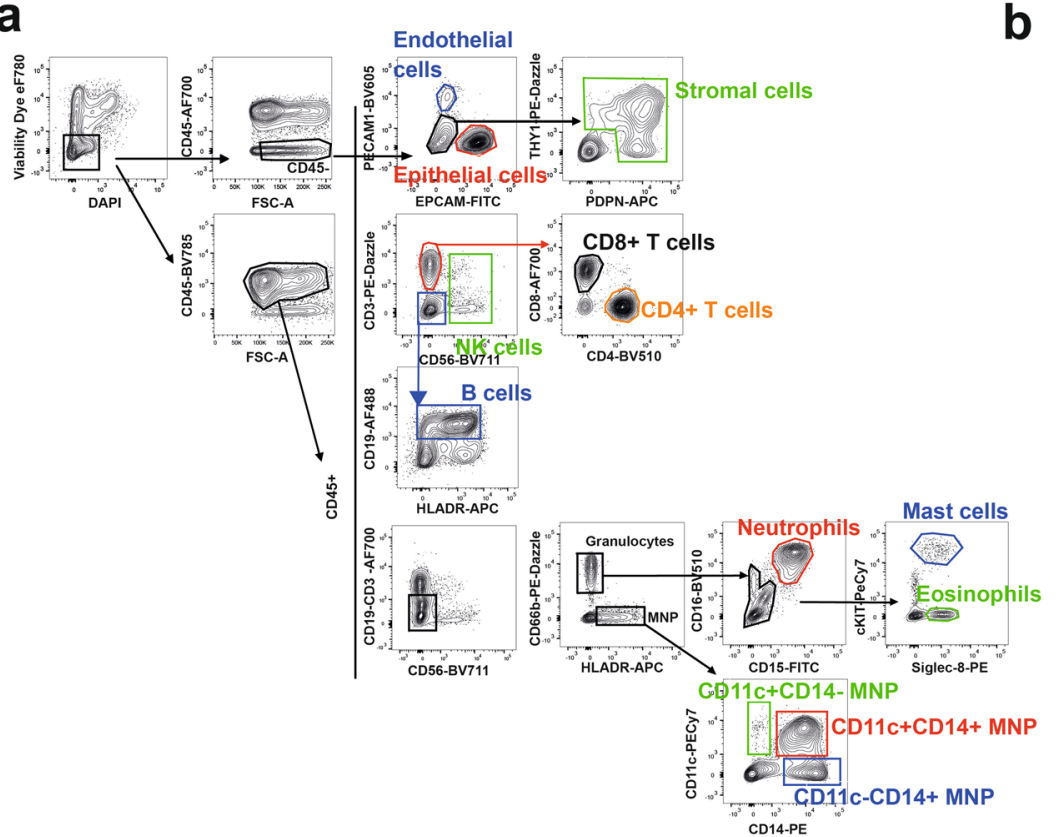

b

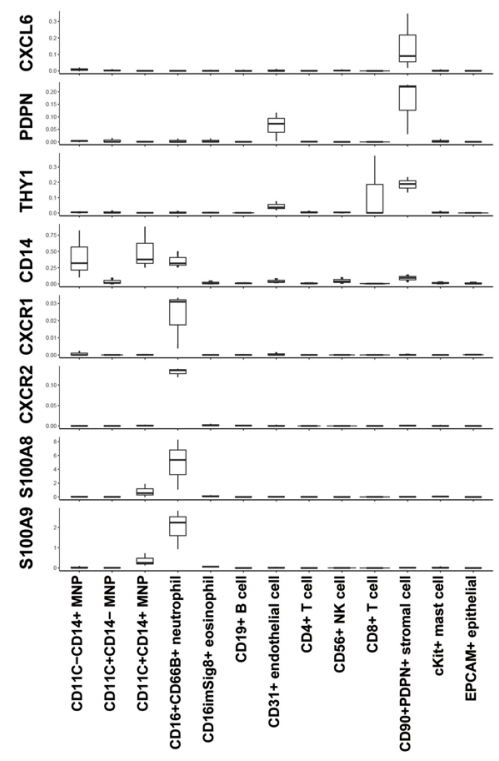

C

e
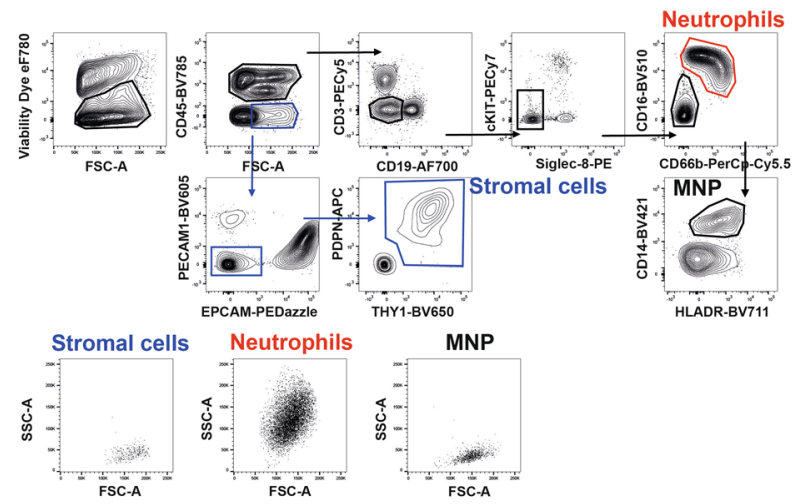

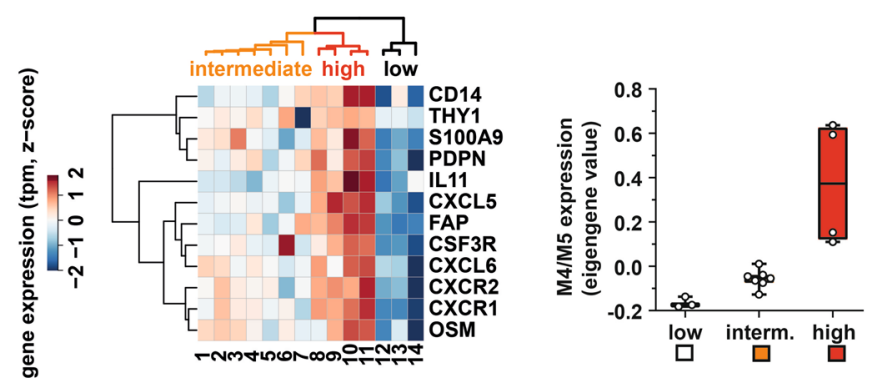

f

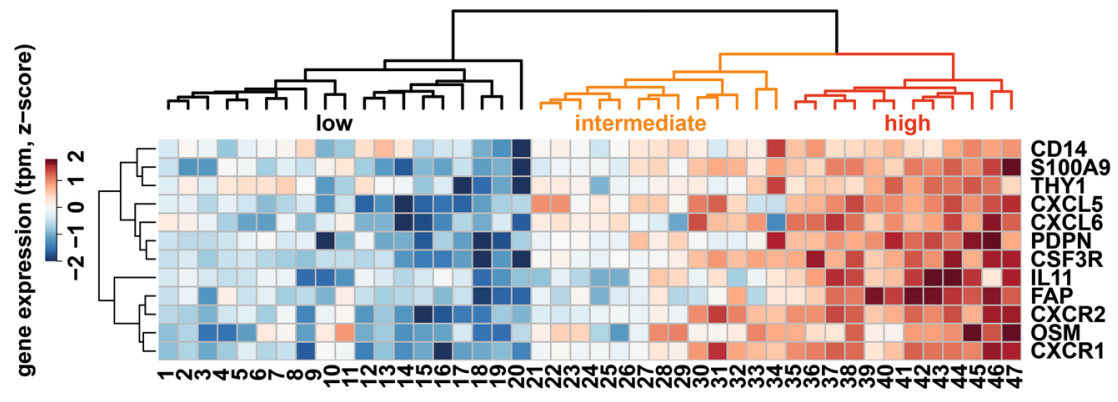

d
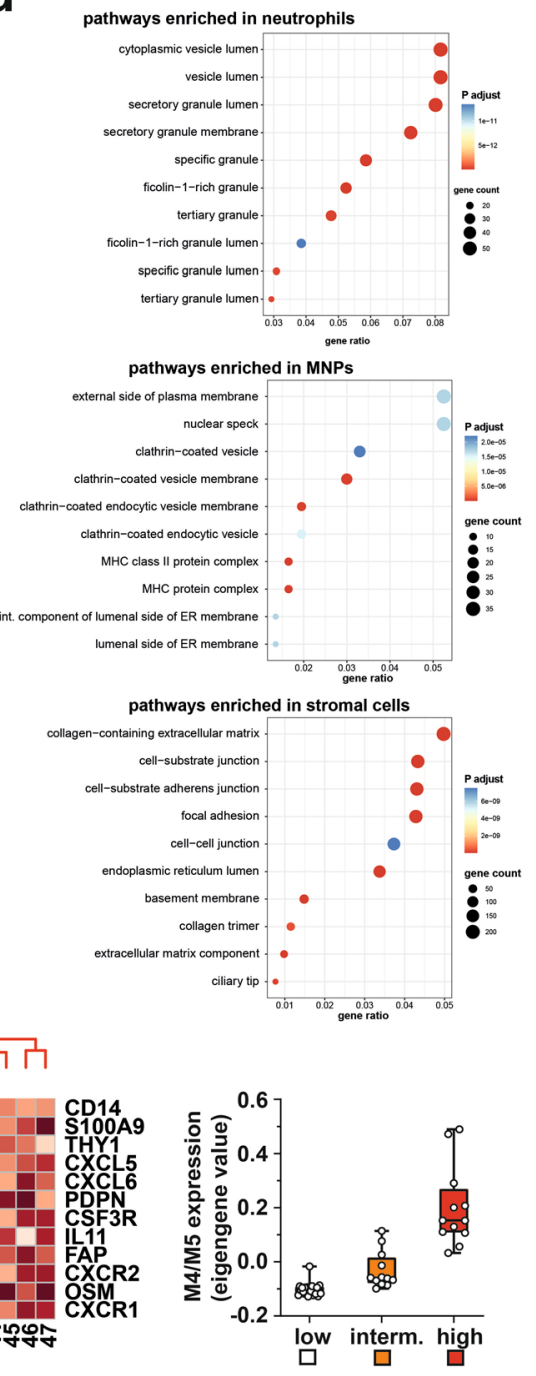

Extended Data Fig. 5 | See next page for caption. 
Extended Data Fig. 5 | FACS sorting and transcriptomic analysis of cellular subsets. a) Representative gating strategy of FACS sorting hematopoietic and non-hematopoietic cell populations from non-IBD and IBD patient tissue. b) Normalised gene expression (qPCR, relative to RPLPO expression) of selected genes from M4 and M5 in cell populations sorted as in a) from $n=3$ intestinal tissues of $n=3$ patient with IBD; box and whisker plots display median, upper and lower quartiles, and range. c) FACS-gating strategy for sorting of neutrophils, stromal cells and MNPs from tissue samples of IBD patients. d) Gene set enrichment analysis using Gene Ontology (GO) Cellular Components pathway terms, based on all genes significantly enriched ( $p$ adjusted $<0.05, \mid \log 2$ fold change $\mid>2$; two-tailed randomisation test) in either neutrophils MNPs or stromal cells (Supplementary Table 5); Exact $\mathrm{P}$-values can be obtained when re-running the analysis on https://github.com/microbialman/IBDTherapyResponsePaper.e $+f$ ) Heatmaps of whole tissue gene expression of selected genes that are representative (=highly correlative) of M4 and M5 expression (qPCR, z-score transformed gene expression values); unsupervised clustering (Manhattan) distinguishes subgrouping into M4/M5 low, intermediate and high samples; the box-plots on the right show the eigenvalues of all detected genes on a per patient basis; box and whisker plots display median, upper and lower quartiles, and range. The respective heatmaps refer to tissue samples used for FACS analysis $(n=14, e)$ and IHC analysis $(n=47, f)$ as shown in Fig. $3 d$, $f$ and $g$. 


\begin{tabular}{|c|c|c|}
\hline \multicolumn{3}{|c|}{ Bulk RNAseq and FACS analysis (Figure 3) } \\
\hline Characteristic & $\begin{array}{l}U C \\
N=9\end{array}$ & $\begin{array}{l}\mathrm{CD} \\
\mathrm{N}=3\end{array}$ \\
\hline$\%$ Male (Male/Female) & $33.3(3 / 9)$ & $100(3 / 3)$ \\
\hline $\begin{array}{l}\text { Median (IQR) age at } \\
\text { sampling (years) }\end{array}$ & $39(20-72)$ & $29(22-50)$ \\
\hline $\begin{array}{l}\text { Median (IQR) disease } \\
\text { duration at sampling (years) }\end{array}$ & $2(0-25)$ & $15(5-34)$ \\
\hline \multicolumn{3}{|l|}{ Nancy histologic score (\%) } \\
\hline \multirow{5}{*}{$\begin{array}{l}=0 \\
=1 \\
=2 \\
=3 \\
=4\end{array}$} & $0(0)$ & \\
\hline & $0(0)$ & $\mathrm{n} / \mathrm{a}$ \\
\hline & $3(33.3)$ & \\
\hline & $4(44.4)$ & \\
\hline & $2(22.2)$ & \\
\hline \multicolumn{3}{|c|}{ single-cell RNAseq (Figures 4 and 5) } \\
\hline Characteristic & $U C=7$ & Healthy $=4$ \\
\hline$\%$ Female & $100 \%$ & $100 \%$ \\
\hline $\begin{array}{l}\text { Median (IQR) age at } \\
\text { sampling (years) }\end{array}$ & $56(37-82)$ & $38(21-73)$ \\
\hline $\begin{array}{c}\text { Median (IQR) disease } \\
\text { duration at sampling (years) }\end{array}$ & $17(4-62)$ & $\mathrm{n} / \mathrm{a}$ \\
\hline \multirow[t]{6}{*}{ Nancy histologic score (\%) } & & $\mathrm{n} / \mathrm{a}$ \\
\hline & & \\
\hline & $1(14.28)$ & \\
\hline & & \\
\hline & $2(28.57)$ & \\
\hline & $4(57.1)$ & \\
\hline
\end{tabular}

Extended Data Fig. 6 | Clinical characteristics of the IBD patients used for RNAseq and FACS analysis. Clinical characteristics of the IBD patient cohorts used for the transcriptomic and FACS analysis. UC, Ulcerative colitis; IQR, interquartile range; UCEIS, Ulcerative Colitis Endoscopic Index of Severity. 
a

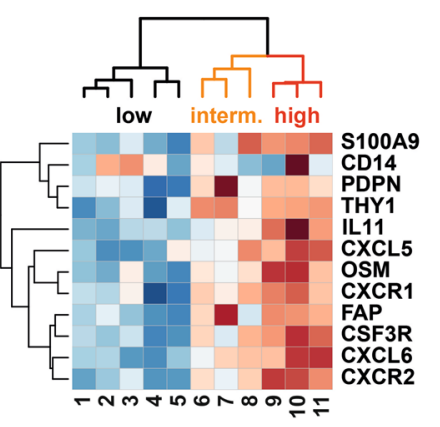

b

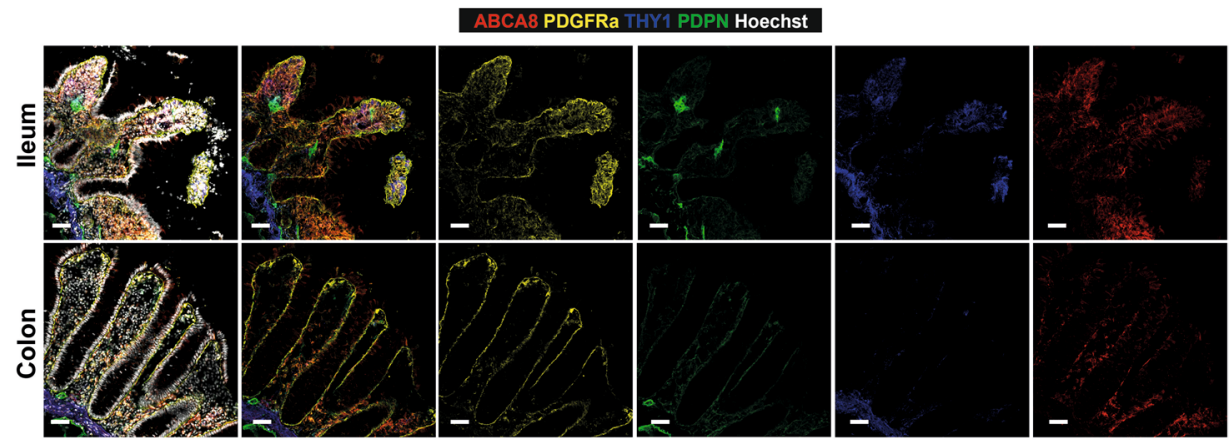

C

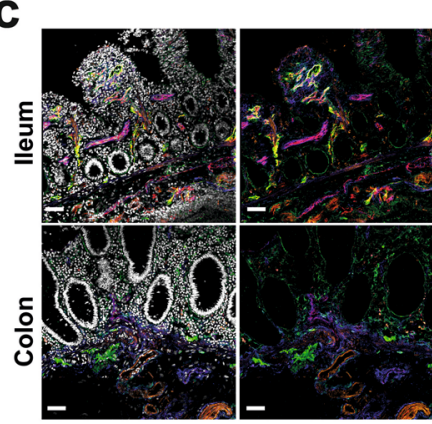

PECAM1 MCAM THY1 PDPN Hoechst
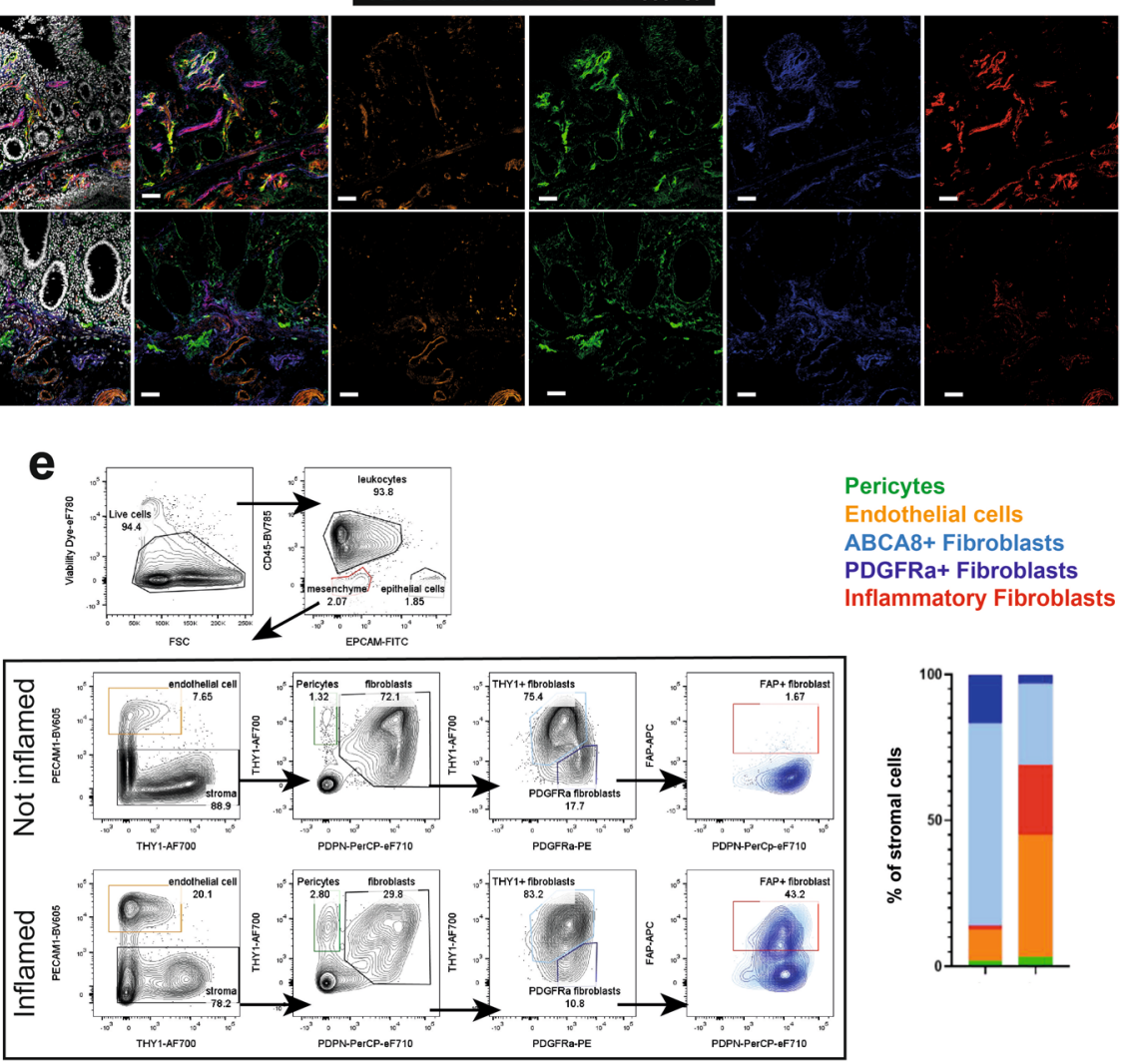

e

d

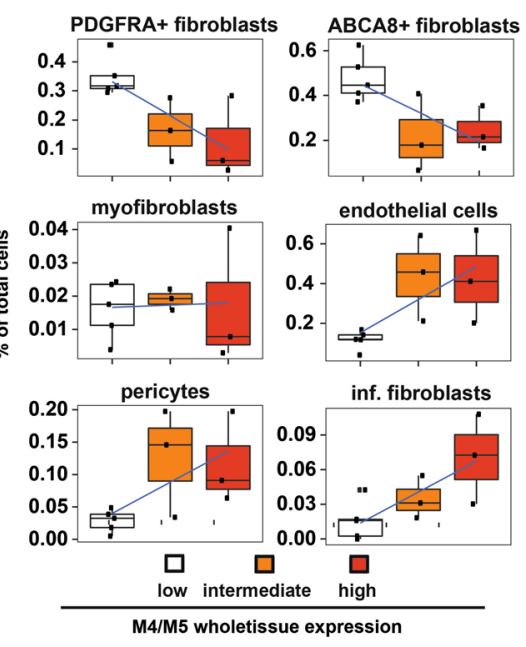

f
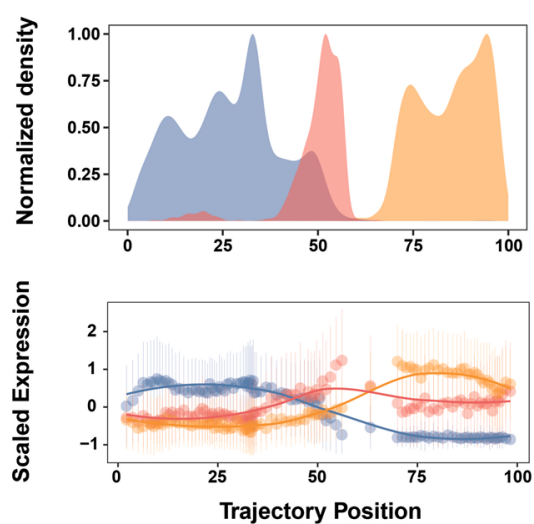

g

ABCA8+ Fibroblasts PDGFRa+ Fibroblasts Inflammatory Fibroblasts

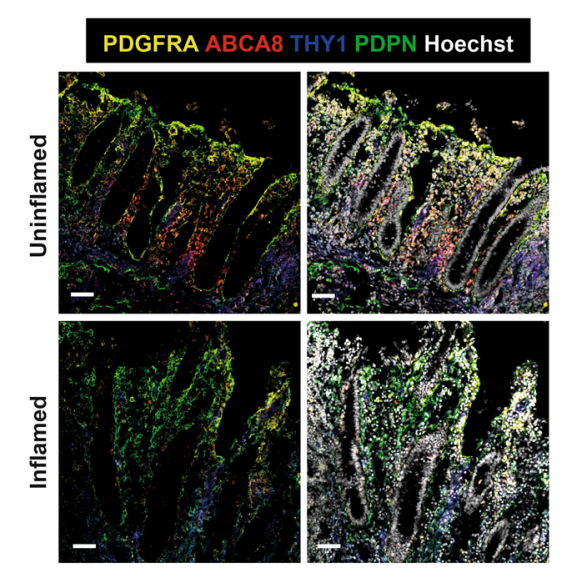

Extended Data Fig. 7 | See next page for caption. 
Extended Data Fig. 7 | Single-cell transcriptomic and immunofluorescent profiling of patient samples. a) Heatmap of whole tissue gene expression of selected genes that are representative of (highly correlated with) M4 and M5 expression (qPCR, $z$-score transformed gene expression values); unsupervised clustering (Manhattan) groups samples into M4/M5 low $(n=5$ patients), intermediate $(n=3$ patients) and high ( $n=3$ patients) from the set of IBD patients whose samples were profiled by single cell RNA sequencing; the box-plots (lower panel) show the eigenvalues of all detected genes on a per patient basis; data are presented as median values +/- range. b) Immunofluorescent staining of ABCA8 (red), PDGFRA (yellow), THY1 (blue), Podoplanin (PDPN, green) and nuclei (Hoechst, grey) in ileum and colon of resected tissue from IBD patients (not inflamed) (Images representative of stainings in $n=8$ tissues of $n=4$ patients). c) Immunostaining of PECAM1 (red), MCAM (orange) THY1 (blue), Podoplanin (PDPN, green) and nuclei (Hoechst, grey) in ileum and colonic resected tissue from IBD patients (not inflamed) (Images representative of stainings in $n=8$ tissues of $n=4$ patients). d) Box plot showing the proportion of the cell types in M4/M5 low ( $n=5$ patients), intermediate $(n=3$ patients $)$ and high groups ( $n=3$ patients), as detected by scRNAseq; box and whisker plots display median, upper and lower quartiles, and range. e) FACS analysis of live stromal cells (CD45-, EPCAM-) in resected tissue from an IBD patient (adjacent not inflamed and inflamed tissue). Gates for endothelial cells (PECAM1+), pericytes ( THY +, PDPN-), ABCA8 + fibroblasts (THY1 high, PDGFRa low), PDGFRA + fibroblasts (PDGFRA high, THY1 low) and inflammatory fibroblasts (FAP+) are shown. f) Pseudotime analysis of ABCA8 +, PDGFRA + and inflammatory fibroblasts in the single-cell dataset. Cell densities (top row) or canonical markers (bottom) are shown along the trajectory, binned to 100 uniform-density windows (each window has the same number of cells). $g$ ) Representative immunofluorescent stainings of PDGFRA (yellow) and ABCA8 (red) staining on fibroblasts in paired inflamed and uninflamed samples of the same IBD patient (Images representative of stainings in $n=8$ tissues of $n=4$ patients). 
a (relative to RPLP0)

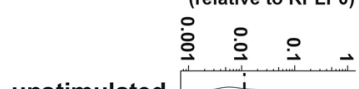

unstimulated

IL1- $\beta$

TNF-a

IL-6 : :

IL-11

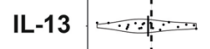

IL-17A $\cdots+. .$.

OSM $\cdots$

TGF- $\beta 1$ :

IFN-Y

LPS …….
CXCL5 expression

b

gene expression (log2, z-score) $-2 \begin{array}{llll}-1 & 0 & 1 & 2\end{array}$

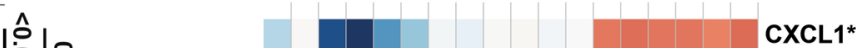
|

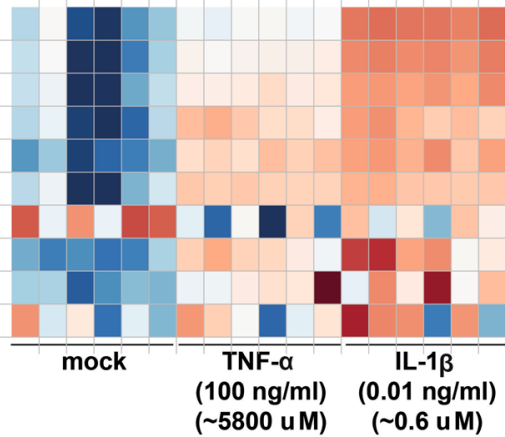

CXCL1*

$\mathrm{CXCL3}^{*}$

$\mathrm{CXCL5}^{*}$

CXCL6*

CXCL8*

THY1

PDPN $^{*}$

FAP*

OSMR

$\begin{array}{cc}(100 \mathrm{ng} / \mathrm{ml}) & (0.01 \mathrm{ng} / \mathrm{ml}) \\ (\sim 5800 \mathrm{u} \mathrm{M}) & (\sim 0.6 \mathrm{u} \mathrm{M})\end{array}$

d

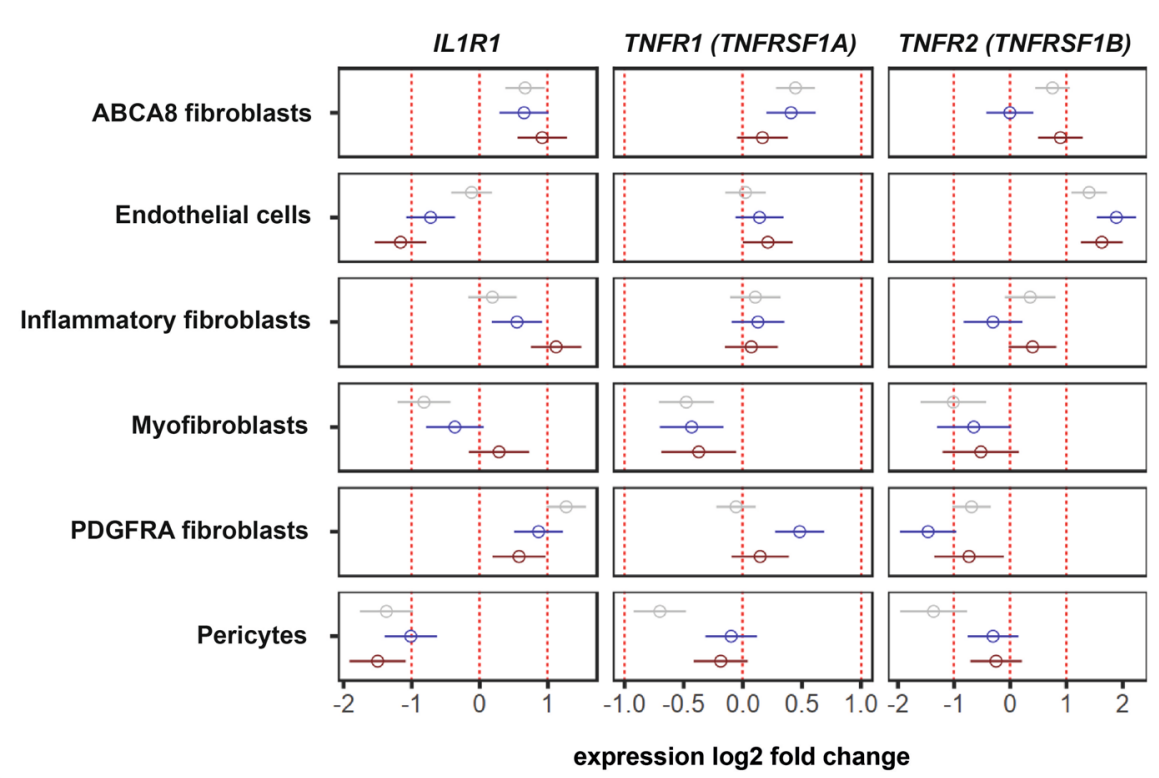

e

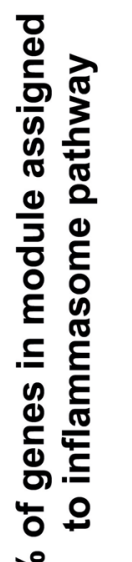

of

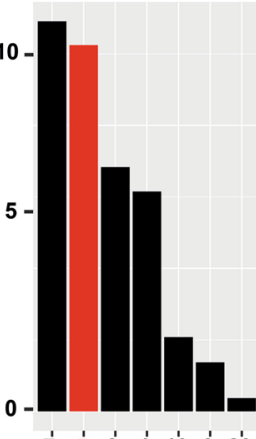
module
C

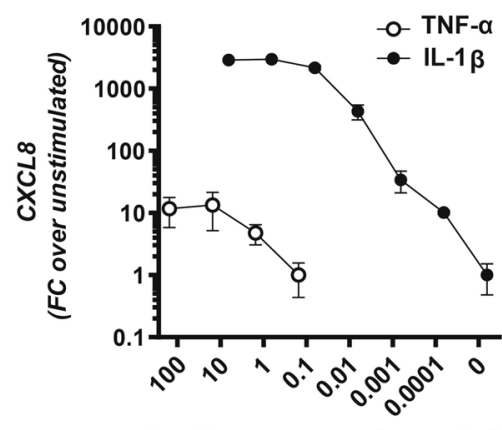

Cytokine concentration (ng/ml)
M4/M5 expression:

o high

o intermediate

○ low

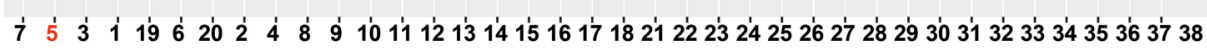

Extended Data Fig. 8 | See next page for caption. 
Extended Data Fig. 8 | Cytokine response of intestinal fibroblasts. a) Primary fibroblast cell lines $(n=33)$ culture-expanded from resected IBD patient tissue and stimulated for $3 \mathrm{~h}$ with recombinant cytokines (adjusted P-values are shown where significantly different $(p<0.05)$ compared to unstimulated, Kruskall-Wallis test). b) RNAseq analysis (Salmon log2-transformed TPM values, z-score) of cultured intestinal fibroblast cell line Ccd18-co, stimulated with either TNF- $\alpha(100 \mathrm{ng} / \mathrm{ml})$ or IL-1 $\beta(0.01 \mathrm{ng} \cdot \mathrm{ml})$ for 3 hours $\left(n=6\right.$ replicates per group, ${ }^{\star} \mathrm{P}$ adjusted $<0.05$ from DESeq2 differential gene expression analysis). c) Dose response of IL-1 $\beta$ and TNF- $\alpha$ stimulated Ccd18co fibroblasts for gene expression fold change (FC) of CXCL8 over unstimulated, measured by qPCR; data are presented as mean values $(n=2)+/-$ SD. $d$ ) Pseudo-bulk expression fold changes (relative to M4/M5 low groups) of ILR1 and TNFR1 (see Supplementary Table 7) within the cellular clusters detected as in Fig. 3a, across patients with either low, intermediate or high M4/M5 whole tissue expression. e) Gene set enrichment analysis of all modules detected in the discovery cohort for genes assigned to inflammasome pathways (GO:0061702). 


\section{Reporting Summary}

Nature Research wishes to improve the reproducibility of the work that we publish. This form provides structure for consistency and transparency in reporting. For further information on Nature Research policies, see our Editorial Policies and the Editorial Policy Checklist.

\section{Statistics}

For all statistical analyses, confirm that the following items are present in the figure legend, table legend, main text, or Methods section.

$\mathrm{n} / \mathrm{a}$ Confirmed

$\bigotimes$ The exact sample size $(n)$ for each experimental group/condition, given as a discrete number and unit of measurement

\ A statement on whether measurements were taken from distinct samples or whether the same sample was measured repeatedly

The statistical test(s) used AND whether they are one- or two-sided

Only common tests should be described solely by name; describe more complex techniques in the Methods section.

\A description of all covariates tested

$\square$ \ A description of any assumptions or corrections, such as tests of normality and adjustment for multiple comparisons

$\square$ A full description of the statistical parameters including central tendency (e.g. means) or other basic estimates (e.g. regression coefficient)

AND variation (e.g. standard deviation) or associated estimates of uncertainty (e.g. confidence intervals)

$\square$ For null hypothesis testing, the test statistic (e.g. $F, t, r$ ) with confidence intervals, effect sizes, degrees of freedom and $P$ value noted

For null hypothesis testing, the test statistic
Give $P$ values as exact values whenever suitable.

Х $\square$ For Bayesian analysis, information on the choice of priors and Markov chain Monte Carlo settings

Х $\square$ For hierarchical and complex designs, identification of the appropriate level for tests and full reporting of outcomes

$\square \bigotimes$ Estimates of effect sizes (e.g. Cohen's $d$, Pearson's $r$ ), indicating how they were calculated

\section{Our web collection on statistics for biologists contains articles on many of the points above.}

\section{Software and code}

Policy information about availability of computer code

Data collection Bulk RNA sequencing : Illumina HiSeq4000; Single Cell RNAsequencing : Illumina NextSeq 500; Image acquisition : NanoZoomer S210 Hamatsu (NDP.view2 U12388-21) Zeiss Confocal LSM 880 (Zen System 3.4); FACS acquisition and sort : LSRII, Aria III (FACSDiva v8.0); quantitative PCR: ViiA 7 Real-Time PCR System (QuantStudio 6)

Data analysis Bulk RNA sequencing data were analysed in Python using the bulk processing aspect of pipeline_scrnaseq.py (https://github.com/sansomlab/ scseq). Data quality was assessed using pipeline_readqc.py (https://github.com/cgat-developers/cgat-flow). Sequence read were aligned using the hisat2_extract_splice_sites.py tool. Mapped Reads were counted using featureCounts (Subread version 1.6.3). Salmon v0.9.1 was used to calculate TPM values. $\mathrm{R}$ (v3.6.1) packages_version used for analysis were as follows:

rstatix_0.7.0; clusterProfiler_3.14.3; gmodels_2.18.1; simpleaffy_2.62.0; gcrma_2.58.0; genefilter_1.68.0; affy_1.64.0; knitr_1.33;

biomaRt_2.42.1; annotate_1.64.0;XML_3.99-0.3;BiocManager_1.30.15; stringi_1.6.2;egg_0.4.5; gridExtra_2.3; gtools_3.8.2; ggrepel_0.9.1; GO.db_3.10.0; org.Hs.eg.db_3.10.0; AnnotationDbi_1.48.0; IRanges_2.20.2;S4Vectors_0.24.4;vroom_1.4.0;

RColorBrewer_1.1-2;gplots_3.1.1; edgeR_3.28.1;limma_3.42.2;pROC_1.17.0.1;data.table_1.14.0;OptimalCutpoints_1.1-4;caret_6.0-88 ;lattic e_0.20-38;ROCR_1.0-11;ROCit_2.1.1;ggpubr_0.4.0;WGCNA_1.70-3;fastcluster_1.1.25; dynamicTreeCut_1.63-

Z;forcats_0.5.1;stringr_1.4.0; dplyr_1.0.6;purr__0.3.4;readr_1.4.0;tidyr_1.1.3;tibble_3.1.2;ggplot2_3.3.3;tidyverse_1.3.1;GEOquery_2.54.1;Bio base_2.46.0;BiocGenerics_0.32.0; gplots_3.1.1,DESeq2_1.26.0;xCell_1.1.

Microarray from publicly available sets were processed and normalised using the affy package (v1.64.0). Meta-analysis of the expression of modules in the replication datasets was carried out using the meta package (v4.19.0) and AUC values for each gene were generated by applying the roc function from the pROC (v1.17.0.1) package in R.

Quantitative histopathology were carried out in Indica Labs $\mathrm{HALO}^{\circledast}$ image analysis platform using Indica Labs analysis modules CytoNuclear v2.0.5 and v2.0.9. Fluorescent image were analyzed and merged using imageJ software (v1.53c).

Single-cell sequencing data was processed using Cell Ranger (v3.1.0), Kallisto (v0.46.0) and Bustools (https://github.com/BUStools/bustools) (v0.39.0). PCA analysis was performed with the VST method implemented in the Seurat R package and R Spectra (https://github.com/yixuan/ RSpectra). Batch effects in the PCA embeddings, was modelled and removed using the Harmony algorithm. Harmonized UMAP was generated with the UMAP (arXiv:1802:03426 [stat.ML]) algorithm. Data analysis was then performed using Seurat and Deseq2 and princurve R (https:// 
www.jstor.org/stable/2289936) packages in R software.

FACS analysis was performed using FlowJo v(10.7.1).

Figure visualisation and statistics analysis were performed using GraphPad v8.4.2, Adobe illustrator 2019 (v23.1) and R (v3.61 and 4.0.1).

All details and code relating to these analyses is available in the following Github repository: https://github.com/microbialman/

IBDTherapyResponsePaper

For manuscripts utilizing custom algorithms or software that are central to the research but not yet described in published literature, software must be made available to editors and

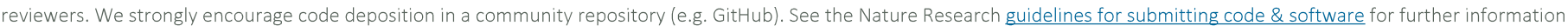

\section{Data}

Policy information about availability of data

All manuscripts must include a data availability statement. This statement should provide the following information, where applicable:

- Accession codes, unique identifiers, or web links for publicly available datasets

- A list of figures that have associated raw data

- A description of any restrictions on data availability

Bulk RNA sequencing has been deposited on GEO (GSE166928) and single-cell data on ImmPort (SDY1765; accessible with the next release scheduled for September 10 2021). Additional data has been made available through a GitHub repository at https://github.com/microbialman/IBDTherapyResponsePaper. Due to the extensive size, image scans, and raw data for FACS/qPCR assays are available from the corresponding author (FMP) upon request.

Publicly available RNAseq (GSE57945, GSE109142) or microarray data (GSE16879, GSE12251, GSE100833) were downloaded from the NCBI gene expression omnibus.

\section{Field-specific reporting}

Please select the one below that is the best fit for your research. If you are not sure, read the appropriate sections before making your selection.

$\bigotimes$ Life sciences $\quad \square$ Behavioural \& social sciences $\quad \square$ Ecological, evolutionary \& environmental sciences

For a reference copy of the document with all sections, see nature.com/documents/nr-reporting-summary-flat.pdf

\section{Life sciences study design}

All studies must disclose on these points even when the disclosure is negative.

Sample size $\quad$ The number of available samples was dictated by the number of interventions (surgeries, endoscopies) carried out, of which we collected every possible sample. Conclusions in the manuscript relating to this data are supported by appropriate statistical tests, and where possible all datapoints are shown.

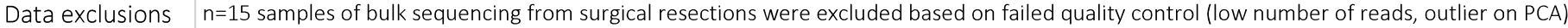
(see GSE166928 for flags and https://github.com/microbialman/IBDTherapyResponsePaper)

Replication

Given the sample nature, technical replication of experiments involving human patient sample material was not carried out as collecting substantially more tissue could not be ethically supported. Patient data was analysed at a cohort level, using all patients/samples/datapoints, to derive statistically meaningful conclusions. For experiments with cell lines, replicates are presented and experiments only shown if the experiment could was successfully repeated at least one other occasion.

Randomization Randomization was not relevant to the clinical findings as this was an observational cohort. Cell culture wells were randomly allocated to experimental groups.

Blinding All human samples and cells lines used in this paper were blinded before data collection by giving them a unique ID number. Pathologists were blinded for scoring of histopathologic slides.

\section{Reporting for specific materials, systems and methods}

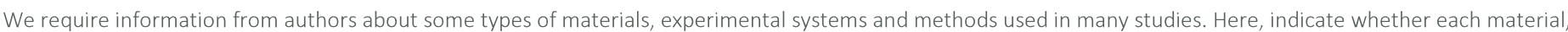

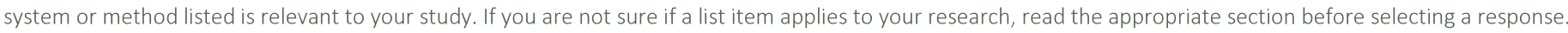




\begin{tabular}{l|l}
\hline$n / a$ & Involved in the study \\
\hline & $\bigotimes$ Antibodies \\
$\square$ & $\bigotimes$ Eukaryotic cell lines \\
$\square$ & $\square$ Palaeontology and archaeology \\
$\square$ & $\square$ Human research participants \\
$\square$ & $\square$ Clinical data \\
& $\square$ Dual use research of concern
\end{tabular}

\section{Antibodies}

Antibodies used

Antibodies for FACS and IF analysis or sorting cells were from Biolegend (all used @1:200 dilution except for CD45, CD56, CD3, PDGFRA, CD19 @1:100, and CD4, CD8, Sig8 @1:50): BV785 antiCD45 (clone HI30 ref 304024, lot B284678), AF700 antiCD45 (clone HI30, ref 304048, lot B219921), APC antiHLADR (clone L243, ref 307610, lot B214996), BV711 antiHLADR (clone L243, ref 307644, lot B271297), PE antiCD14 (clone HCD14, lot B269397), BV421 antiCD14 (clone HCD14, ref 325628, lot B286544), PeCy5 antiCD3 (clone UCHT1, ref 300410, lot B258253), AF700 antiCD3 (clone SK7, ref 344828, lot B245810), Pedazzle antiCD3 (clone SK7, ref 344844, lot B220096), PEdazzle antiCD66b (clone G10F5, ref 305122, lot B279539), PerCpCy5.5 antiCD66b (clone G10F5, ref 305108, lot B260775), BV711 antiCD56 (clone 5.1h11, ref 362542, lot B327219), AF488 antiCD19 (clone HIB19, ref 302219, lot B238185), AF700 antiCD19 (clone HIB19, ref 30226, lot B254252), Pecy7 antiCD11c (clone S-HCL-3, ref 371508, lot B258655), BV510 antiCD16 (clone 3G8, ref 302048, lot B265264), PE antiSiglec8 (clone 7C9, ref 347104, lot B261537), Pedazzle antiCD15 (clone W6D3, ref 323038, lot B228060), PeCy7 antiCKIT (clone 104D2, ref 313212, lot B264778), BV510 antiCD4 (clone OKT4, ref 317444, lot B252921), AF700 antiCD8A (clone SK1, ref 344724, lot B282429), BV605 antiPECAM1 (clone WM59, ref 303122, lot B229211), AF647 antiPECAM1 (clone WM59, ref 303112, lot B161860), FITC antiEPCAM (clone 9C4, ref 324204, lot B261790), Pedazzle antiEPCAM (clone 9C4, ref 324232, lot B270029), Pedazzle antiTHY1 (clone 5E10, ref 328134, lot B266076), BF650 antiTHY1 (clone 5E10, ref 328144, lot B263262), PE antiPDGFRa (clone 16A1, ref 323506, lot B275570). eBioscience : PerCp-eFluor 710 antiPDPN (clone Nz1.3, ref 46-9381-42, lot 4332205) , APC antiPDPN (clone Nz1.3, ref 17-9381-42, lot 4341948). R\&Dsystems : APC antiFAP (Clone 427819, ref FAB3715A-100, lot AEHI0119011).

Further antibodies used for IHC/IF studies were: AF488 antiNeutrophil Elastase @1ug/ml (clone NP57, company :Santacruz, ref sc-53388-AF488, lot G1019), antiPDPN @1:100 (clone D2-40, company :Dako, ref ISO72, lot 10095921), antiPDPN @5ug/ml (clone Nz1.3, company ; eBioscience, ref 17-9381-42, lot 2065602), antiTHY1 @5ug/ml (clone 5E10, biolegend, ref 328102, lot B216386), antiNeutrophil Elastase @1:100 (clone NP57, company Santacruz, ref sc-53388, lot : G0810), AF488 antiMCAM @5ug/ml (clone P1H12, company Biolegend, ref 361020, lot B270164), Biotinylated antiFAP @1:100 (polyclonal, company : R\&D systems, ref AF3715), antiCD68 @1:500 (polyclonal, company : Sigma-Aldrich, ref HPA048982, lot 007002291), antiCalprotectin @ 1:100 (clone MAC387, company :Bio-Rad, ref MCA874G, lot : 1801), antill1b @1:100 (clone 3A6, company : Cellsignalling, ref : 12242S, lot :1), antiABCA8 1ug/ml (polyclonal, company : ThermoFisher, ref PA5-60866, lot A105833), antiPDGFRa @5ug/ml (polyclonal, company : R\&Dsystems, ref AF-307-NA, lot VG0718081). Secondary antibodies included : Biotinylated anti-rabbit IgG @1:500 (company : Vectorlab, ref BA-1000, lot ZE1218), Biotinylated anti-mouse @1:500 (company : Vector lab, ref BA-2000, lot ZG0430), AF488 antiRat IgG @1:500 (company : Invitrogen, ref A-31572, lot 2017396), AF647 antiGoat @1:500 (company : invitrogen, ref A-21447, lot 1739289).

Isotype control included : AF647 mouselgG1 (clone MOPC-21, company Biolegend, ref 400135, lot B241547), AF488 mouse lgG1 (clone MOPC-21, company :Biolegend, ref : 400129, lot B27964), AF488 mouselgG1 (company: santacruz, ref sc-3890, lot K2619), Rat IgG2a (company :ebioscience, ref 16-4321-82, lot 2269842), mouse IgG1 (Company : SigmaAaldrich, ref 02-6100, lot TB266463)

Validation

FACS :

All antibobies from Biolegend have been validated by the manufacturer for use in flow cytometry. Additional information can be obtained on the company websites.

anti-PDPN, clone Nz1.3 Percp eFluor710 /APC have been validated by the manufacturer for flow cytometry. antiFAP APC antibody has been validated by the manufacturer for flow cytometry. IHC:

Mouse anti S100A9 (Clone MAC387- Biorad), Rabbit polyclonal antiCD68 (Reference HPA048982 Sigma-aldrich), Mouse anti human NE (NP47- SantaCruz), Goat anti PDGFRA (Reference : AF-307-NA Biotechne), IL1beta (Clone 3A6 CellSignalling 12242S), mouse antiPDPN antibody (Clone D2-40; Dako) were validated by manufacturer for use in IHC on paraffin.

Biotinylated sheep anti-FAP (Reference : AF3715, Biotechne) was validated in house for IHC on paraffin sections, normal adjacent CRC tissue was used as a negative control for antibody specificity.

IF:

Rat anti human PDPN (NZ 1.13- Ebioscience), biotinylated sheep anti-FAP (Reference : AF3715, Biotechne), Goat anti PDGFRA (Reference : AF-307-NA Biotechne), Rabbit anti ABCA8 (PA5-60866, ThermoFisher). -Conjugated, Mouse anti human THY1 (5E10, Biolegend), AF488 anti NE (NP57, Santa cruz), AF647 anti PECAM1 (WM59, Biolegend), AF488 mouse antiMCAM (P1H12, Biolegend) were all validated in house for use in IF after PLP fixation. Unspecific binding was assessed with Isotype control.

\section{Eukaryotic cell lines}

Policy information about cell lines

Cell line source(s)

Ccd18-Co ( ATCC, ref : CRL-1459) colonic fibroblast cell lines

Authentication

STR profiling by the vendor 
Commonly misidentified lines (See ICLAC register)

\section{Human research participants}

\section{Policy information about studies involving human research participants}

Population characteristics

We analyzed 39 non IBD surgical resection from large and small intestine (54\% Male, age median $68,39 \%$ of large intestine), 31 IBD surgical resection from large and small intestine (25.8\% UC, 70.9\% CD, 3,22\% IBDu, 52\% Male, age median 37, 27\% large intestine). Patients medications before surgery was analyzed as covariates and included $19 \%$ of aminosalicylates, $39 \%$ of corticosteroids, $68 \%$ of immunomodulators, $42 \%$ of antiTNFa antibodies, $7 \%$ of anti-integrin antibodies.

We also analysed intestinal biopsies sections from UC patients under anti-TNFa therapy. 35 patients were responding to treatment ( $65,7 \%$ Male, age median 32), 23 patients were not responding to antiTNFa (53.3\% Male, age median 34). Additionally, untestinal biopsies from IBD patients were used for FACS and sorted bulk RNA sequencing analysis including 9 UC and 3 CD patients ( $50 \%$ Male, age median 34).

4 Healthy biopsies and 7 UC biopsies were used for single cell RNA sequencing ( $100 \%$ Female, age median 47 ).

Recruitment

Patients were recruited through the Oxford TGU Biobank, UK. Intestinal biopsies were taken from patients followed up at the John Radcliffe hospital for their IBD management; healthy intestinal biopsies were taken from patients undergoing endoscopy for suspected intestinal symptoms, but where IBD/intestinal inflammation was not diagnosed. Surgical resection were obtained from surgeries scheduled as part of the disease management for patients with IBD or CRC. Patients analysed for single-cell RNAseq were exclusively female; no gender bias in IBD is reported, hence it can be assumed that gender is not a confounding factor in any of our analyses.

Ethics oversight

All patients and healthy participants gave informed consent and collection was approved by NHS National Research Ethics Service under the research ethics committee references IBD 09/H1204/30 and 11/YH/0020 for IBD or GI 16/YH/0247 for CRC samples and gut biopsies from healthy individuals.

Note that full information on the approval of the study protocol must also be provided in the manuscript.

\section{Flow Cytometry}

Plots

Confirm that:

$\bigotimes$ The axis labels state the marker and fluorochrome used (e.g. CD4-FITC).

\The axis scales are clearly visible. Include numbers along axes only for bottom left plot of group (a 'group' is an analysis of identical markers).

\All plots are contour plots with outliers or pseudocolor plots.

$\bigotimes$ A numerical value for number of cells or percentage (with statistics) is provided.

\section{Methodology}

Sample preparation

Instrument

Software

Cell population abundance

Gating strategy
Minced tissue from intestinal biopsies or surgical resection was subjected to multiple rounds of digestion in RPMI1640 medium containing $5 \%$ fetal bovine serum (FBS), $5 \mathrm{mM} \mathrm{HEPES}$, antibiotics as above, and $1 \mathrm{mg} / \mathrm{ml}$ Collagenase A and DNase I (all from Sigma Aldrich). After 30 minutes, digestion supernatant containing cells was taken off, filtered through a cell strainer (50uM), spun down and resuspended in $10 \mathrm{ml}$ of PBS containing 5\% BSA and 5mM EDTA. Remaining tissue was then topped up with fresh digestion medium until no more cells were liberated from the tissue. Single-cell suspensions obtained from tissue digests were stained for FACS analysis or sorting with antibodies in PBS with 5\% BSA and 5mM EDTA for 20 minutes on ice. After washing in the same buffer, cells for FACS analysis were fixed for 20min in PFA4\% (BD fixative buffer) Sorted cells were not fixed but directly sorted in PBS with 5\% BSA and 5mM EDTA buffer.

FACS analysis was done on a LSRII (BD), cells were sorted on an Arialll sorter (BD)

FACS data were analyzed in FlowJo software v(10.7.1)

Between 15000 and 5000 Immune and stromal cell were sorted for Extended Figure 5b. Between 10000 and 100000 cells were sorted for Bulk RNA sequencing (Figure 3c). The number of cells depended on the inflammation status of the tissue and the size of the surgical pieces removed. Cell purity was confirmed by FACS analyzing fraction of the sorted cells, all cells sorted only expressed the selecting markers. Gene expression of the different marker on which cells were sorted was also confirmed by RTqPCR on sorted pure populations.

Cells were distinguished from debris based on FSC-SSC, single cells were isolated from doublets by FSC-H, FSC-W, live cells were distinguished from dead cells using negative selection for DAPI staining (Thermoscientific 62248) and Viability Dye EF780 (ebioscience, 65-0865-14).

Epithelial cells, Endothelial cells and Stromal cells were gated on CD45 negative event. EPCAM were then selected on EPCAM positivity, Endothelial cells on PECAM1 positivity. Stromal cells were identified as EPCAM, PECAM1 negative but THY1 and PDPN positive.

Leukocytes were gated on CD45 positivity. 
CD8 T cells were then gated on CD3e and CD8 positivity, CD4 T cells were gated on CD3e and CD4 positivity. NK cells were gated on CD56 positivity. B cells were gated on CD3 and CD56 negativity and CD19 positivity.

Granulocytes were gated on CD3, CD19, HLADR negativity and CD66b positivity. Neutrophils were gated on CD15 and CD16 positivity. Mast cells were gated on CD15 and CD16 negativity and CKIT positivity. Eosinophils were gated CD15 and CD16 intermediate positivity and SIGLEC8 positivity.

Myeloid cells were gated on CD3, CD19 and CD56, CD66b negativity and HLADR positivity. MNP were then distinguished based on CD14 and CD11c expression.

All cells were back gated on FSC and SSC to confirm size and granularity.

$\bigotimes$ Tick this box to confirm that a figure exemplifying the gating strategy is provided in the Supplementary Information. 